\title{
The BOSHPY Fluorophores: BODIPY Analogues with Single Atom Controlled Aggregation
}

\author{
Briana R. Schrage,$^{\dagger}$ Victor N. Nemykin, ${ }^{\S}$ and Christopher J. Ziegler ${ }^{* \dagger}$ \\ ${ }^{\dagger}$ Department of Chemistry, University of Akron, Akron, Ohio 44312-3601, United States \\ $\S$ Department of Chemistry, University of Tennessee, Knoxville, TN 37996, United States \\ *Corresponding author. Department of Chemistry, University of Akron, OH 44325-3601, USA \\ E-mail: ziegler@uakron.edu for the author(s) to whom correspondence should be addressed.
}

\section{Supplementary Information}




\section{Table of Contents}

General Information $\quad$ S5-6

$\begin{array}{ll}\text { Full citation for Gaussian } & \text { S7 }\end{array}$

$\begin{array}{ll}\text { Syntheses } & \text { S8-11 }\end{array}$

Figure S1: ${ }^{1} \mathrm{H}$ NMR (300 MHz) of 2 in d6-DMSO. $\quad$ S12

Figure S2: ${ }^{1} \mathrm{H}$ NMR (300 MHz) of $\mathbf{3}$ in d6-DMSO. $\quad$ S13

Figure S3: ${ }^{1} \mathrm{H}$ NMR $(300 \mathrm{MHz})$ of 4 in d6-DMSO. $\quad$ S14

Figure S4: ${ }^{1} \mathrm{H}$ NMR (300 MHz) of $\mathbf{1 B F} 2$ in d6-DMSO. $\quad$ S15

Figure S5: ${ }^{1} \mathrm{H}$ NMR (300 MHz) of 2BF2 in d6-DMSO. S16

Figure S6: ${ }^{1} \mathrm{H}$ NMR $(300 \mathrm{MHz})$ of $\mathbf{3 B F}_{\mathbf{2}}$ in d6-DMSO. $\quad$ S17

Figure S7: ${ }^{1} \mathrm{H}$ NMR $(300 \mathrm{MHz})$ of $\mathbf{4} \mathbf{B F}_{2}$ in d6-DMSO. $\quad$ S18

Figure S8: ${ }^{13} \mathrm{C}\left\{{ }^{1} \mathrm{H}\right\}$ NMR (125 MHz) of 2 in d6-DMSO. S19

Figure S9: ${ }^{13} \mathrm{C}\left\{{ }^{1} \mathrm{H}\right\}$ NMR $(125 \mathrm{MHz})$ of $\mathbf{3}$ in d6-DMSO. $\quad$ S20

Figure S10: ${ }^{13} \mathrm{C}\left\{{ }^{1} \mathrm{H}\right\}$ NMR $(125 \mathrm{MHz})$ of 4 in d6-DMSO. $\quad$ S21

Figure S11: ${ }^{13} \mathrm{C}\left\{{ }^{1} \mathrm{H}\right\}$ NMR $(125 \mathrm{MHz})$ of $\mathbf{1 B F} 2$ in d6-DMSO. $\quad$ S22

Figure S12: ${ }^{13} \mathrm{C}\left\{{ }^{1} \mathrm{H}\right\}$ NMR $(125 \mathrm{MHz})$ of $\mathbf{2 B F} 2$ in d6-DMSO. $\quad$ S23

Figure S13: ${ }^{13} \mathrm{C}\left\{{ }^{1} \mathrm{H}\right\}$ NMR $(125 \mathrm{MHz})$ of $\mathbf{3 B F} 2$ in d6-DMSO. $\quad$ S24

Figure S14: ${ }^{19} \mathrm{~F}$ NMR (282 MHz) of $\mathbf{1 B F}$ in d6-DMSO. S25

Figure S15: ${ }^{19} \mathrm{~F}$ NMR (282 MHz) of $\mathbf{2 B F}$ in d6-DMSO. S26

Figure S16: ${ }^{19}$ F NMR (282 MHz) of $\mathbf{3 B F}$ in d6-DMSO. $\quad$ S27

Figure S17: High-resolution ESI mass spectra of 2 S28

Figure S18: High-resolution ESI mass spectra of 3 S29

Figure S19: High-resolution ESI mass spectra of $4 . \quad$ S30

Figure S20: High-resolution ESI mass spectra of $\mathbf{1 B F}_{\mathbf{2}} \quad$ S31

Figure S21: High-resolution ESI mass spectra of $\mathbf{2 B F}$. $\quad$ S32

Figure S22: High-resolution ESI mass spectra of $\mathbf{3 B F}$. $\quad$ S33

Figure S23: High-resolution ESI mass spectra of $\mathbf{4 B F}$. $\quad$ S34

Figure S24: UV-visible spectra of 1-4 in DMF solution. $\quad$ S35

Figure S25: UV-visible spectra of $\mathbf{1 B F} \mathbf{-}-\mathbf{H F}_{\mathbf{2}}$ in DMF solution. $\quad$ S36

$\begin{array}{ll}\text { Figure S26: UV-Vis absorption titration with increasing amounts } & \text { S37 }\end{array}$ 
of $\mathbf{1 B F}_{2}$ in DMF (top), and normalized absorption spectra (bottom).

Figure S27: UV-Vis absorption titration with increasing amounts $\quad$ S38 of $\mathbf{2} \mathbf{B F}_{2}$ in DMF (top), and normalized absorption spectra (bottom).

Figure S28: UV-Vis absorption titration with increasing amounts $\quad$ S39 of $\mathbf{4 B F} 2$ in DMF (top), and normalized absorption spectra (bottom).

$\begin{array}{ll}\text { Figure S29: The normalized absorption (solid) and } & \text { S40 }\end{array}$ emission (dashed) spectra for compound $\mathbf{1} \mathbf{B F}_{\mathbf{2}}-\mathbf{4} \mathbf{B F}_{\mathbf{2}}$ in $\mathrm{DMF}$.

Figure S30: Cyclic voltammograms of $\mathbf{1 - 4}$ in $\mathrm{DMF}_{0.1} \mathrm{TBAPF}_{6} \quad \mathrm{~S} 41$

Figure S31: Cyclic voltammograms of $\mathbf{1 B F}_{\mathbf{2}}-\mathbf{4 B F} \mathbf{2}$ in DMF/0.1 TBAPF $6 \quad$ S42

Figure S32: DFT-predicted frontier orbitals for compounds 1-4. S43

Figure S33: DFT-predicted frontier orbitals for compounds $\mathbf{1 B F}_{\mathbf{2}}-\mathbf{4} \mathbf{B F}_{\mathbf{2}} \quad \mathrm{S} 44$

Figure S34: Experimental and B3LYP TDDFT-predicted $\quad$ S45 spectra for compounds 1-4.

Figure S35: Experimental and B3LYP TDDFT-predicted $\quad$ S46 spectra for compounds $\mathbf{1 B F}_{2}-\mathbf{4 B F} 2$.

$\begin{array}{ll}\text { Figure S36: B3LYP relative energies of the frontier } & \text { S47 }\end{array}$ orbitals for compounds 1-4.

Figure S37: B3LYP relative energies of the frontier $\quad$ S48 orbitals for compounds $\mathbf{1 B F} \mathbf{2}-\mathbf{4 B F} 2$.

Figure S38: Structure of compound 1, showing 35\% probability ellipsoids. $\quad$ S49

Figure S39: Structure of compound 2, showing 35\% probability ellipsoids. $\quad$ S50

Figure S40: Structure of compound 3, showing 35\% probability ellipsoids. S51

Figure S41: Structure of compound 4, showing 35\% probability ellipsoids. $\quad$ S52

Figure S42: Structure of compound $\mathbf{1 B F}$, showing 35\% probability ellipsoids. $\quad$ S53

Figure S43: Structure of compound $\mathbf{2} \mathbf{B F}$, showing 35\% probability ellipsoids. $\quad$ S54

Figure S44: Structure of compound $\mathbf{3 B F}$, showing 35\% probability ellipsoids. $\quad$ S55

Figure S45: Structure of compound $\mathbf{4 B F}$, showing 35\% probability ellipsoids. S56

Table S1: X-ray crystal data and structure parameters for compounds 1-4. S57

Table S2: X-ray crystal data and structure parameters for compounds $\mathbf{1 B F} \mathbf{2}-\mathbf{4 B F}$. $\quad$ S58

Table S3: Photophysical properties of compounds $\mathbf{1 B F}-\mathbf{4 B F}$ in DMF. S59

Table S4: B3LYP TDDFT-predicted energies and expansion coefficients for $\quad$ S60 compound 1 (only excited states with $\mathrm{f}>0.05$ and $\lambda>270 \mathrm{~nm}$ are listed).

Table S5: B3LYP TDDFT-predicted energies and expansion coefficients for $\quad$ S60 compound 2 (only excited states with $\mathrm{f}>0.05$ and $\lambda>270 \mathrm{~nm}$ are listed).

Table S6: B3LYP TDDFT-predicted energies and expansion coefficients for $\quad$ S60 
compound 3 (only excited states with $\mathrm{f}>0.05$ and $\lambda>270 \mathrm{~nm}$ are listed).

Table S7: B3LYP TDDFT-predicted energies and expansion coefficients for compound 4 (only excited states with $\mathrm{f}>0.05$ and $\lambda>270 \mathrm{~nm}$ are listed).

Table S8: B3LYP TDDFT-predicted energies and expansion coefficients for compound $\mathbf{1 B F}_{2}$ (only excited states with $\mathrm{f}>0.05$ and $\lambda>270 \mathrm{~nm}$ are listed).

Table S9: B3LYP TDDFT-predicted energies and expansion coefficients for compound $\mathbf{2} \mathbf{B F}_{2}$ (only excited states with $\mathrm{f}>0.05$ and $\lambda>270 \mathrm{~nm}$ are listed).

Table S10: B3LYP TDDFT-predicted energies and expansion coefficients for compound $3 \mathbf{B F}_{2}$ (only excited states with $\mathrm{f}>0.05$ and $\lambda>270 \mathrm{~nm}$ are listed).

Table S11: B3LYP TDDFT-predicted energies and expansion coefficients for compound $\mathbf{4 B F} 2$ (only excited states with $\mathrm{f}>0.05$ and $\lambda>270 \mathrm{~nm}$ are listed).

Table S12: B3LYP DFT excited state optimized geometry of compound 1.

Table S13: B3LYP DFT excited state optimized geometry of compound 2.

Table S14: B3LYP DFT excited state optimized geometry of compound 3.

Table S15: B3LYP DFT excited state optimized geometry of compound 4.

Table S16: B3LYP DFT excited state optimized geometry of compound $\mathbf{1 B F} 2$.

Table S17: B3LYP DFT excited state optimized geometry of compound $\mathbf{2 B F} 2$.

Table S18: B3LYP DFT excited state optimized geometry of compound 3BF2.

Table S19: B3LYP DFT excited state optimized geometry of compound $\mathbf{4} \mathbf{B F}_{\mathbf{2}}$. 


\section{General Information}

\section{Experimental}

All reagents and starting materials were purchased from commercial vendors and used without further purification. 1,3-diiminoisoindoline (DII), and 3-iminoisoindolinone were synthesized according to previously published procedures. ${ }^{1,2}$ Ligand $\mathbf{1}$ was synthesized according to the previously published procedure. ${ }^{3}$ The characterization of $\mathbf{4} \mathbf{B} \mathbf{F}_{\mathbf{2}}$ matches the characterization from the previously published manuscript. ${ }^{4}$ Deuterated solvents were purchased from Cambridge Isotope Laboratories and used as received.

NMR spectra were recorded on $300 \mathrm{MHz}$ and $500 \mathrm{MHz}$ spectrometers and chemical shifts were given in ppm relative to residual solvent resonances $\left({ }^{1} \mathrm{H}\right.$ NMR and ${ }^{13} \mathrm{C}$ NMR spectra). Highresolution mass spectrometry experiments were performed on a Bruker MicroTOF-III and MicroTOF-qIII instruments. Infrared spectra were collected on Thermo Scientific Nicolet iS5 that was equipped with an iD5 ATR. UV-visible spectra were recorded on a Shimadzu UV-2600 UVvisible spectrometer. Fluorescence emission data in solution were recorded on a Horiba JobinYvon FluoroMax-4 fluorescence spectrophotometer using fluorescein in $0.1 \mathrm{M} \mathrm{NaOH}$ as a standard. All slit widths were held constant at $2 \mathrm{~nm}$. The quantum yields in solution were calculated using

the following equation: $\Phi_{X}=\Phi_{S T}\left(\frac{\operatorname{Grad}_{X}}{\operatorname{Grad}_{S T}}\right) *\left(\frac{\eta_{X}^{2}}{\eta_{S T}^{2}}\right) ; \eta_{S T}=1.333, \Phi_{S T}=0.79 ; \eta_{X}=1.431$, and $\operatorname{Grad}$ the gradient from the plot of integrated fluorescence intensity vs absorbance. ${ }^{5}$

Xray intensity data were measured on a Bruker CCD-based and PHOTON II CPAD-based diffractometer with dual $\mathrm{Cu} / \mathrm{Mo} \mathrm{ImuS}$ microfocus optics $(\mathrm{Cu} \mathrm{K} \alpha$ radiation, $\lambda=1.54178 \AA$, Mo $\mathrm{K} \alpha$ radiation, $\lambda=0.71073 \AA$ ). Crystals were mounted on a cryoloop using Paratone oil and placed under a steam of nitrogen at $100 \mathrm{~K}$ (Oxford Cryosystems). The detector was placed at a distance of $5.00 \mathrm{~cm}$ from the crystal. The data were corrected for absorption with the SADABS program. 
The structures were refined using the Bruker SHELXTL Software Package (Version 6.1$){ }^{6}$ and were solved using direct methods until the final anisotropic full-matrix, least squares refinement of $\mathrm{F}^{2}$ converged.

Electrochemistry measurements were conducted using a $\mathrm{CHI} 820 \mathrm{D}$ potentiostat in a standard three-electrode configuration. Platinum wire was used as the counter electrode. The working electrode used was a $2 \mathrm{~mm}$ diameter platinum disk. A nonaqueous $\mathrm{Ag} / \mathrm{Ag}^{+}$reference electrode was used by immersing silver wire in a degassed DMF solution of $0.01 \mathrm{M} \mathrm{AgNO} / 0.1$ $\mathrm{M}$ tetrabutylammonium hexafluorophosphate $\left(\mathrm{TBAPF}_{6}\right)$. All potentials were referenced to the ferrocene/ferrocenium couple. The concentration of analyte was $1.0 \mathrm{mM}$, and the supporting electrolyte was 0.1 M TBAPF6 dissolved in DMF.

\section{Computational Details}

The starting geometries of compounds $\mathbf{1 - 4}$ and $\mathbf{1} \mathbf{B F}_{\mathbf{2}}-\mathbf{4} \mathbf{B} \mathbf{F}_{\mathbf{2}}$ were optimized using a B3LYP exchange-correlation functional. ${ }^{7}$ Energy minima in optimized geometry were confirmed by the frequency calculations (absence of the imaginary frequencies). The solvent effect was modeled using the polarized continuum model $(\mathrm{PCM}){ }^{8}$ In all calculations, DMF was used as the solvent. In PCM-TDDFT calculation, the first 50 states were calculated. All atoms were modeled using the 6-311G(d) ${ }^{9}$ basis set. Gaussian 09 software was used in all calculations. ${ }^{10}$ The QMForge program was used for molecular orbital analysis in all cases. ${ }^{11}$ 


\section{Full citation for Gaussian}

Gaussian 09, Revision D.01,. Frisch, M. J; Trucks, G. W.; Schlegel, H. B.; Scuseria, G. E.; Robb, M. A.; Cheeseman, J. R.; Montgomery, Jr., J. A.; Vreven, T.; Kudin, K. N.; Burant, J. C.; Millam, J. M.; Iyengar, S. S.; Tomasi, J.; Barone, V.; Mennucci, B.; Cossi, M.; Scalmani, G.; Rega, N.; Petersson, G. A.; Nakatsuji, H.; Hada, M.; Ehara, M.; Toyota, K.; Fukuda, R.; Hasegawa, J.; Ishida, M.; Nakajima, T.; Honda, Y.; Kitao, O.; Nakai, H.; Klene, M.; Li, X.; Knox, J. E.;

Hratchian, H. P.; Cross, J. B.; Adamo, C.; Jaramillo, J.; Gomperts, R.; Stratmann, R. E.; Yazyev, O.; Austin, A. J.; Cammi, R.; Pomelli, C.; Ochterski, J. W.; Ayala, P. Y.; Morokuma, K.; Voth, G. A.; Salvador, P.; Dannenberg, J. J.;. Zakrzewski, V. G.; Dapprich, S.; Daniels, A. D.; Strain, M. C.; Farkas, O.; Malick, D. K.; Rabuck, A. D.; Raghavachari, K.; Foresman, J. B.; Ortiz, J. V.; Cui, Q.; Baboul, A. G.; Clifford, S.;. Cioslowski, J.; Stefanov, B. B.; Liu, G.;. Liashenko, A.; Piskorz, P.; Komaromi, I.; Martin, R. L.; Fox, D. J.; Keith, T.; Al-Laham, M. A.; Peng, C. Y.; Nanayakkara, A.; Challacombe, M.; Gill, P. M. W.; Johnson, B.; Chen, W.; Wong, M. W.; Gonzalez, C.; Pople, J. A. Gaussian, Inc., Wallingford CT, 2009. 


\section{Syntheses.}

Synthesis of 2. 1,3-Diiminoisoindoline (1.00 g, $6.89 \mathrm{mmol}), 1 \mathrm{H}$-indazole-3-amine (0.92 g, 6.89 $\mathrm{mmol})$, and $\mathrm{EtOH}(10 \mathrm{~mL})$ were combined in a RBF and heated in an oil bath until all solids were dissolved. The resultant mixture was stirred at room temperature for 12 hours. The precipitate was filtered, washed with cold $\mathrm{EtOH}$, and air dried to give a yellow powder. Crystals suitable for X-ray diffraction were grown from slow evaporation from DMF.
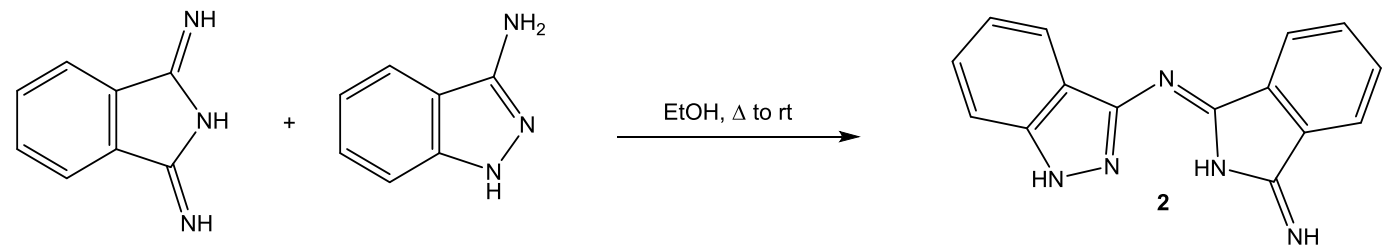

2: Yield: $1.33 \mathrm{~g}(74 \%)$. IR: $1683 \mathrm{~cm}^{-1}\left(v_{\mathrm{CN}}\right) .{ }^{1} \mathrm{H}$ NMR (300 MHz, $\left.\mathrm{d}_{6}-\mathrm{DMSO}\right): \delta=12.87(\mathrm{NH})$, 10.71(NH), $9.09(\mathrm{NH}), 8.85(\mathrm{NH}), 7.96(\mathrm{~s}, 1 \mathrm{H}), 7.83(\mathrm{~m}, 1 \mathrm{H}), 7.67(\mathrm{~s}, 2 \mathrm{H}), 7.56(\mathrm{~m}, 1 \mathrm{H}), 7.45(\mathrm{~s}$, 1H), $7.34(\mathrm{~s}, 1 \mathrm{H}), 7.08(\mathrm{~s}, 1 \mathrm{H}) .{ }^{13} \mathrm{C}\left\{{ }^{1} \mathrm{H}\right\} \mathrm{NMR}\left(125 \mathrm{MHz}, \mathrm{d}_{6}-\mathrm{DMSO}\right): \delta=161.1,150.0,141.2$, 138.9, 131.3, 130.4, 126.1, 121.6, 120.9, 119.6, 117.6, 114.9, 110.2. HRMS (ESI-TOF, positive mode) $\mathrm{m} / \mathrm{z}$ : calcd for $\mathrm{C}_{15} \mathrm{H}_{12} \mathrm{~N}_{5} 262.1087$, found $262.1080[\mathrm{M}+\mathrm{H}]^{+}$.

Synthesis of 3 and 4. The procedure for generating 3 is the same as $\mathbf{4}$ except $1 \mathrm{H}$-indazole-3-amine (0.91 g, $6.84 \mathrm{mmol})$ was used in 4. 3-Iminoisoindolinone (1.00 g, $6.84 \mathrm{mmol})$, 3-aminopyrazole (0.57 g, $6.84 \mathrm{mmol})$, and $\mathrm{EtOH}(10 \mathrm{~mL})$ were combined in a RBF and refluxed in an oil bath for 12 hours. The precipitate was filtered, washed with cold EtOH and air dried to give a white powder for 3 and yellow powder for 4. Crystals suitable for X-ray diffraction were grown from slow evaporation from EtOH. 


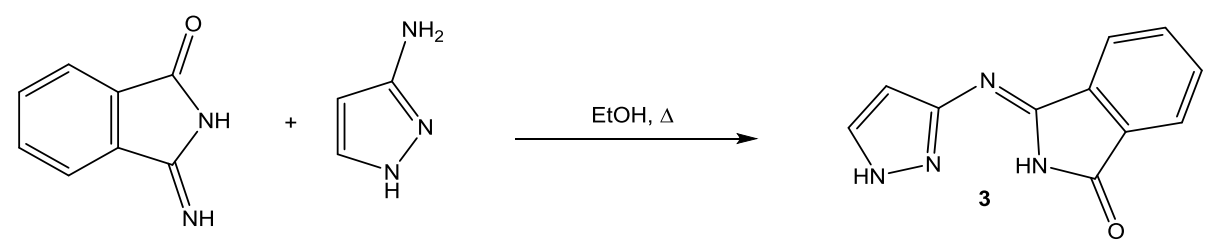

3: Yield: $1.06 \mathrm{~g} \mathrm{(73 \% ).} \mathrm{IR:} 1723 \mathrm{~cm}^{-1}\left(v_{\mathrm{CO}}\right) .{ }^{1} \mathrm{H}$ NMR (300 MHz, $\left.\mathrm{d}_{6}-\mathrm{DMSO}\right): \delta=12.96(\mathrm{~s}, 1 \mathrm{H}$, $\mathrm{NH}), 10.58(\mathrm{~s}, 1 \mathrm{H}, \mathrm{NH}), 7.94(\mathrm{~m}, 1 \mathrm{H}), 7.86-7.70(\mathrm{~m}, 4 \mathrm{H}), 6.40(\mathrm{~m}, 1 \mathrm{H}) .{ }^{13} \mathrm{C}\left\{{ }^{1} \mathrm{H}\right\} \mathrm{NMR}(125 \mathrm{MHz}$, $\left.\mathrm{d}_{6}-\mathrm{DMSO}\right): \delta=168.0,147.6,136.7,133.7,132.0,130.4,139.9,123.1,121.9,102.8$. HRMS (ESITOF, positive mode) $\mathrm{m} / \mathrm{z}$ : calcd for $\mathrm{C}_{11} \mathrm{H}_{9} \mathrm{~N}_{4} \mathrm{O} 213.0771$, found $213.0775[\mathrm{M}+\mathrm{H}]^{+}$.
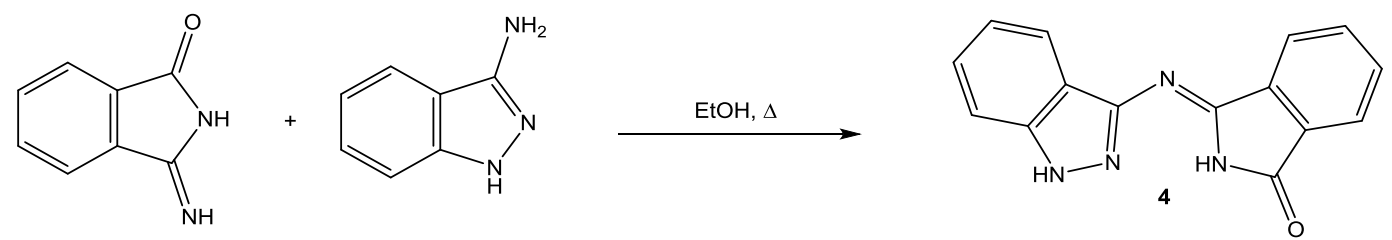

4: Yield: $1.36 \mathrm{~g}(76 \%)$. IR: $1721 \mathrm{~cm}^{-1}\left(v_{\mathrm{CO}}\right) .{ }^{1} \mathrm{H}$ NMR (300 MHz, $\left.\mathrm{d}_{6}-\mathrm{DMSO}\right): \delta=13.15$ (s, $1 \mathrm{H}$, NH), $10.72(\mathrm{~s}, 1 \mathrm{H}, \mathrm{NH}), 8.07(\mathrm{~m}, 1 \mathrm{H}), 7.91-7.74(\mathrm{~m}, 4 \mathrm{H}), 7.53(\mathrm{~m}, 1 \mathrm{H}), 7.43(\mathrm{t}, \mathrm{J}=7.45 \mathrm{~Hz}, 1 \mathrm{H})$, $7.21(\mathrm{t}, \mathrm{J}=7.45 \mathrm{~Hz}, 1 \mathrm{H}) .{ }^{13} \mathrm{C}\left\{{ }^{1} \mathrm{H}\right\} \mathrm{NMR}\left(125 \mathrm{MHz}, \mathrm{d}_{6}-\mathrm{DMSO}\right): \delta=168.1,148.6,148.2,140.7$, 136.5, 133.8, 132.2, 130.4, 127.0, 123.2, 122.0, 120.9, 119.7, 119.4, 110.4. HRMS (ESI-TOF, positive mode) $\mathrm{m} / \mathrm{z}$ : calcd for $\mathrm{C}_{15} \mathrm{H}_{11} \mathrm{~N}_{4} \mathrm{O} 263.0927$, found $263.0940[\mathrm{M}+\mathrm{H}]^{+}$.

Synthesis of $\mathbf{1} \mathbf{B F}_{2}-\mathbf{4} \mathbf{B F}_{2}$. The procedure for generating $\mathbf{1 B F _ { 2 }}$ is the same as $\mathbf{2} \mathbf{B F}_{\mathbf{2}}-\mathbf{4} \mathbf{B F}_{\mathbf{2}}$ except ligand 2 (0.60 g, $2.30 \mathrm{mmol})$ was used in $\mathbf{2 B F}$, ligand $\mathbf{3}(0.49 \mathrm{~g}, 2.30 \mathrm{mmol})$ for $\mathbf{3 B F}$, and ligand 4 (0.60 g, $2.30 \mathrm{mmol})$ for $\mathbf{4 B F}$. Ligand 1 (0.49 g, $2.30 \mathrm{mmol})$ was dissolved in DCM (15 mL) with DIPEA (4 mL, $2.97 \mathrm{~g}, 23.0 \mathrm{mmol}) . \mathrm{BF}_{3}$. $\mathrm{OEt}_{2}(2 \mathrm{~mL}, 2.28 \mathrm{~g}, 16.1 \mathrm{mmol})$ was added dropwise to the solution and the reaction was heated to reflux in an oil bath for 12 hours. The reaction was quenched with water and extracted with DCM. The organic layer was washed with water (3x) and dried over $\mathbf{M g S O}_{4}$. Compounds $\mathbf{1} \mathbf{B F}_{2}$ and $\mathbf{2} \mathbf{B F}_{2}$ were slowly crystallized in DMF (acetone for 
$\mathbf{3} \mathbf{B F}_{2}$ and $\mathbf{4} \mathbf{B F}_{2}$ ), filtered and washed with cold DMF (acetone for $\mathbf{3} \mathbf{B F}_{\mathbf{2}}$ and $\mathbf{4} \mathbf{B F}_{2}$ ). Crystals suitable for X-ray diffraction were grown from these slow evaporation solutions. The characterization of $\mathbf{4} \mathbf{B F}_{2}$ is consistent with the characterization from the previously published manuscript. $^{4}$
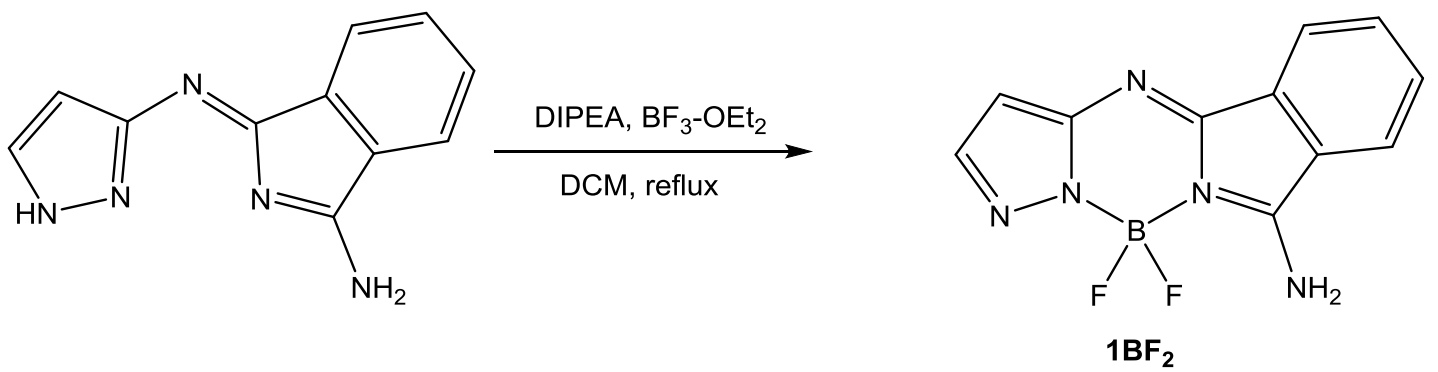

1BF 2 : Yield: $0.39 \mathrm{~g}(65 \%)$. IR: $2925 \mathrm{~cm}^{-1}\left(v_{\mathrm{NH}}\right) 1662 \mathrm{~cm}^{-1}\left(v_{\mathrm{CN}}\right) .{ }^{1} \mathrm{H}$ NMR $\left(300 \mathrm{MHz}, \mathrm{d}_{6}-\right.$ DMSO): $\delta=8.30(\mathrm{~m}, 1 \mathrm{H}), 8.00(\mathrm{~m}, 1 \mathrm{H}), 7.87(\mathrm{~m}, 1 \mathrm{H}), 7.79(\mathrm{~m}, 1 \mathrm{H}), 7.54(\mathrm{~s}, 1 \mathrm{H}), 6.35(\mathrm{~s}$, 1H). ${ }^{13} \mathrm{C}\left\{{ }^{1} \mathrm{H}\right\}$ NMR (125 MHz, $\left.\mathrm{d}_{6}-\mathrm{DMSO}\right): \delta=163.3,149.9,147.3,141.2,134.9,134.6,132.1$, 129.5, 124.4, 122.6, 102.6. ${ }^{19} \mathrm{~F}\left(\mathrm{~d}_{6}-\mathrm{DMSO}, 282 \mathrm{MHz}\right): \delta=-145.15$. HRMS (ESI-TOF, positive mode) $\mathrm{m} / \mathrm{z}$ : calcd for $\mathrm{C}_{11} \mathrm{H}_{9} \mathrm{BF}_{2} \mathrm{~N}_{5} 260.0914$, found $260.0945[\mathrm{M}+\mathrm{H}]^{+}$.
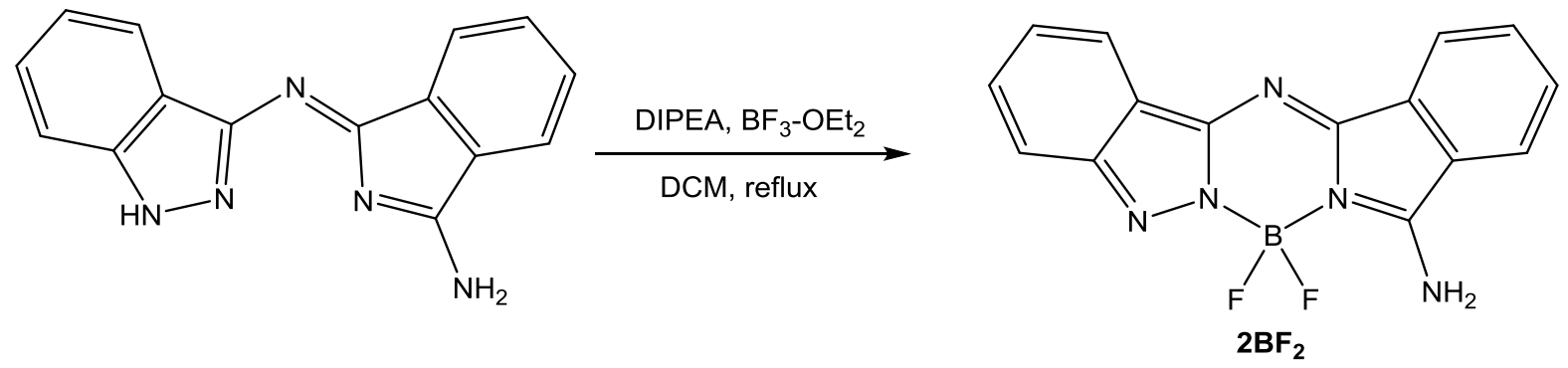

2BF $_{2}:$ Yield: $0.48 \mathrm{~g}(68 \%)$. IR: 3152, $2876 \mathrm{~cm}^{-1}\left(v_{\mathrm{NH}}\right), 1629 \mathrm{~cm}^{-1}\left(v_{\mathrm{CN}}\right) .{ }^{1} \mathrm{H}$ NMR $\left(300 \mathrm{MHz}, \mathrm{d}_{6}\right.$ - DMSO): $\delta=8.33(\mathrm{~d}, \mathrm{~J}=7.34 \mathrm{~Hz}, 1 \mathrm{H}), 8.10(\mathrm{~d}, \mathrm{~J}=7.34 \mathrm{~Hz}, 1 \mathrm{H}), 7.96-7.78(\mathrm{~m}, 3 \mathrm{H}), 7.64(\mathrm{~d}, \mathrm{~J}=$ $8.80 \mathrm{~Hz}, 1 \mathrm{H}), 7.30(\mathrm{t}, \mathrm{J}=7.34 \mathrm{~Hz}, 1 \mathrm{H}), 7.13(\mathrm{t}, \mathrm{J}=7.34 \mathrm{~Hz}, 1 \mathrm{H}) .{ }^{13} \mathrm{C}\left\{{ }^{1} \mathrm{H}\right\} \mathrm{NMR}\left(125 \mathrm{MHz}, \mathrm{d}_{6}-\right.$ DMSO): $\delta=163.1,134.9,134.5,131.8,129.2,126.0,124.5,122.6,122.1,119.6,118.2,117.5 .{ }^{19} \mathrm{~F}$ $\left(\mathrm{d}_{6}-\mathrm{DMSO}, 282 \mathrm{MHz}\right): \delta=-140.41$. HRMS (ESI-TOF, positive mode) $\mathrm{m} / \mathrm{z}$ : calcd for $\mathrm{C}_{11} \mathrm{H}_{9} \mathrm{BF}_{2} \mathrm{~N}_{5}$ 308.0925, found $308.0919[\mathrm{M}-\mathrm{H}]^{-}$. 

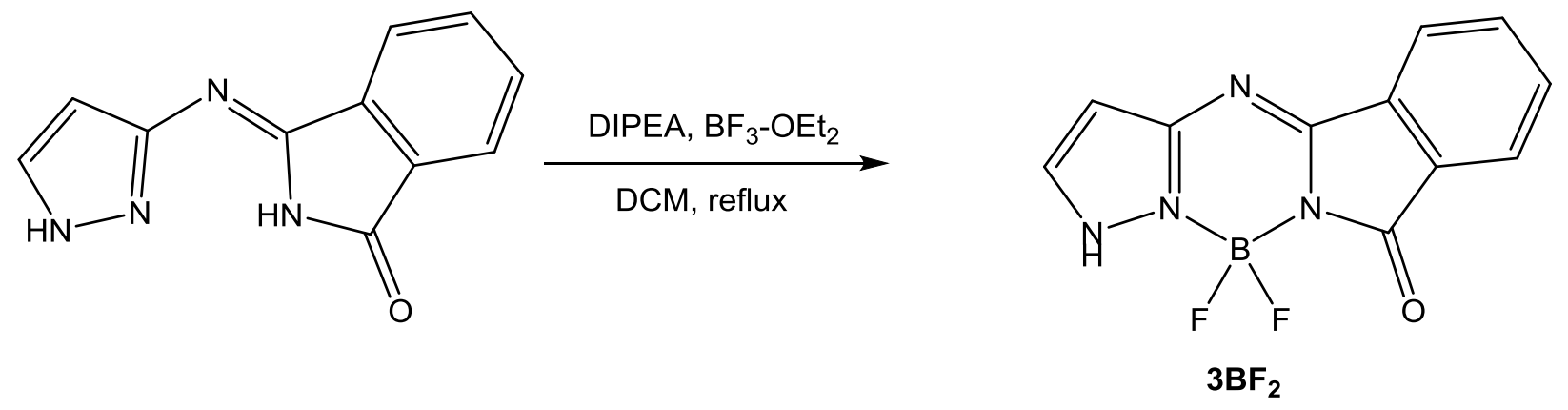

3BF $\boldsymbol{F}_{2}$ : Yield: $0.20 \mathrm{~g}(33 \%)$. IR: $3143 \mathrm{~cm}^{-1}\left(v_{\mathrm{NH}}\right), 1717 \mathrm{~cm}^{-1}\left(v_{\mathrm{CO}}\right) .{ }^{1} \mathrm{H}$ NMR $\left(300 \mathrm{MHz}, \mathrm{d}_{6}-\right.$ DMSO): $\delta=8.24(\mathrm{~s}, 1 \mathrm{H}), 7.96(\mathrm{~d}, 1 \mathrm{H}, \mathrm{J}=6.36 \mathrm{~Hz}), 7.88-7.77(\mathrm{~m}, 3 \mathrm{H}), 6.65(\mathrm{~s}, 1 \mathrm{H}),{ }^{13} \mathrm{C}\left\{{ }^{1} \mathrm{H}\right\}$ NMR (125 MHz, $\left.\mathrm{d}_{6}-\mathrm{DMSO}\right): \delta=170.5,151.6,136.0,133.4,132.3,131.9,123.1,121.58$. HRMS (ESI-TOF, negative mode) m/z: calcd for $\mathrm{C}_{11} \mathrm{H}_{6} \mathrm{BF}_{2} \mathrm{~N}_{4} \mathrm{O} 259.0608$, found $259.0585[\mathrm{M}-\mathrm{H}]^{-}$.
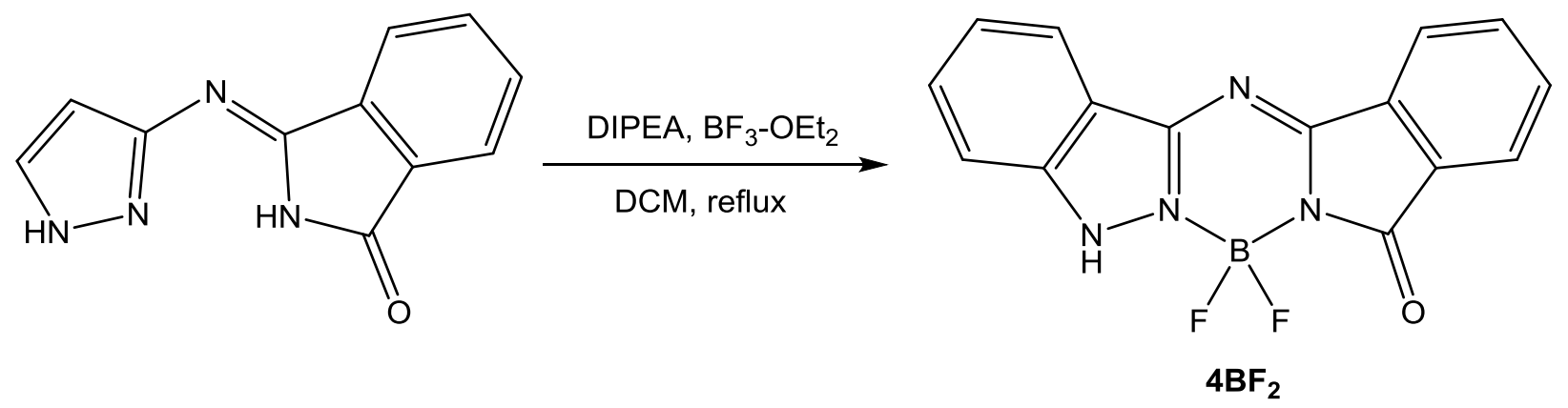

$\boldsymbol{A B F}_{2}$ : Yield: $0.29 \mathrm{~g}(41 \%) .{ }^{1} \mathrm{H}$ NMR (300 MHz, d6 - DMSO): $\delta=8.11(\mathrm{~m}, 2 \mathrm{H}), 7.93-7.73(\mathrm{~m}$, 4H), $7.64(\mathrm{~d}, \mathrm{~J}=8.31 \mathrm{~Hz}, 1 \mathrm{H}), 7.43(\mathrm{t}, \mathrm{J}=7.83 \mathrm{~Hz}, 1 \mathrm{H})$. (ESI-TOF, negative mode) $\mathrm{m} / \mathrm{z}$ : calcd for $\mathrm{C}_{15} \mathrm{H}_{8} \mathrm{BF}_{2} \mathrm{~N}_{4} \mathrm{O}$ 309.0761, found $309.0765[\mathrm{M}-\mathrm{H}]^{-}$. 


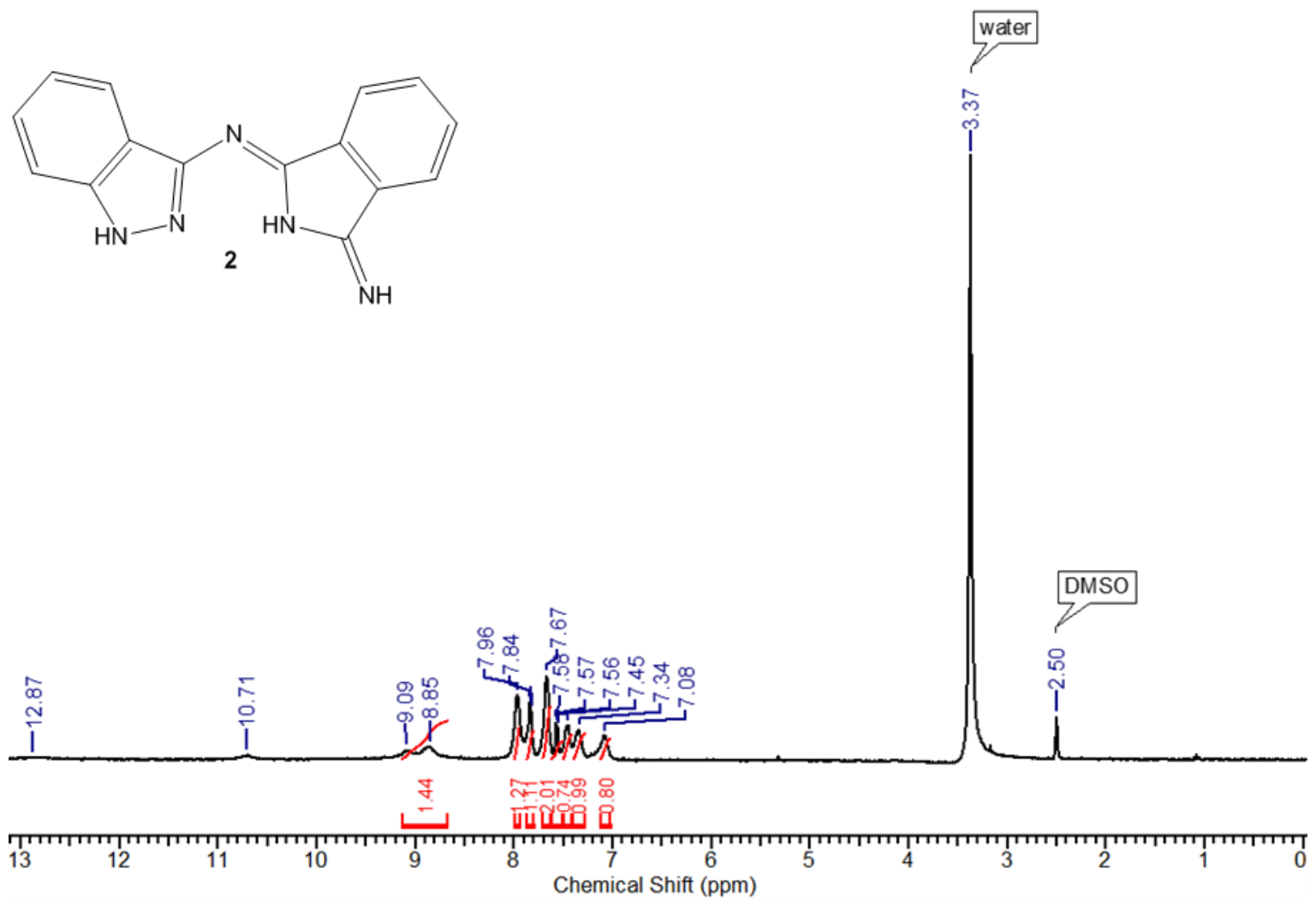

Figure S1: ${ }^{1} \mathrm{H}$ NMR (300 MHz) of 2 in d6-DMSO. 


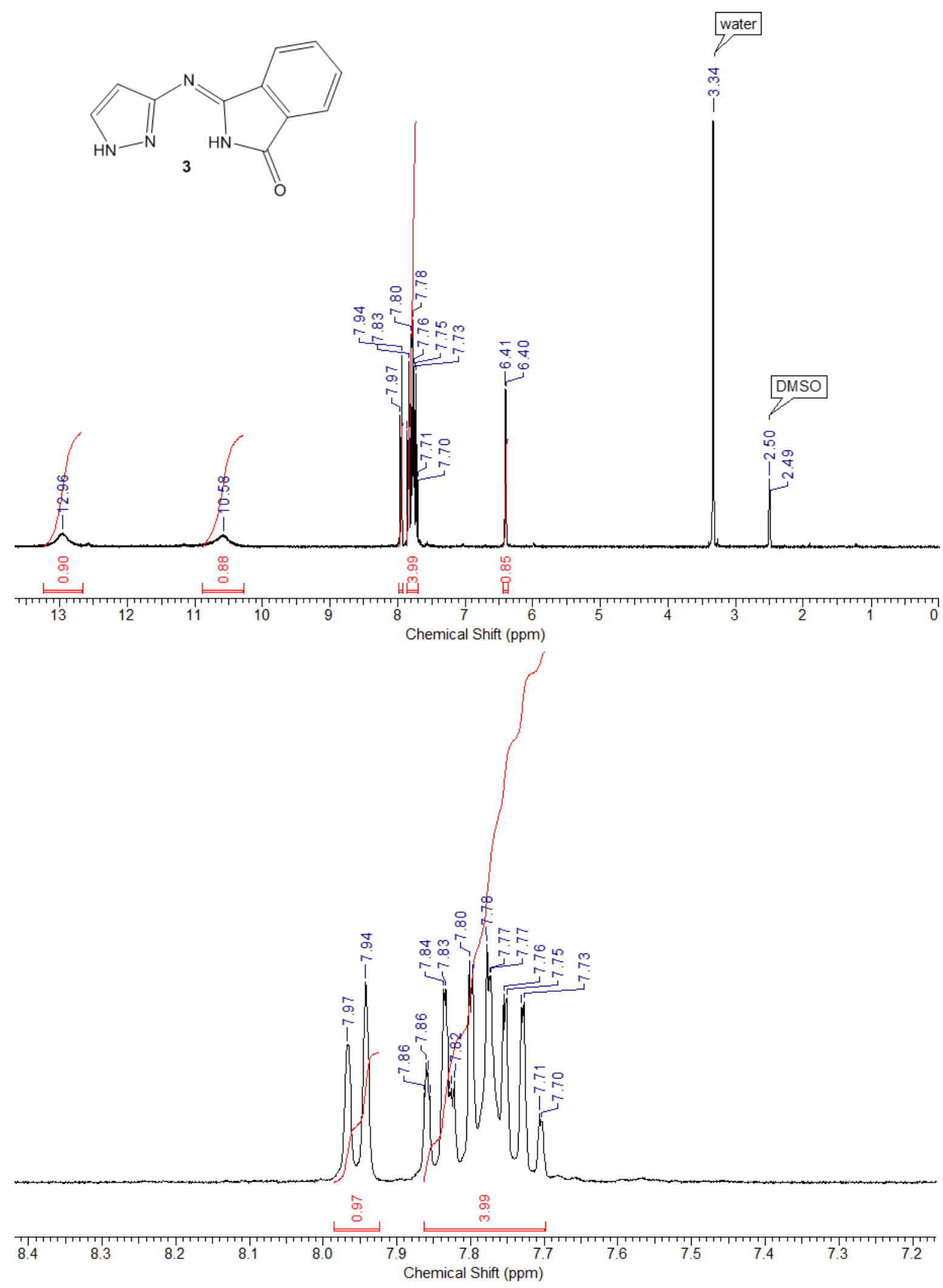

Figure S2: ${ }^{1} \mathrm{H}$ NMR $(300 \mathrm{MHz})$ of $\mathbf{3}$ in d6-DMSO. 

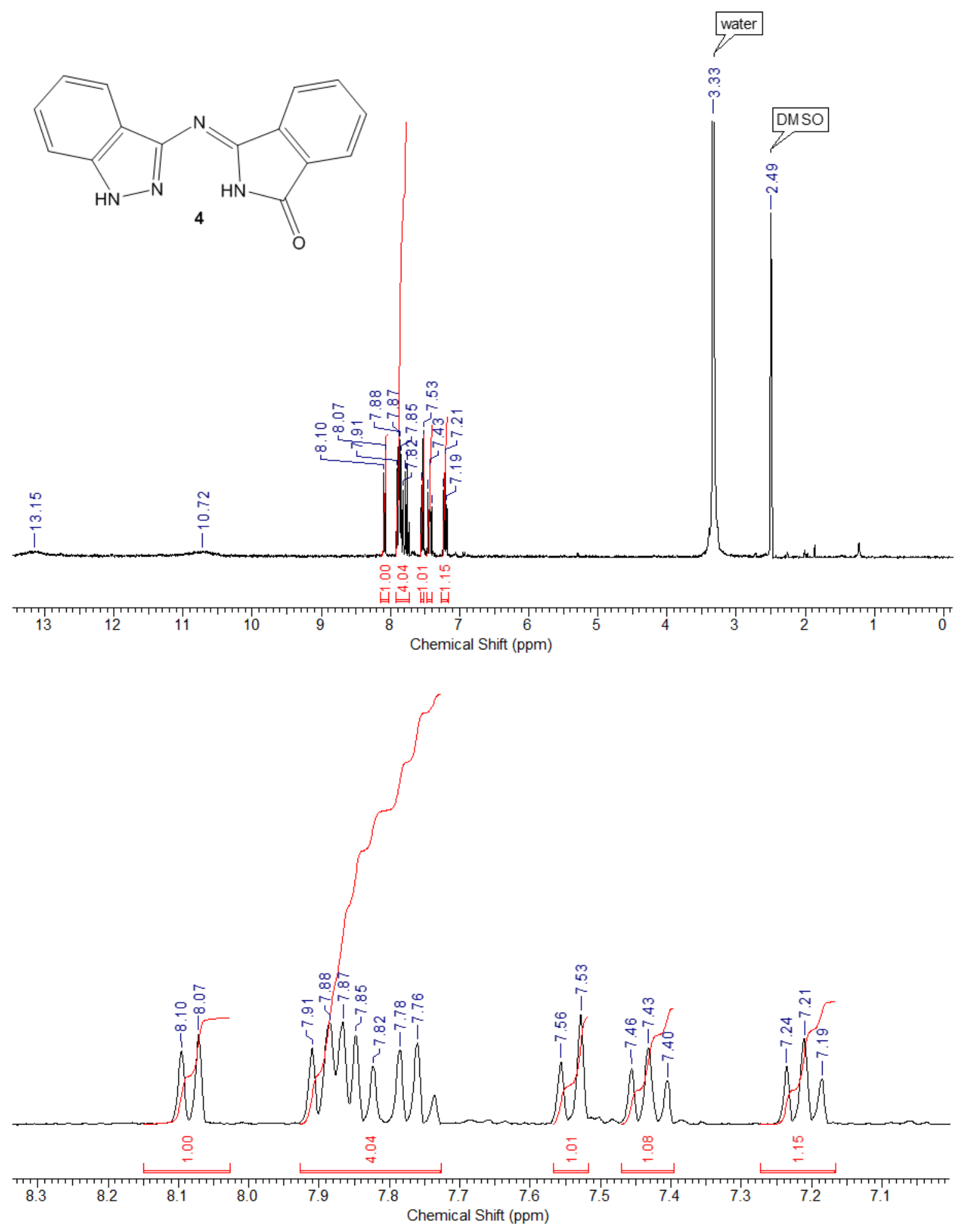

Figure S3: ${ }^{1} \mathrm{H}$ NMR $(300 \mathrm{MHz})$ of 4 in d6-DMSO. 

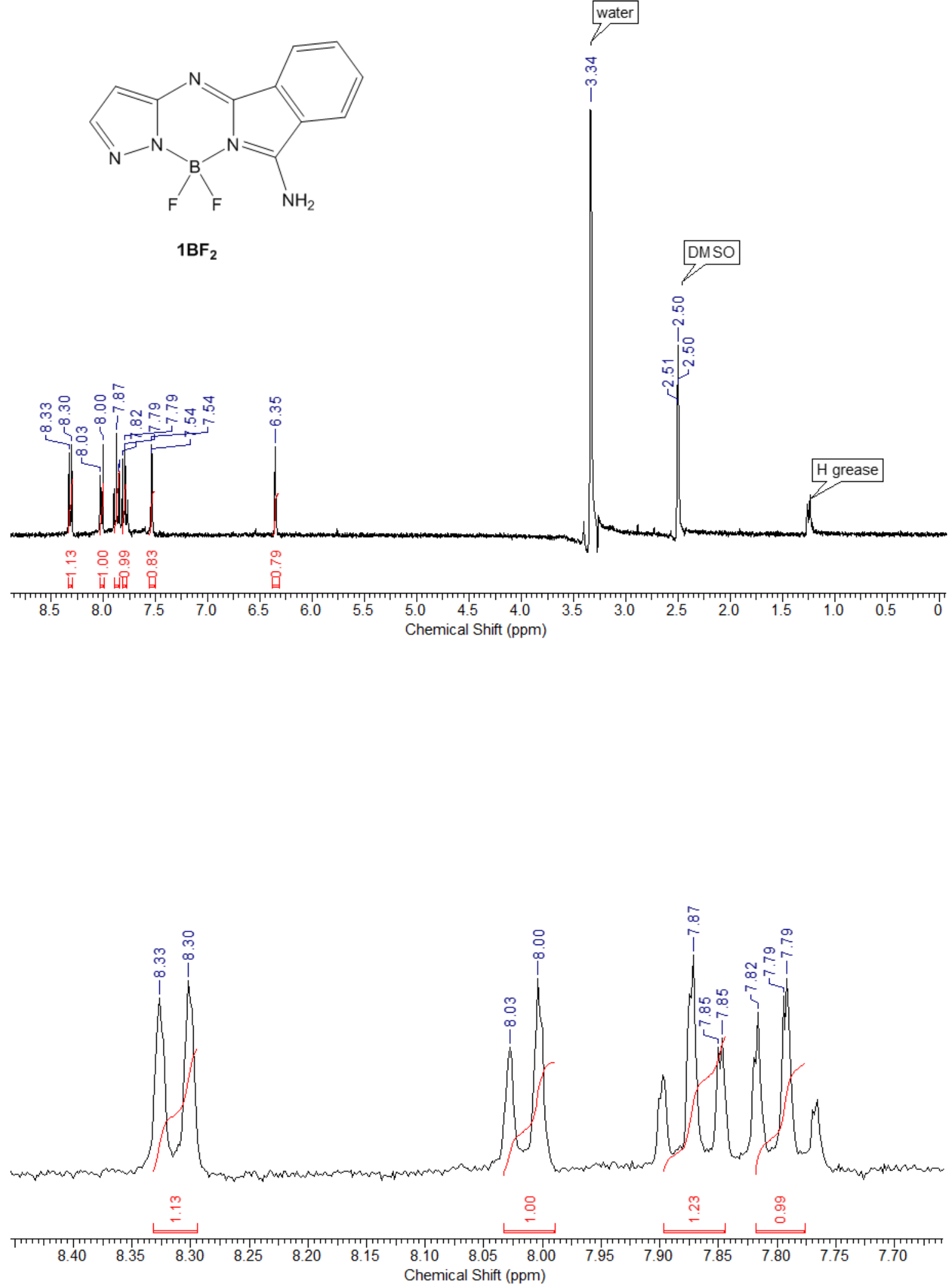

Figure S4: ${ }^{1} \mathrm{H}$ NMR (300 MHz) of $\mathbf{1 B F}_{\mathbf{2}}$ in d6-DMSO. 

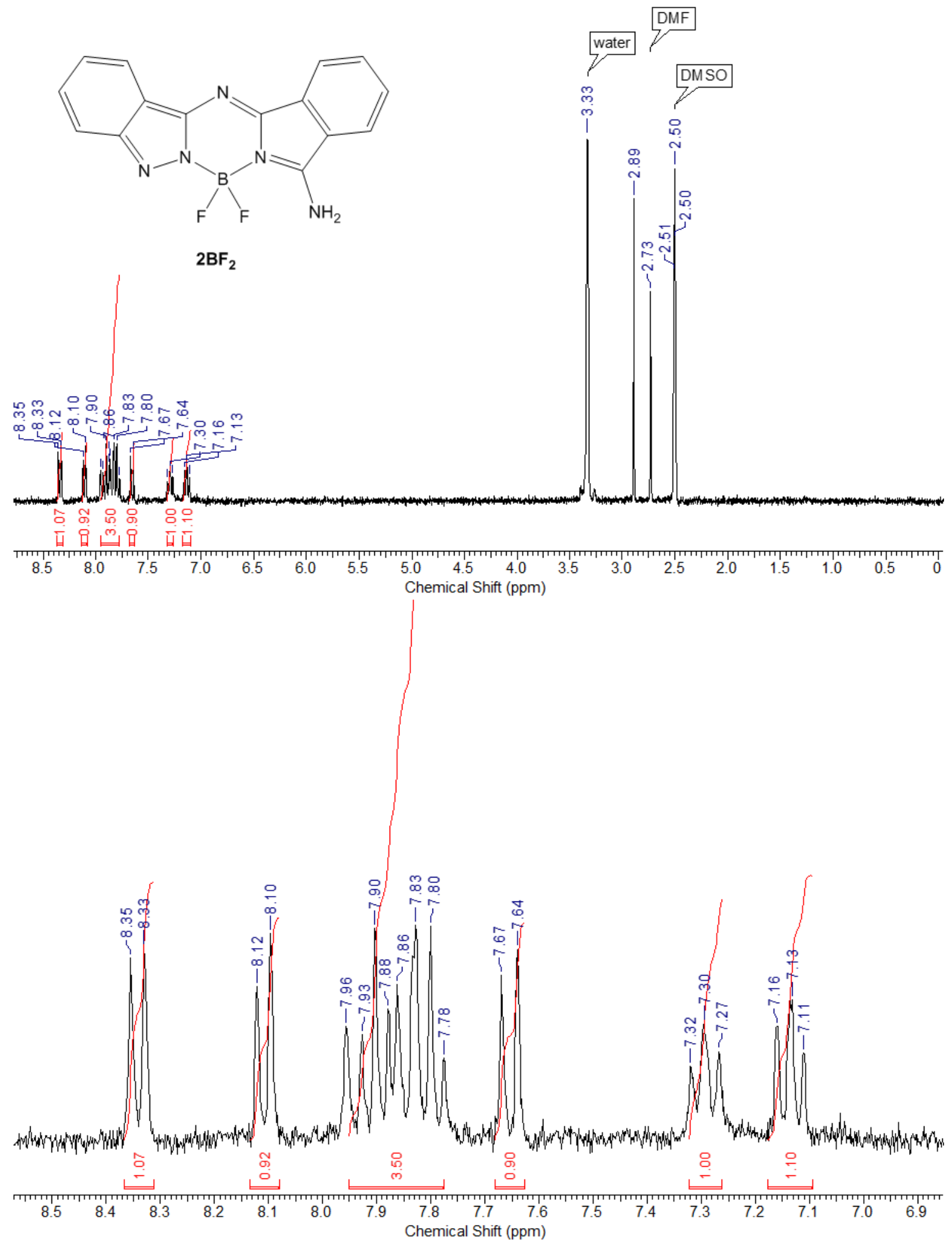

Figure S5: ${ }^{1} \mathrm{H}$ NMR (300 MHz) of $\mathbf{2 B F}_{2}$ in d6-DMSO. 

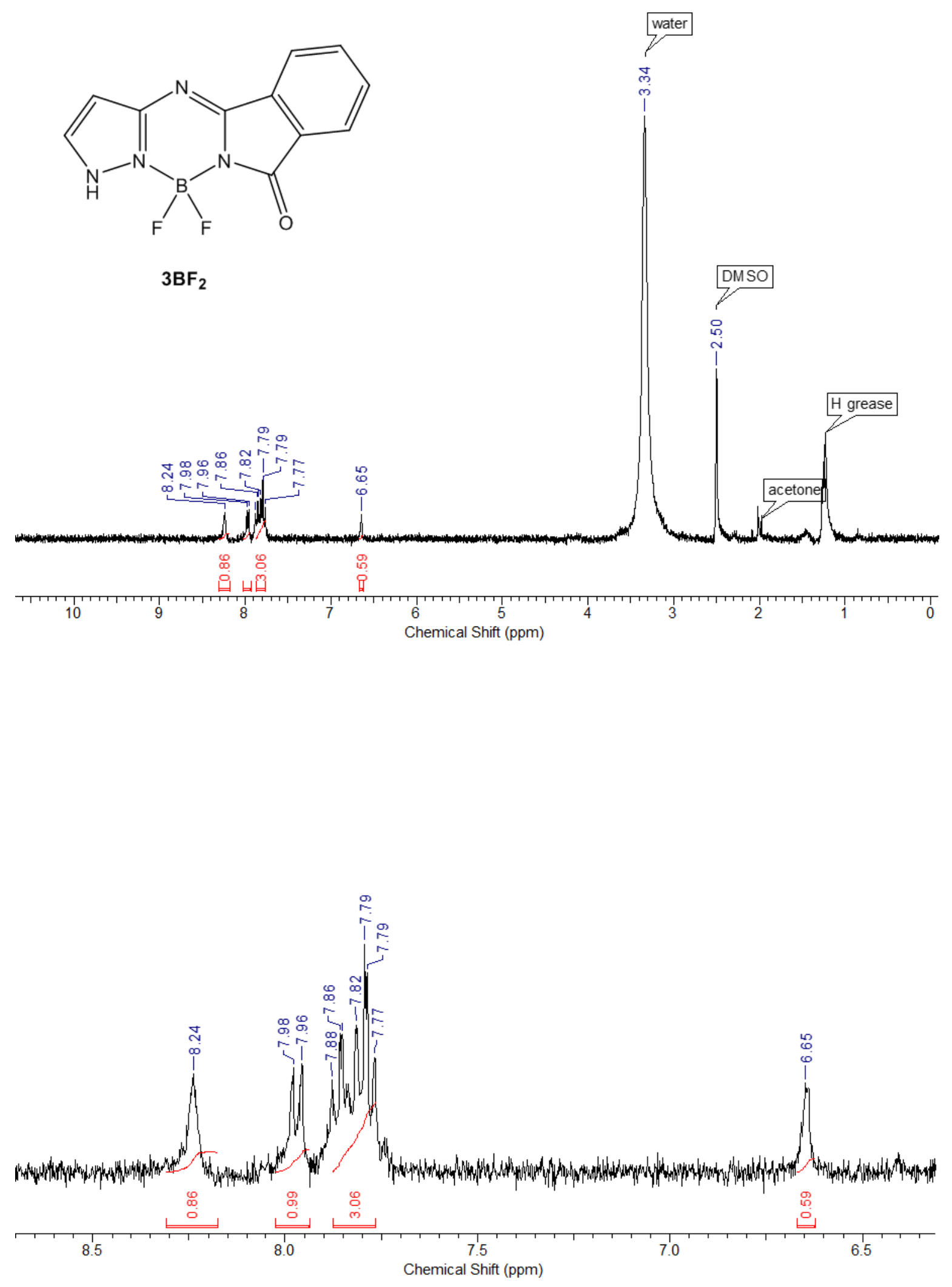

Figure S6: ${ }^{1} \mathrm{H}$ NMR $(300 \mathrm{MHz})$ of $\mathbf{3 B F} \mathbf{2}$ in d6-DMSO. 

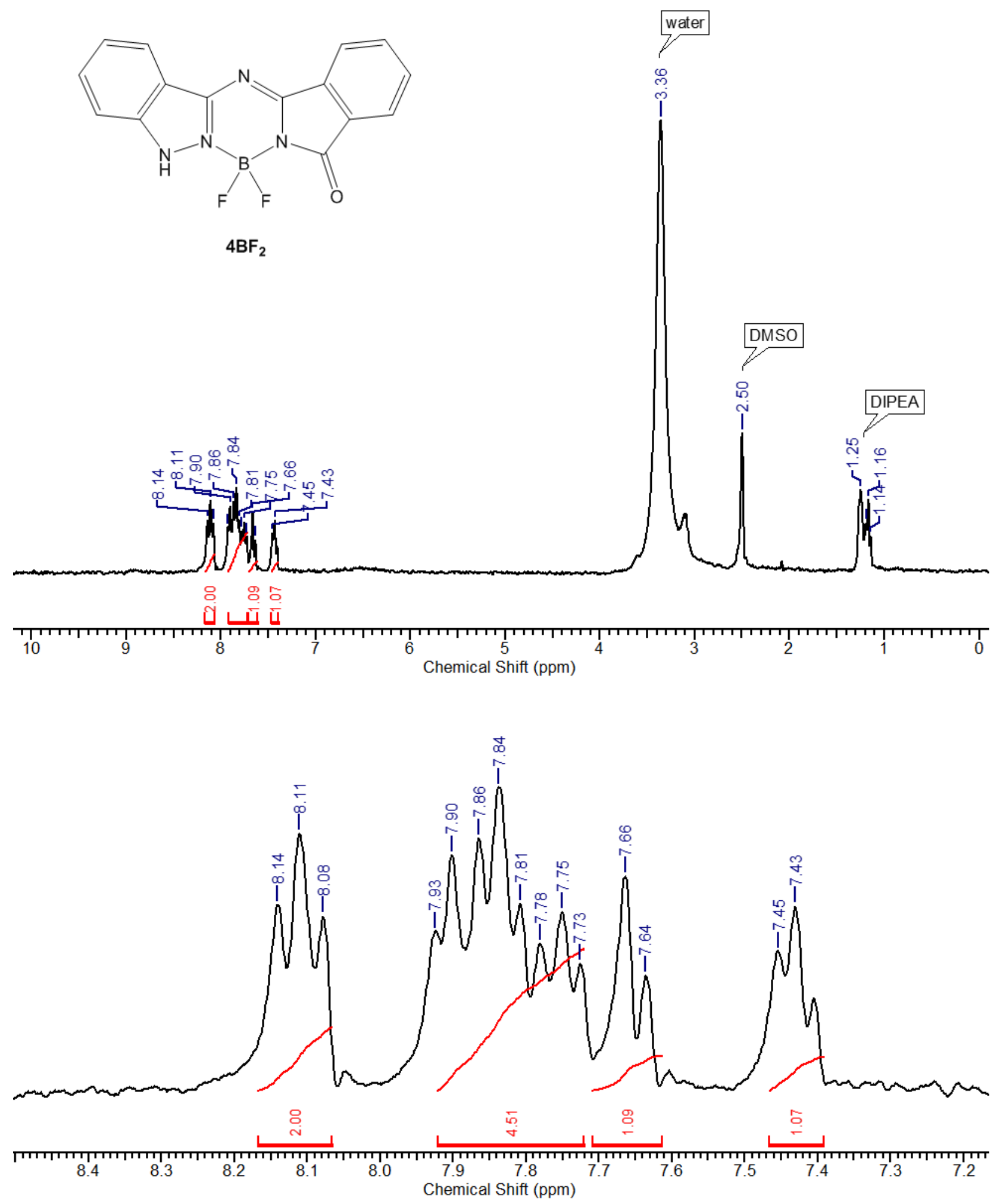

Figure S7: ${ }^{1} \mathrm{H}$ NMR (300 MHz) of $\mathbf{4 B F} \mathbf{2}$ in d6-DMSO. 

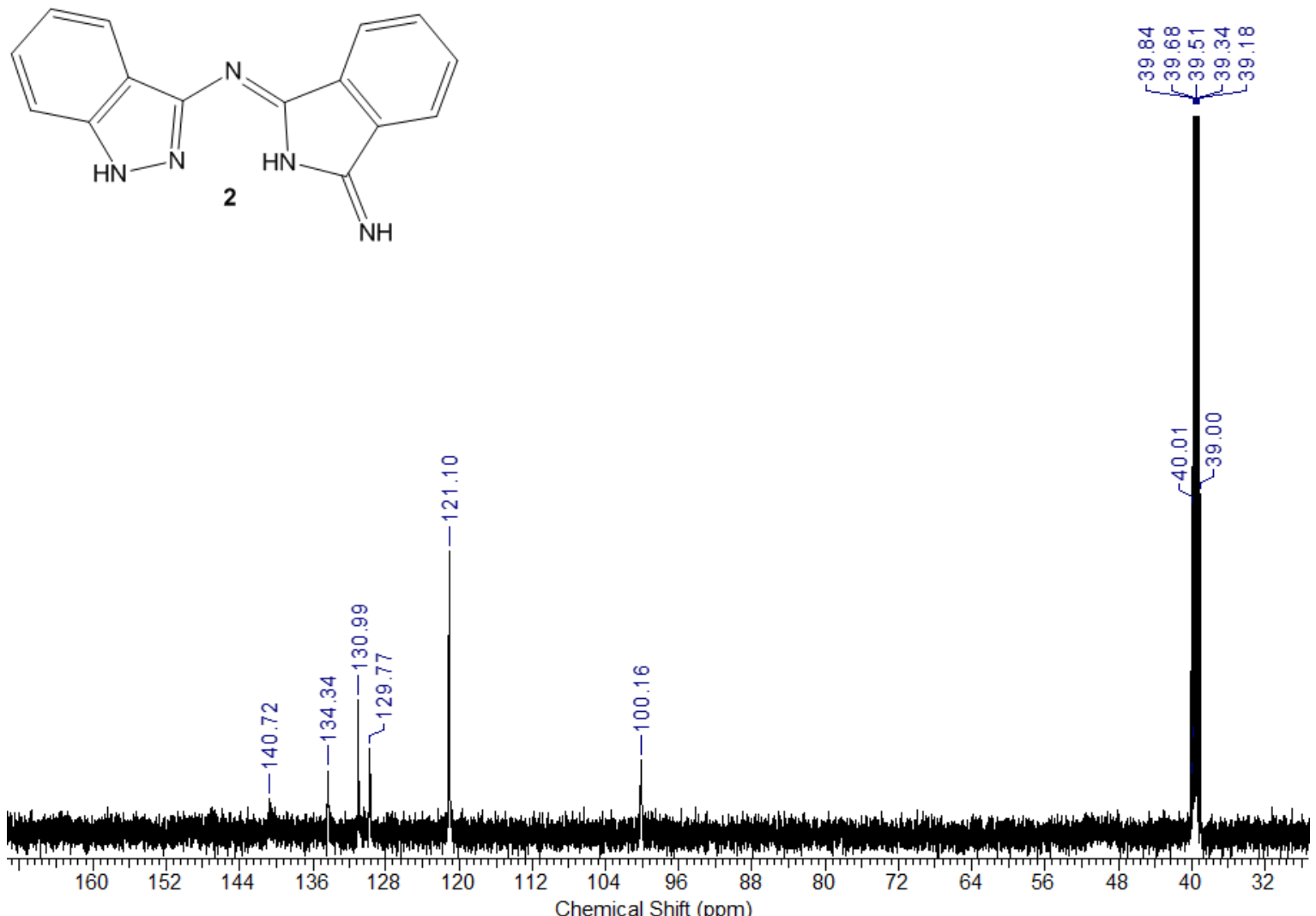

Figure S8: ${ }^{13} \mathrm{C}\left\{{ }^{1} \mathrm{H}\right\}$ NMR $(125 \mathrm{MHz})$ of 2 in d6-DMSO. 

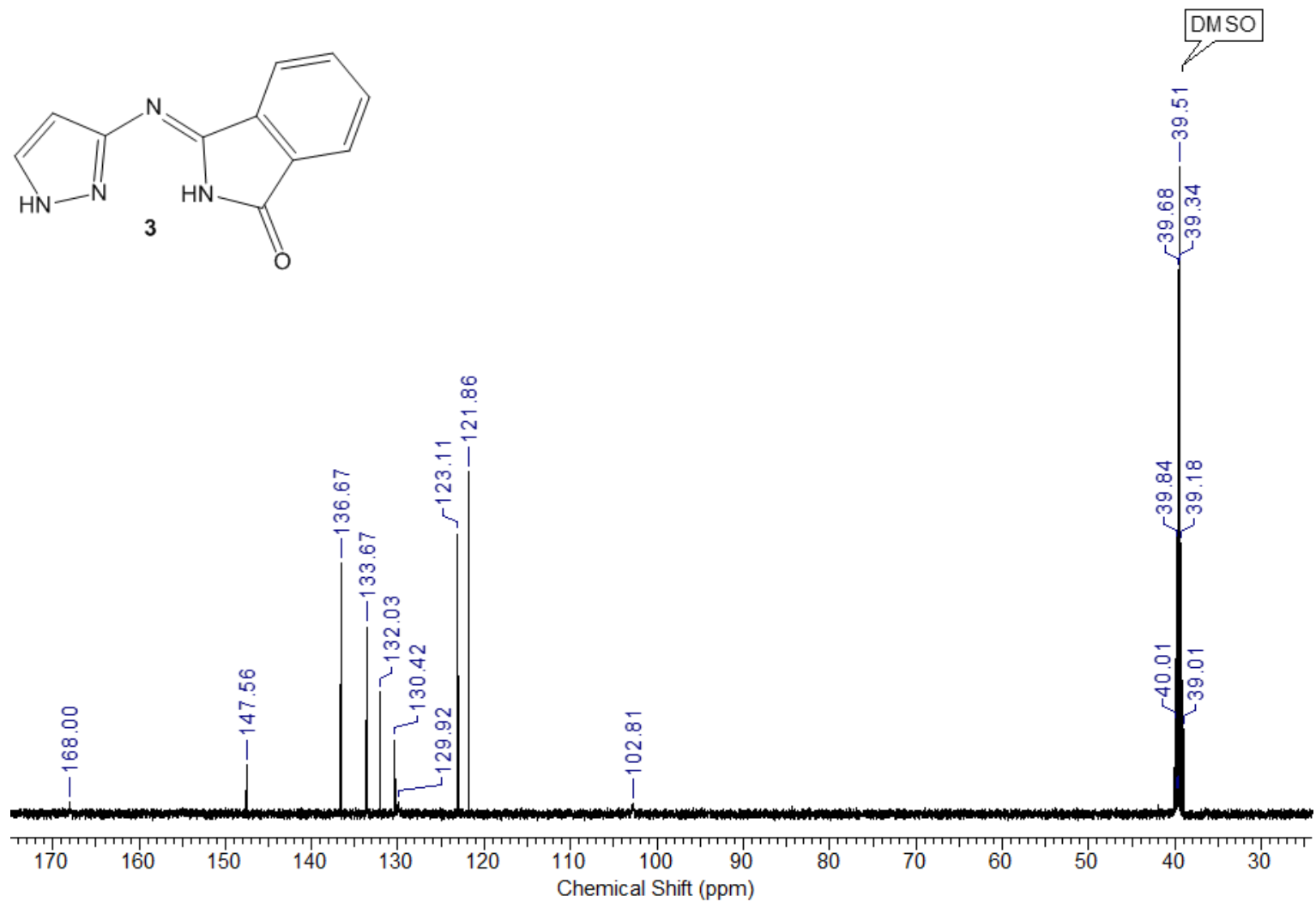

Figure S9: ${ }^{13} \mathrm{C}\left\{{ }^{1} \mathrm{H}\right\}$ NMR $(125 \mathrm{MHz})$ of 3 in d6-DMSO. 

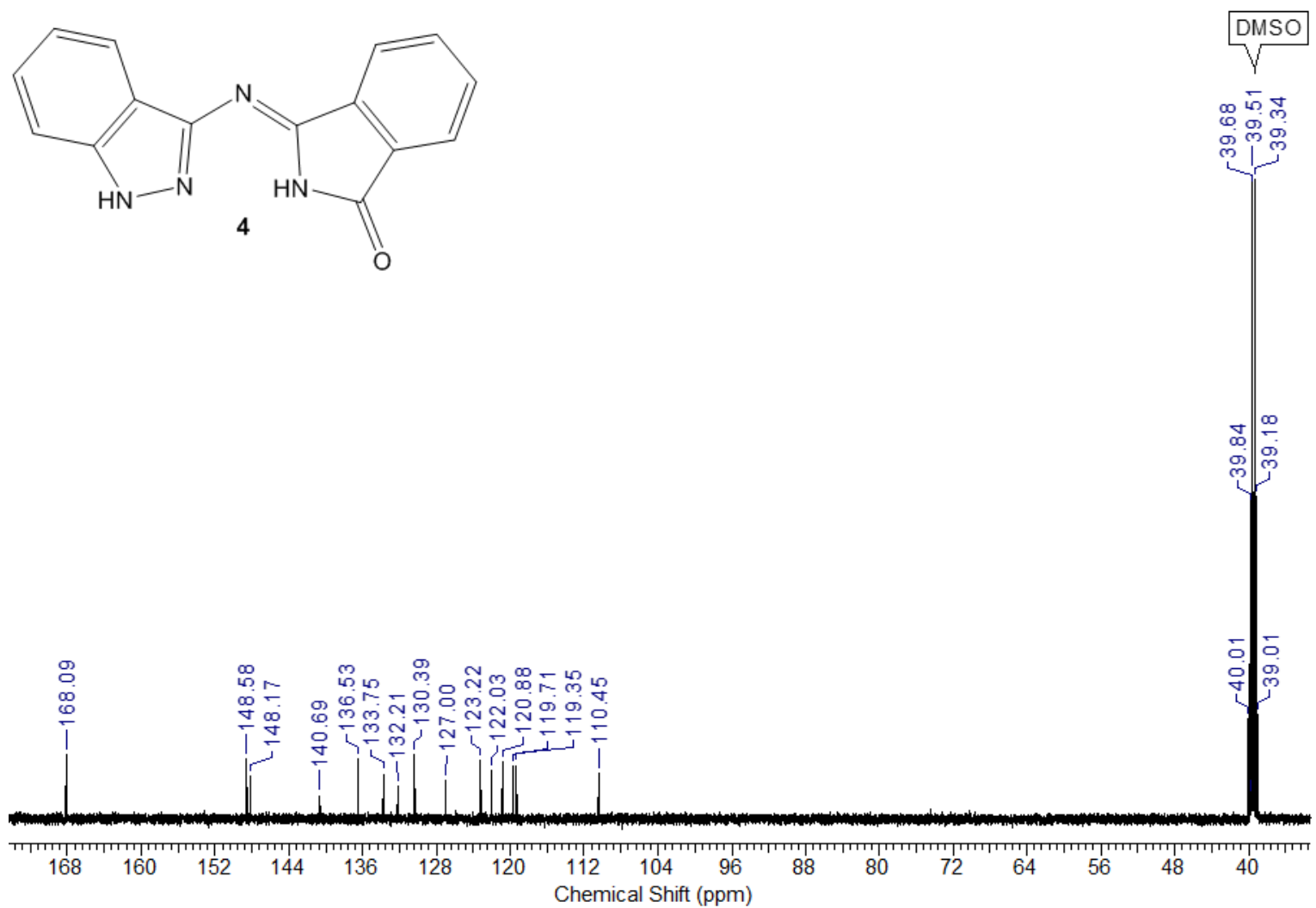

Figure S10: ${ }^{13} \mathrm{C}\left\{{ }^{1} \mathrm{H}\right\}$ NMR (125 MHz) of 4 in d6-DMSO. 


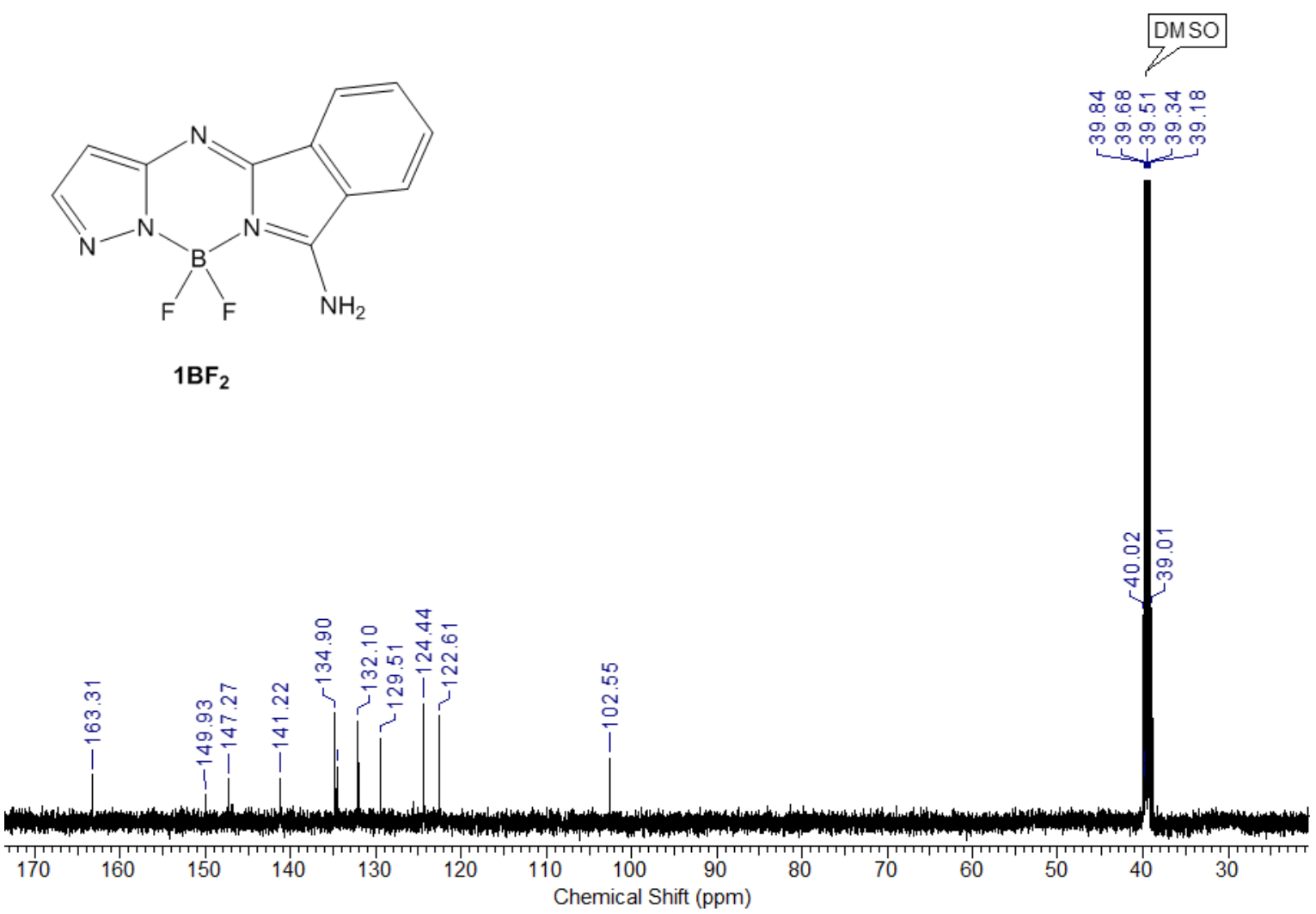

Figure S11: ${ }^{13} \mathrm{C}\left\{{ }^{1} \mathrm{H}\right\}$ NMR $(125 \mathrm{MHz})$ of $\mathbf{1 B F}_{2}$ in d6-DMSO. 


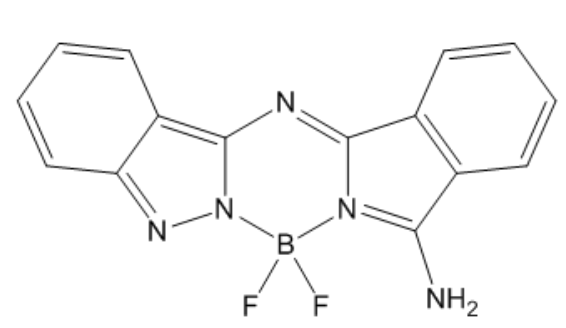

DMSO

$2 \mathrm{BF}_{2}$

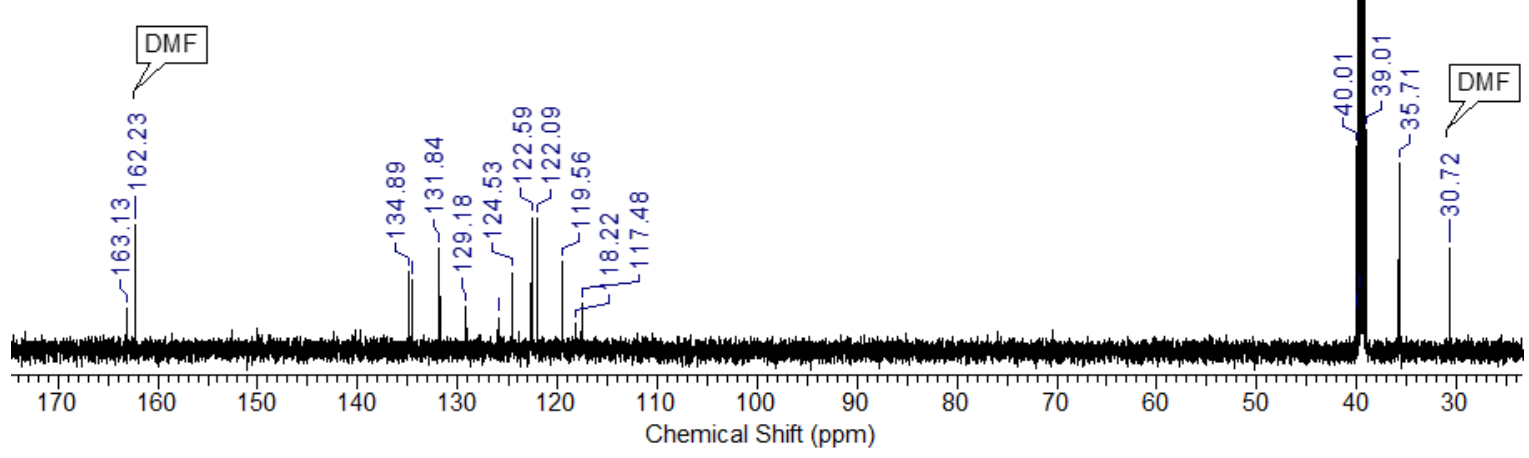

Figure S12: ${ }^{13} \mathrm{C}\left\{{ }^{1} \mathrm{H}\right\}$ NMR (125 MHz) of $\mathbf{2 B F _ { 2 }}$ in d6-DMSO 


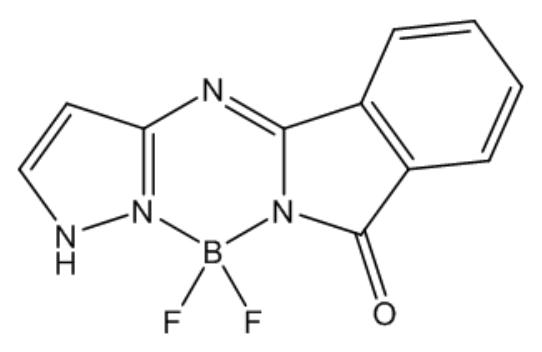

$3 \mathrm{BF}_{2}$

का

ल)

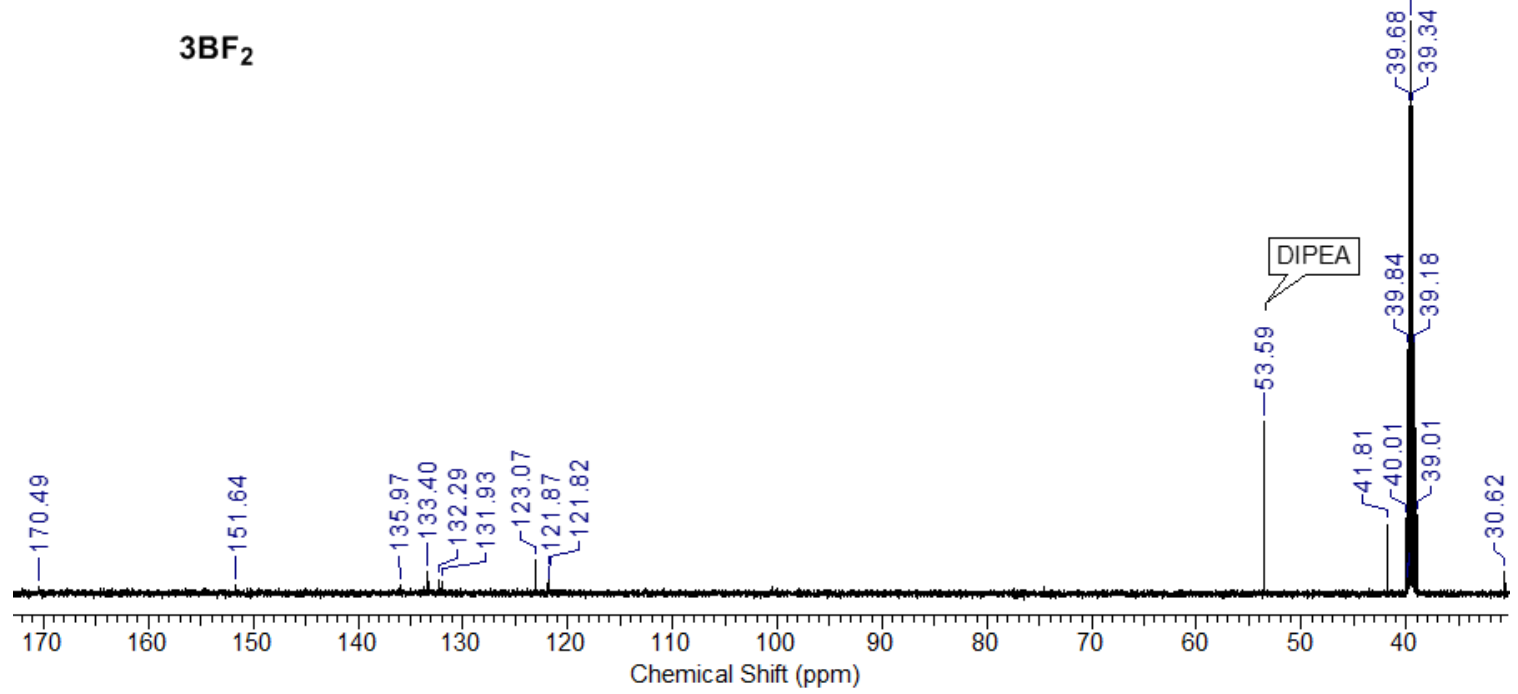

Figure S13: ${ }^{13} \mathrm{C}\left\{{ }^{1} \mathrm{H}\right\}$ NMR $(125 \mathrm{MHz})$ of $\mathbf{3} \mathbf{B F}_{2}$ in d6-DMSO 


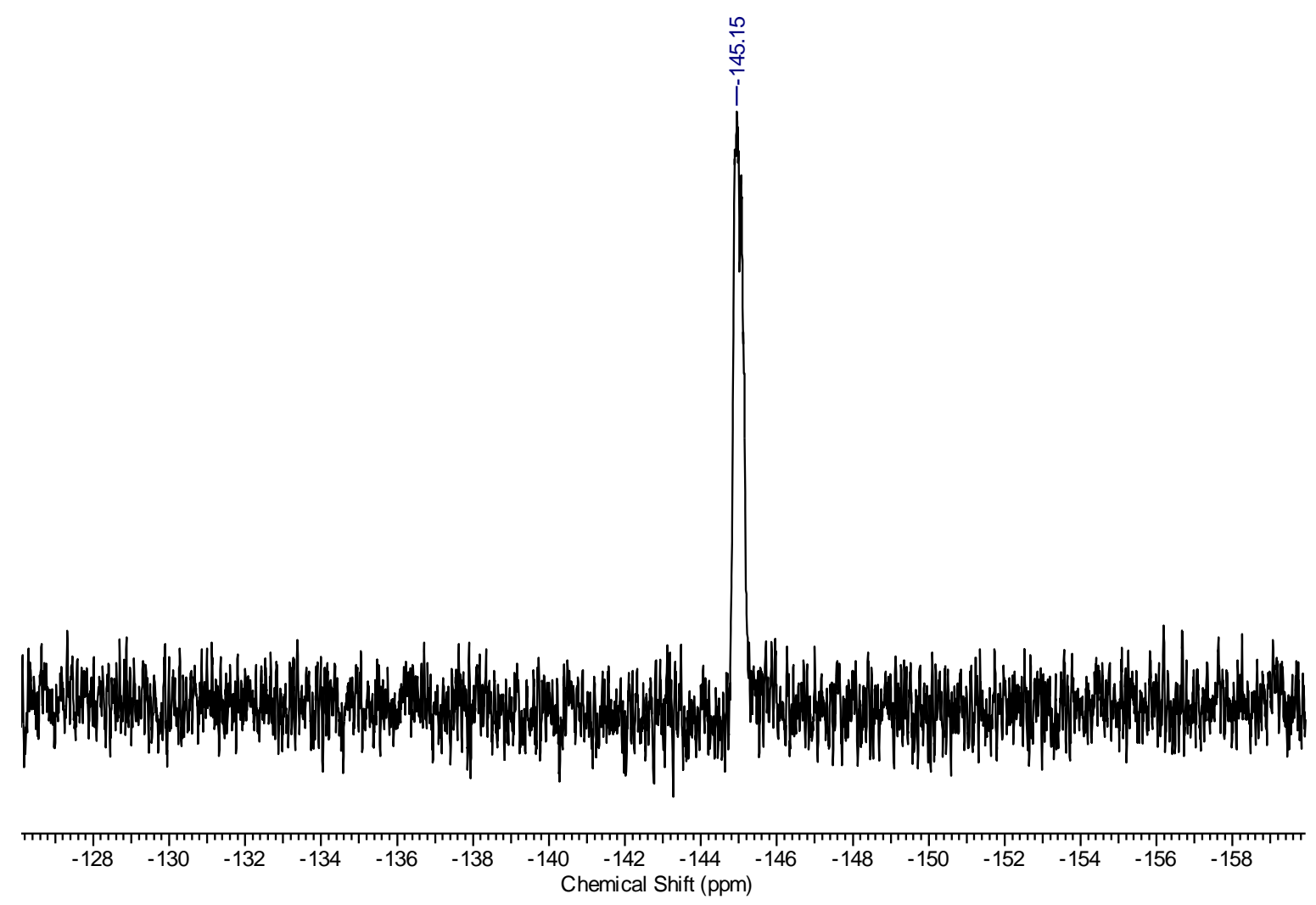

Figure S14: ${ }^{19} \mathrm{~F}$ NMR (282 MHz) of $\mathbf{1 B F} 2$ in d6-DMSO. 


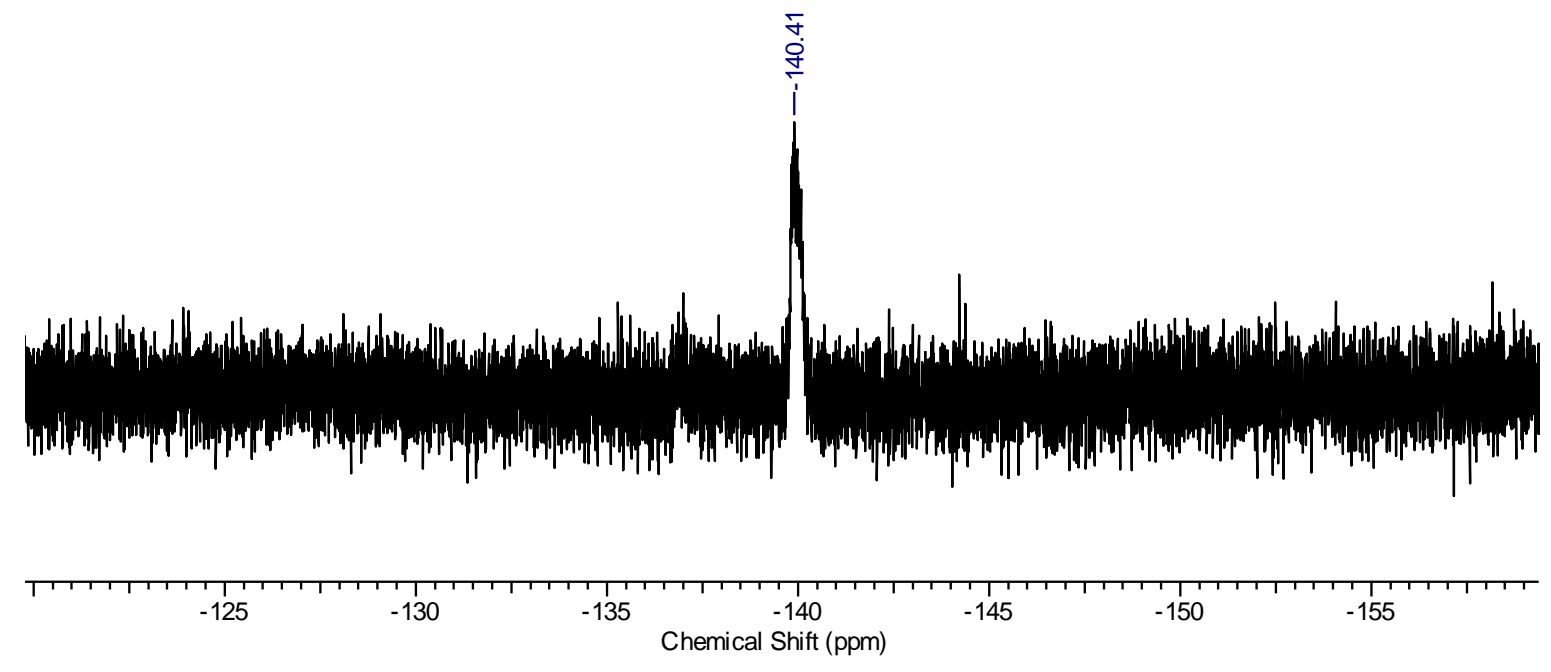

Figure S15: ${ }^{19}$ F NMR (282 MHz) of $\mathbf{2} \mathbf{B F}_{2}$ in d6-DMSO. 


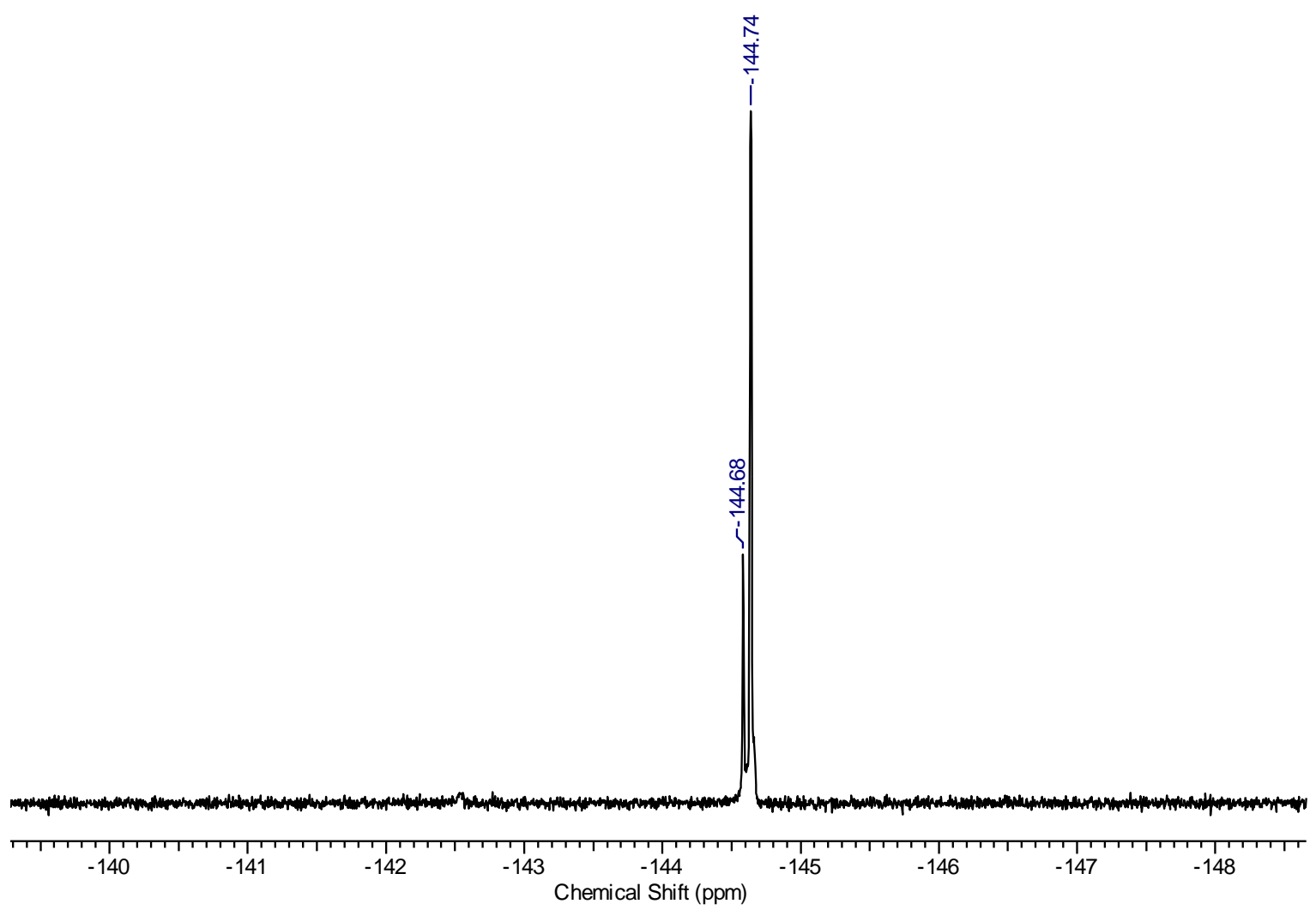

Figure S16: ${ }^{19}$ F NMR (282 MHz) of $\mathbf{3 B F} 2$ in d6-DMSO. 

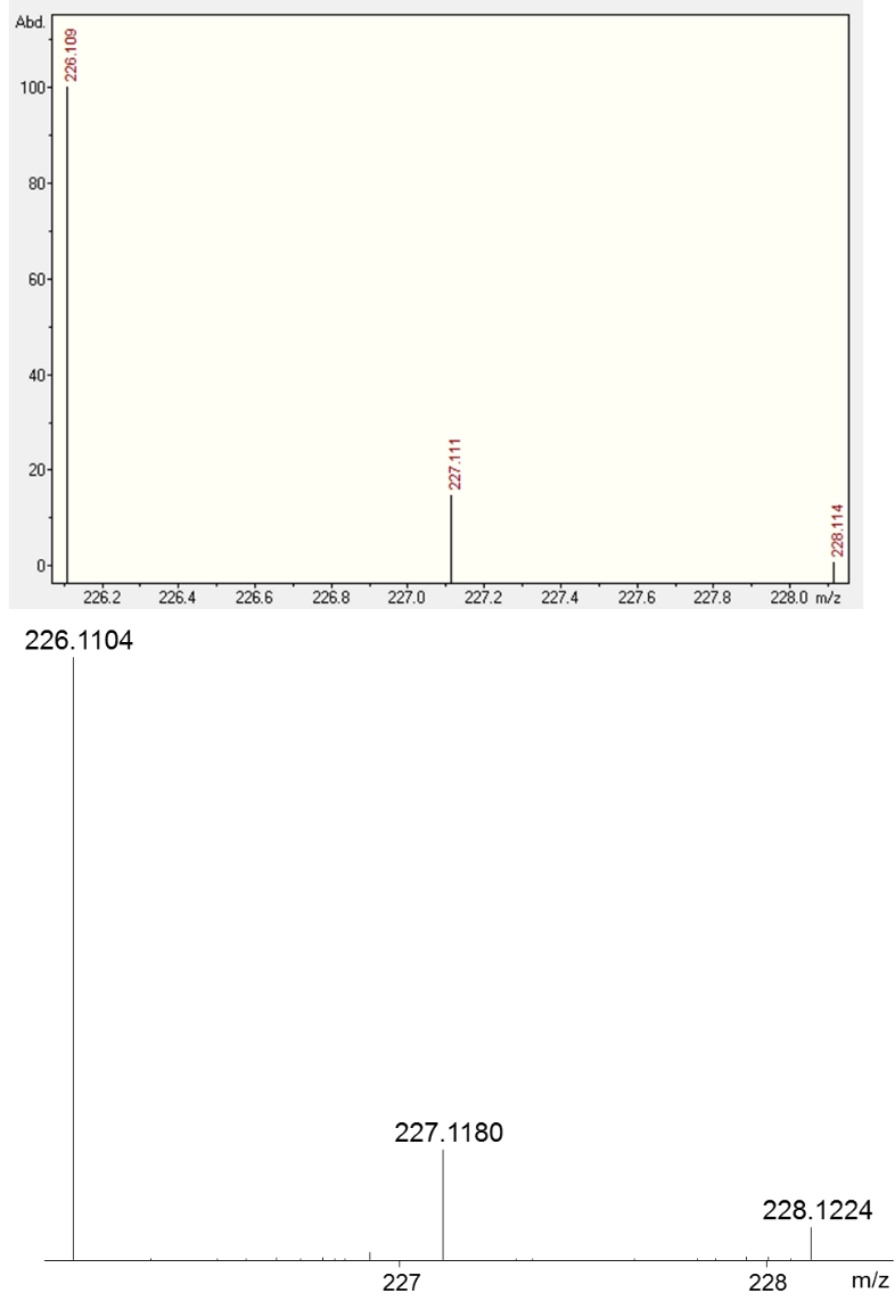

Figure S17: High-resolution ESI mass spectra of 2. Top: calculated spectrum. Bottom: experimental spectrum. 


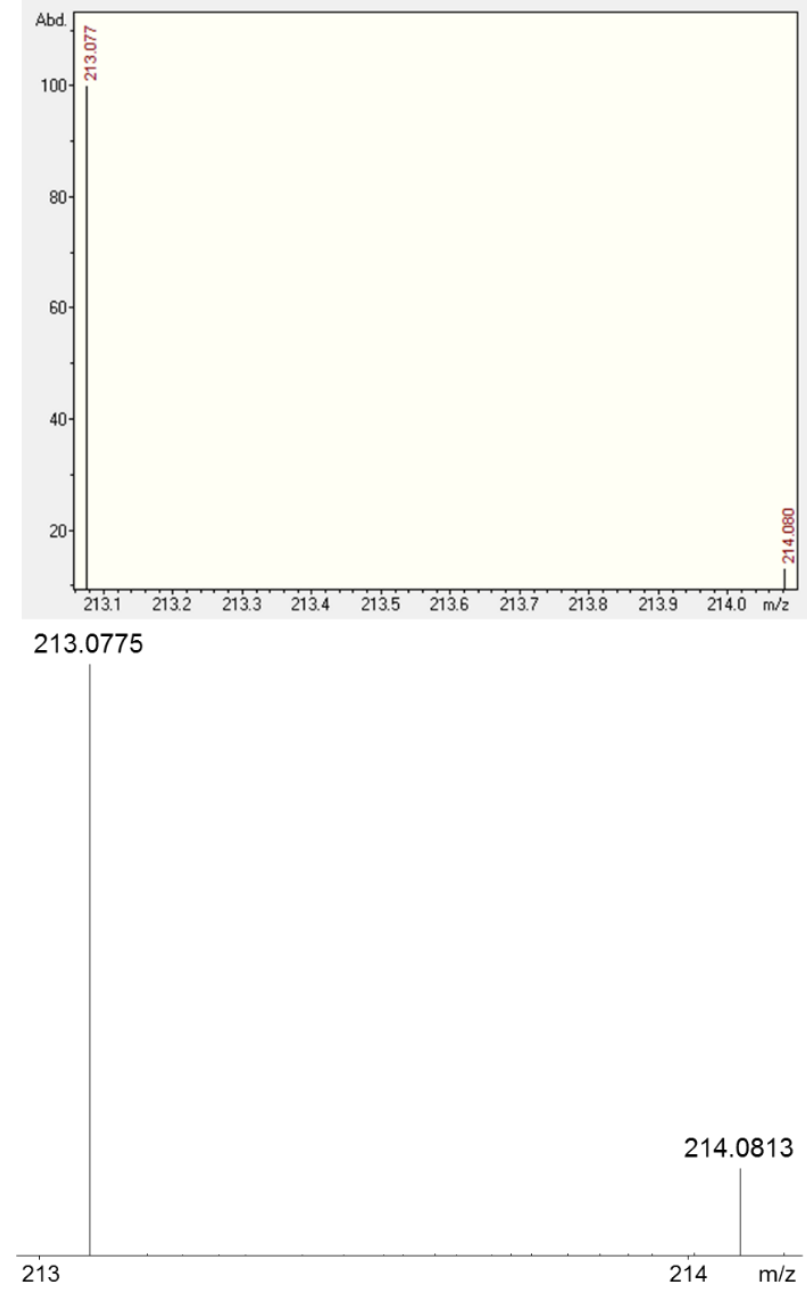

Figure S18: High-resolution ESI mass spectra of 3. Top: calculated spectrum. Bottom: experimental spectrum. 

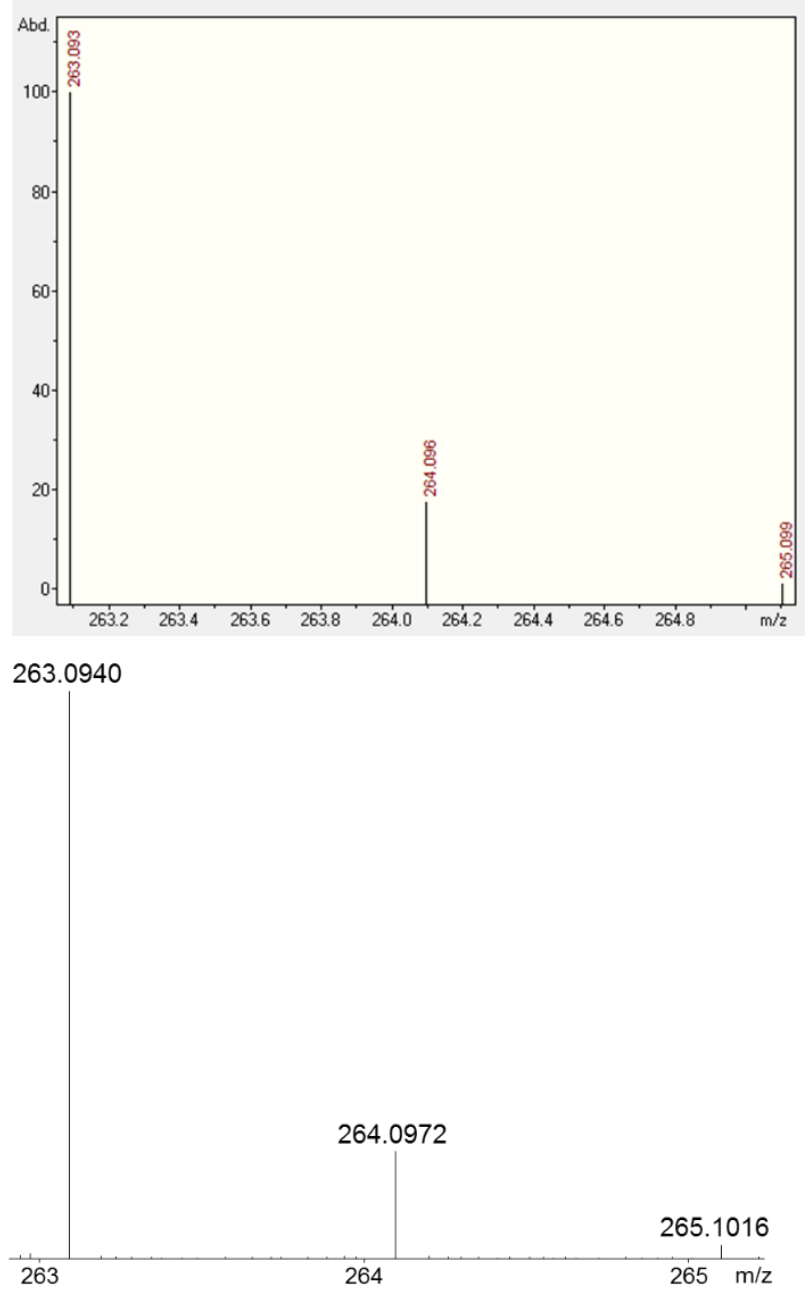

Figure S19: High-resolution ESI mass spectra of 4. Top: calculated spectrum. Bottom: experimental spectrum. 

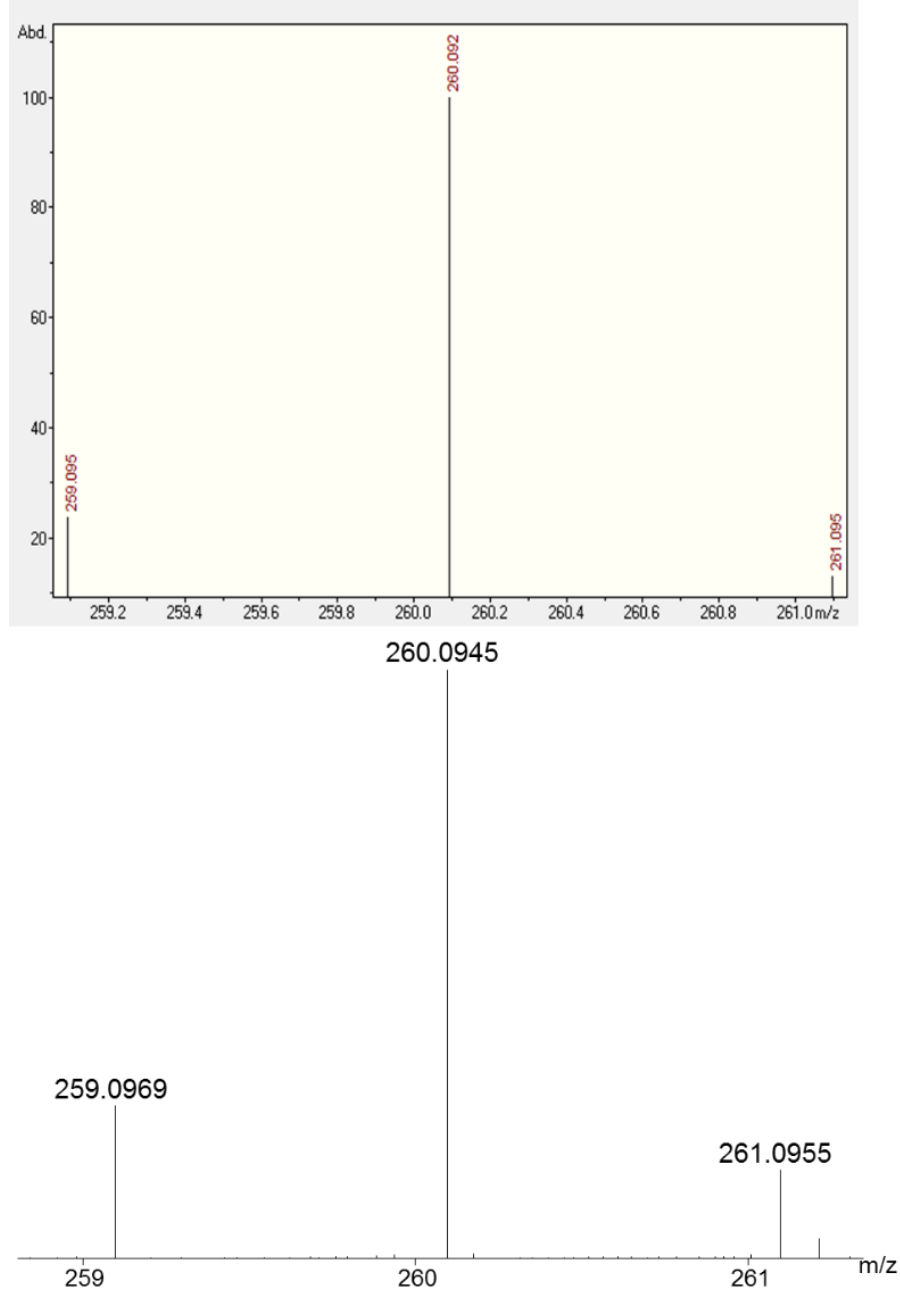

Figure S20: High-resolution ESI mass spectra of 1BF2. Top: calculated spectrum. Bottom: experimental spectrum. 

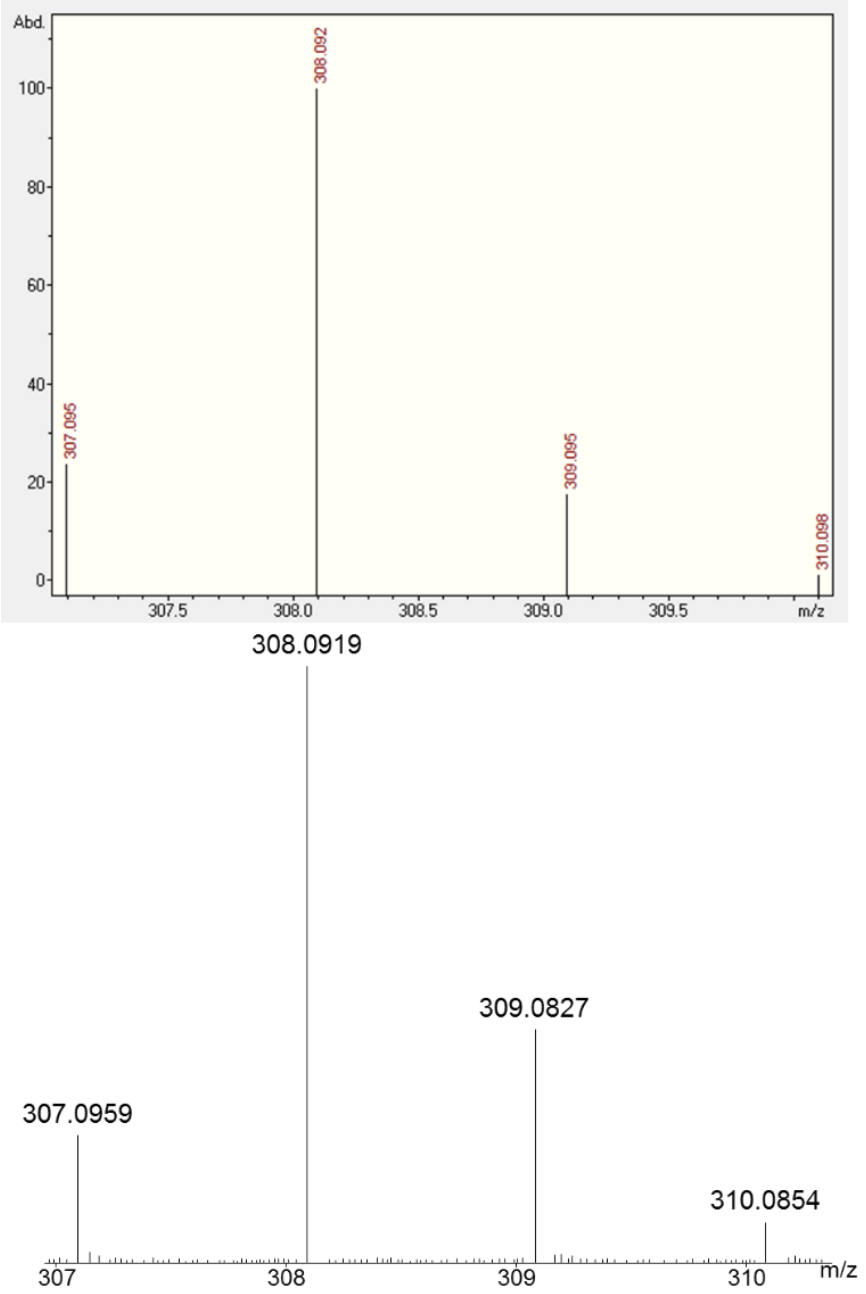

Figure S21: High-resolution ESI mass spectra of 2BF2. Top: calculated spectrum. Bottom: experimental spectrum. 

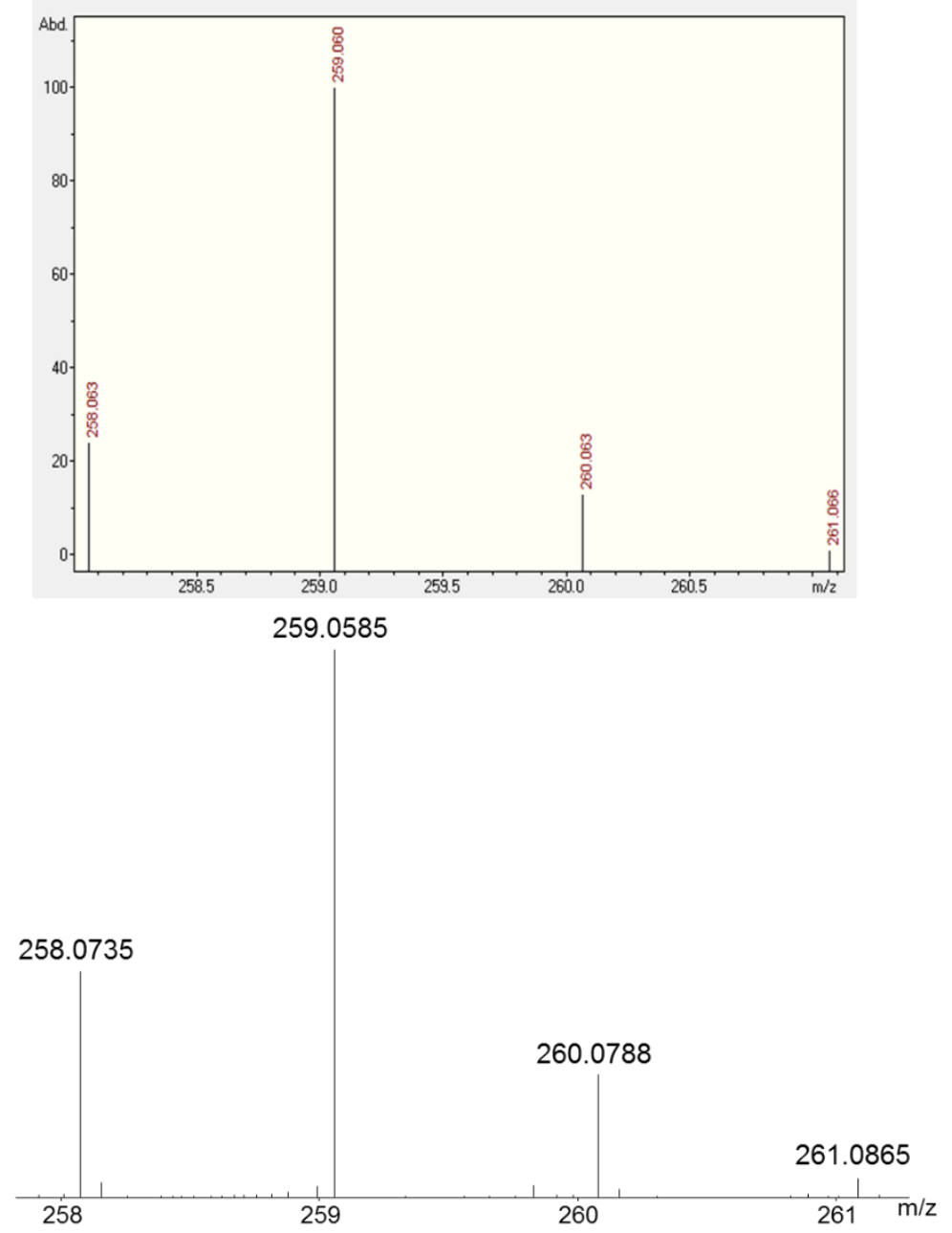

Figure S22: High-resolution ESI mass spectra of $\mathbf{3 B F}$. Top: calculated spectrum. Bottom: experimental spectrum. 

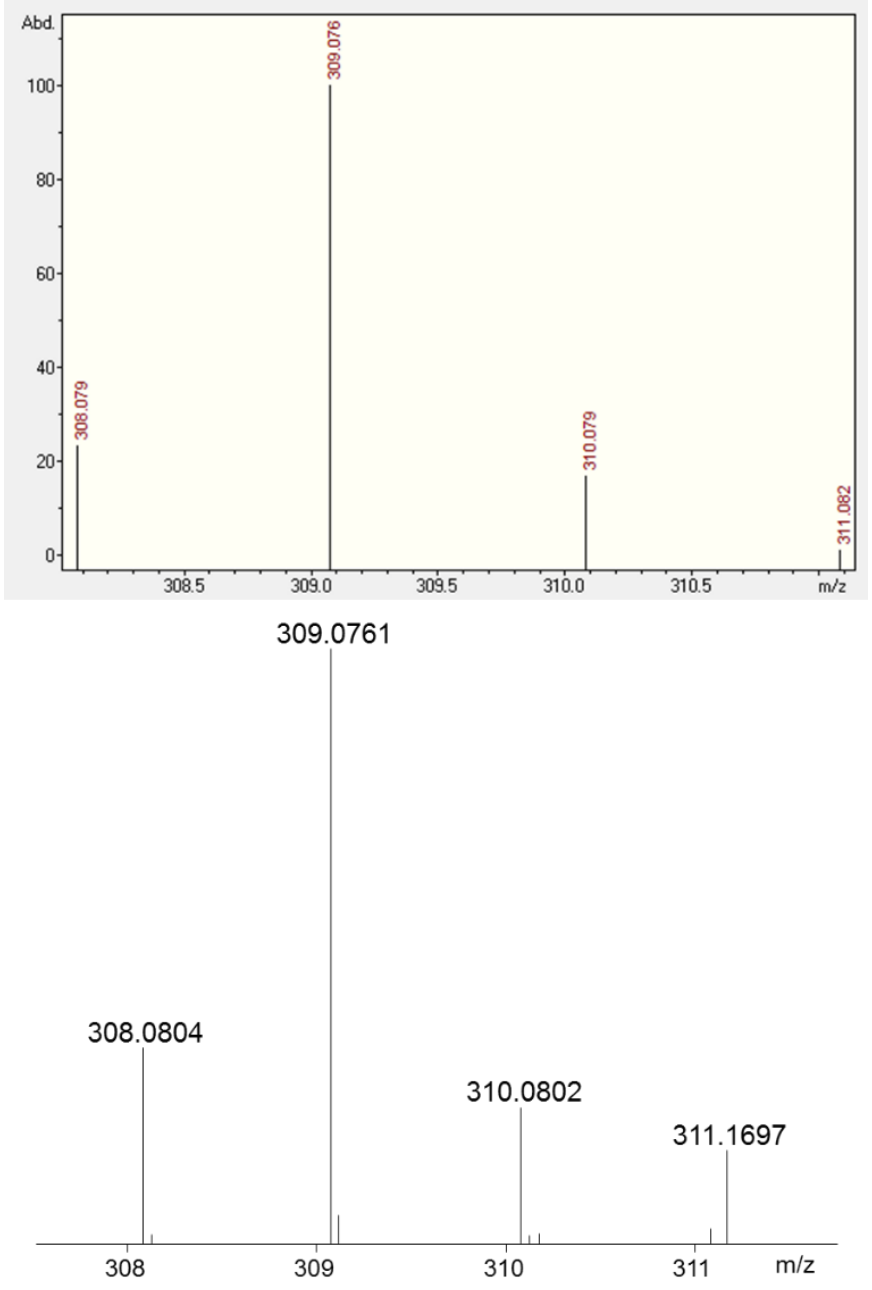

Figure S23: High-resolution ESI mass spectra of $\mathbf{4 B F}$. Top: calculated spectrum. Bottom: experimental spectrum. 


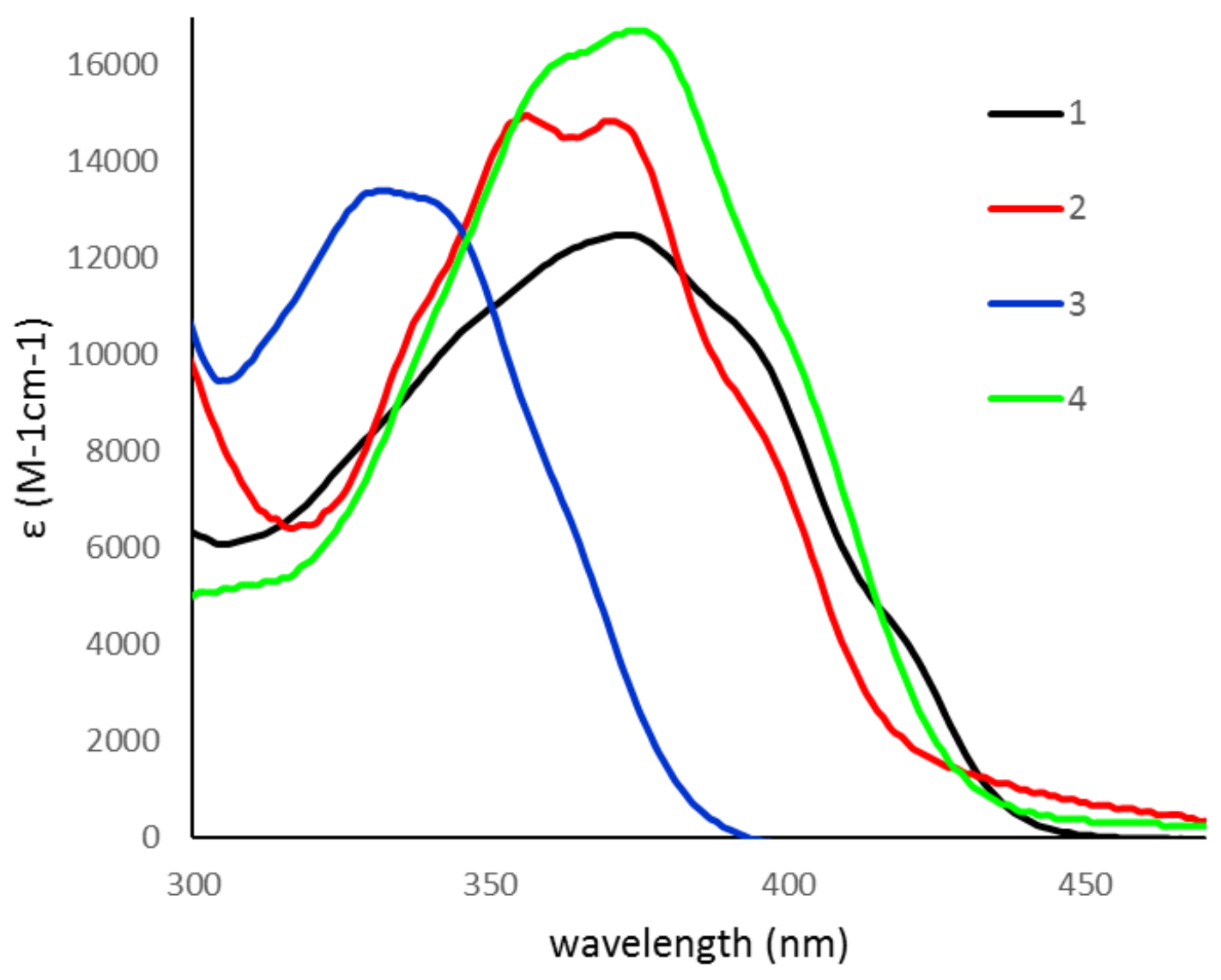

\begin{tabular}{|c|c|c|}
\hline Compound & $\lambda_{\max }(\mathrm{nm})$ & $\varepsilon 10^{-4}\left(\mathrm{M}^{-1} \mathrm{~cm}^{-1}\right)$ \\
\hline $\mathbf{1}$ & 373 & 1.25 \\
\hline $\mathbf{2}$ & 356 & 1.50 \\
\hline $\mathbf{3}$ & 332 & 1.34 \\
\hline $\mathbf{4}$ & 375 & 1.67 \\
\hline
\end{tabular}

Figure S24: UV-visible spectra for compounds 1-4 in DMF. The figure includes molar absorptivity coefficients $(\varepsilon)$, and $\lambda_{\max }(\mathrm{nm})$. 


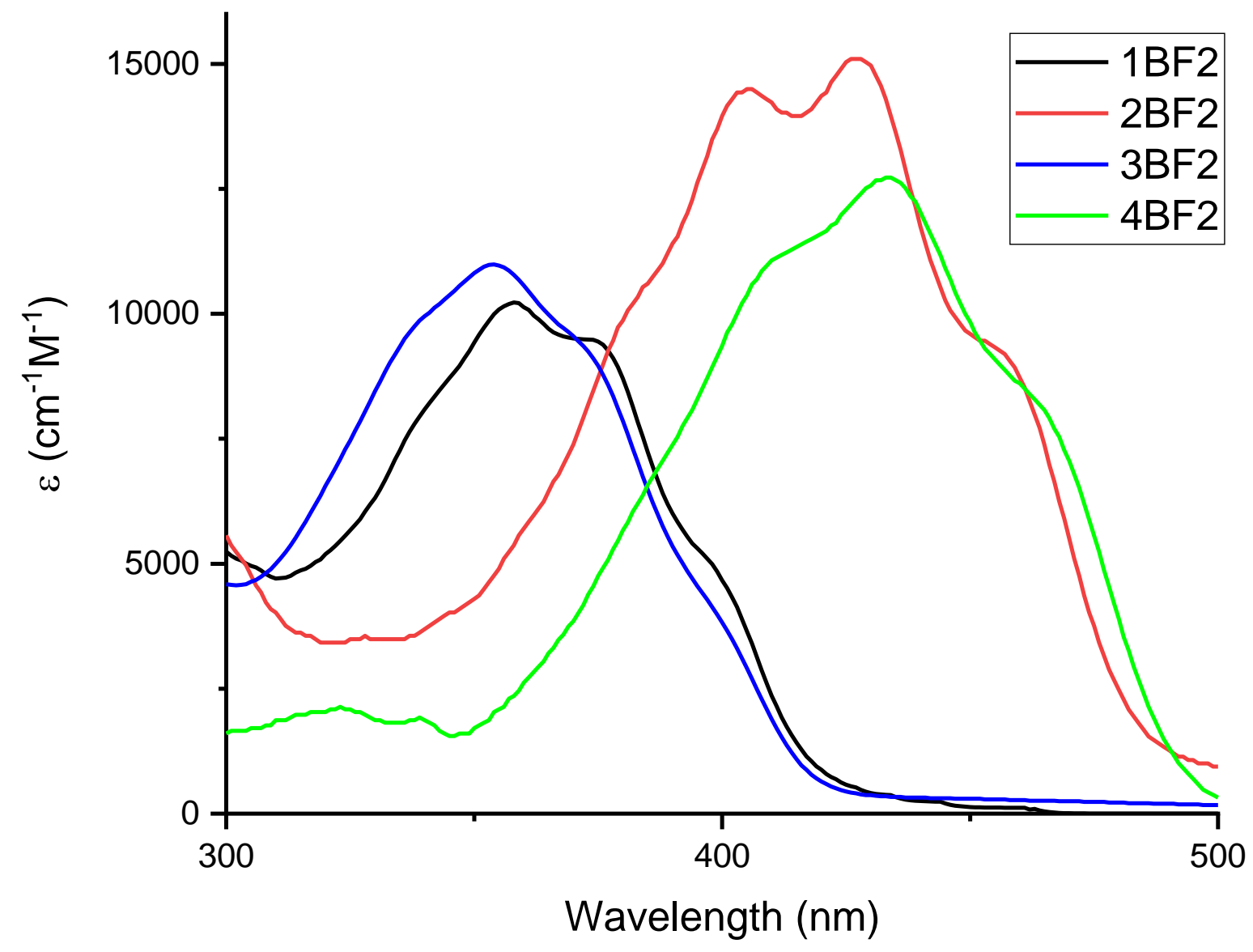

\begin{tabular}{|c|c|c|}
\hline Compound & $\lambda_{\max }(\mathrm{nm})$ & $\varepsilon 10^{-4}\left(\mathrm{M}^{-1} \mathrm{~cm}^{-1}\right)$ \\
\hline $\mathbf{1 B F}_{\mathbf{2}}$ & 358 & 1.02 \\
\hline $\mathbf{2 B F}_{\mathbf{2}}$ & 430 & 1.50 \\
\hline $\mathbf{3 B F}_{\mathbf{2}}$ & 355 & 1.10 \\
\hline $\mathbf{4 B F}_{\mathbf{2}}$ & 436 & 1.26 \\
\hline
\end{tabular}

Figure S25: UV-visible spectra for compounds $\mathbf{1 B F}_{\mathbf{2}}-\mathbf{4 B F} \mathbf{2}$ in $\mathrm{DMF}$. The figure includes molar absorptivity coefficients $(\varepsilon)$, and $\lambda_{\max }(\mathrm{nm})$. 

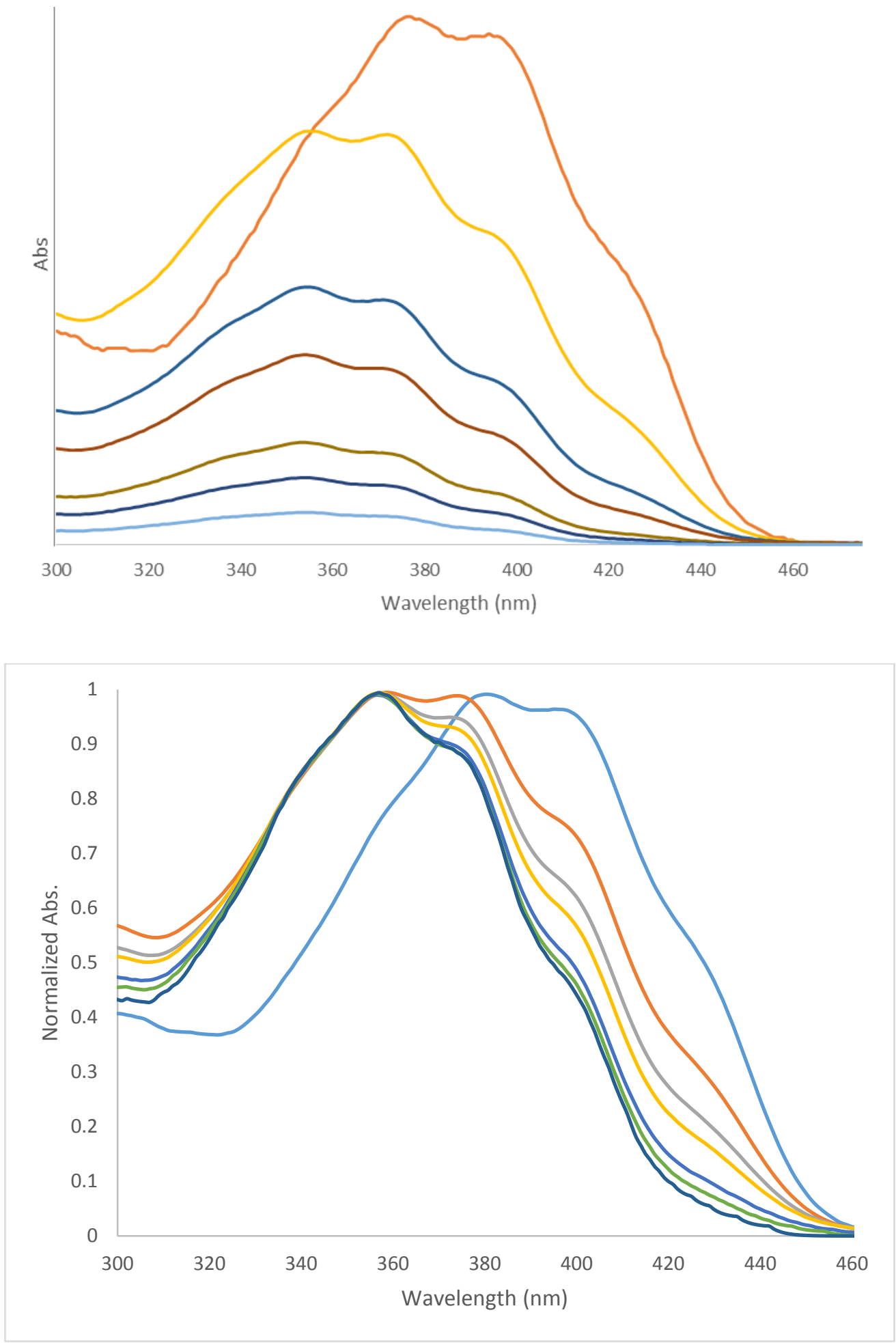

Figure S26: UV-Vis absorption titration with increasing amounts of $\mathbf{1 B F} 2$ in DMF (top), and normalized absorption spectra (bottom). 

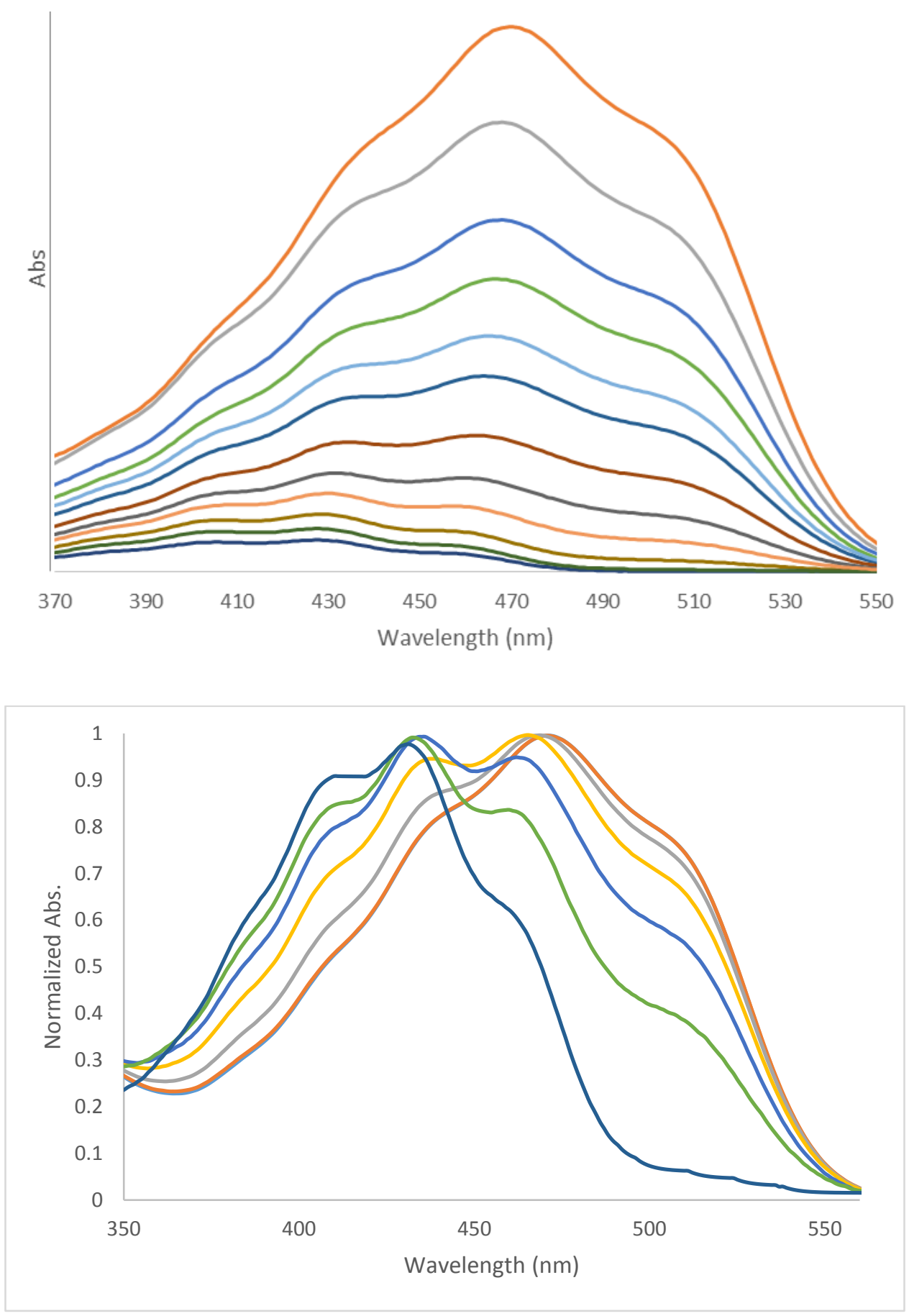

Figure S27: UV-Vis absorption titration with increasing amounts of $\mathbf{2 B}$ 2 2 in DMF (top), and normalized absorption spectra (bottom). 

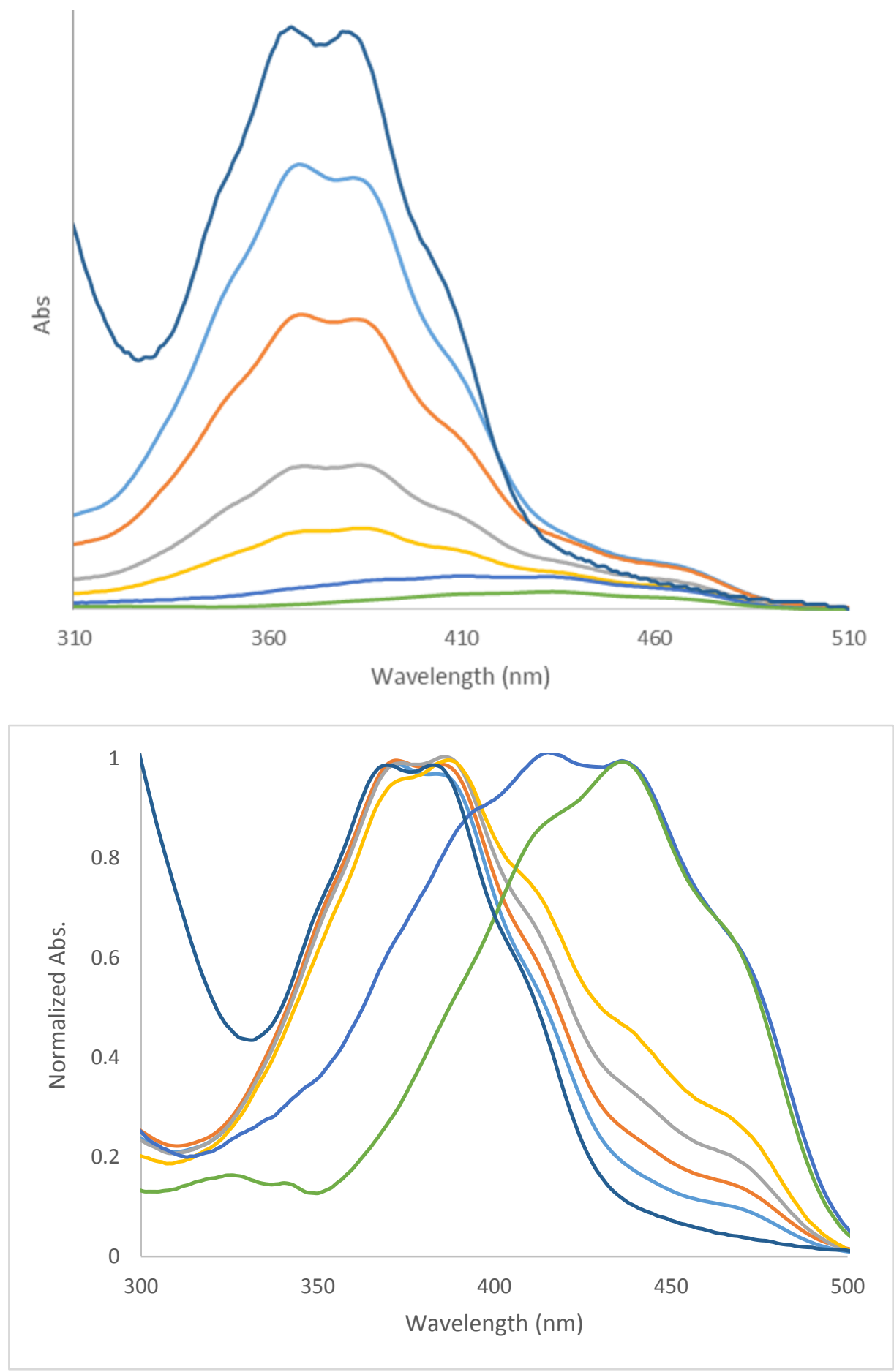

Figure S28: UV-Vis absorption titration with increasing amounts of $\mathbf{4} \mathbf{B F}_{\mathbf{2}}$ in DMF (top), and normalized absorption spectra (bottom). 

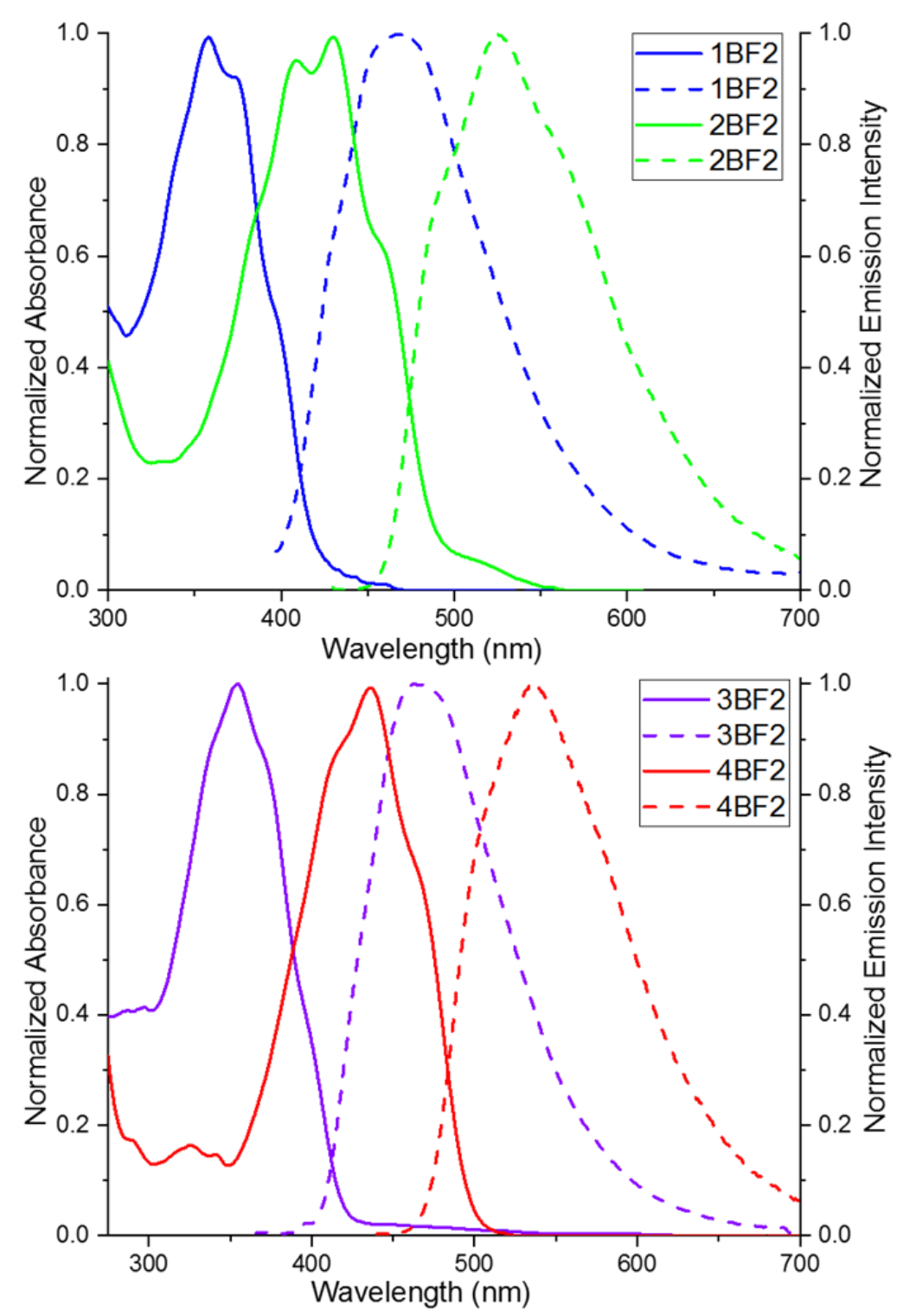

Figure S29: The normalized absorption (solid) and emission (dashed) spectra for compound $\mathbf{B B F}_{\mathbf{2}}-\mathbf{4} \mathrm{BF}_{2}$ in DMF. 


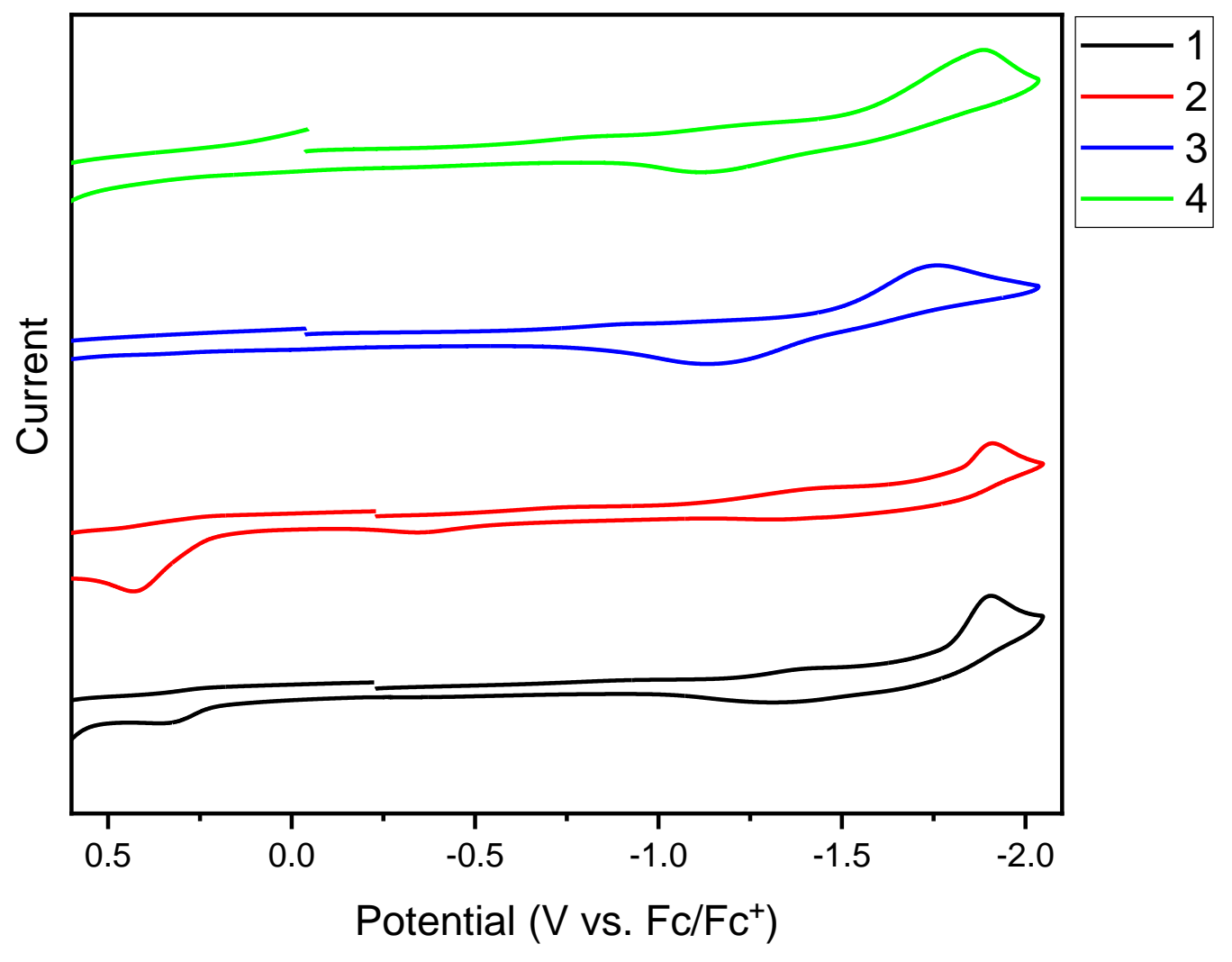

Figure S30: Cyclic voltammograms of 1-4 in DMF/0.1 $\mathrm{TBAPF}_{6}$. 


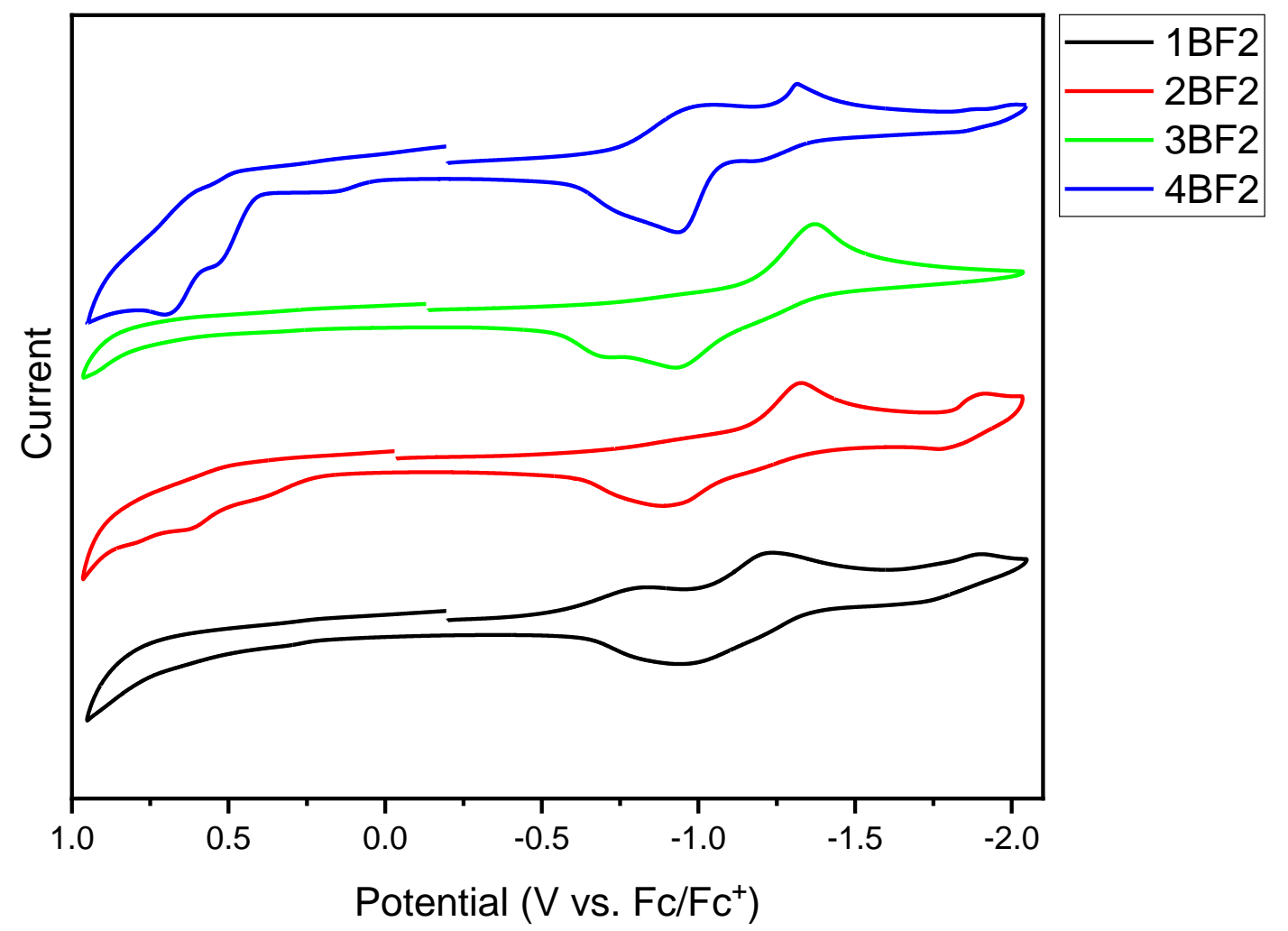

Figure S31: Cyclic voltammograms of $\mathbf{1 B F}_{\mathbf{2}} \mathbf{- 4} \mathbf{B F}_{\mathbf{2}}$ in $\mathrm{DMF} / 0.1 \mathrm{TBAPF}$. 


\section{Compound}
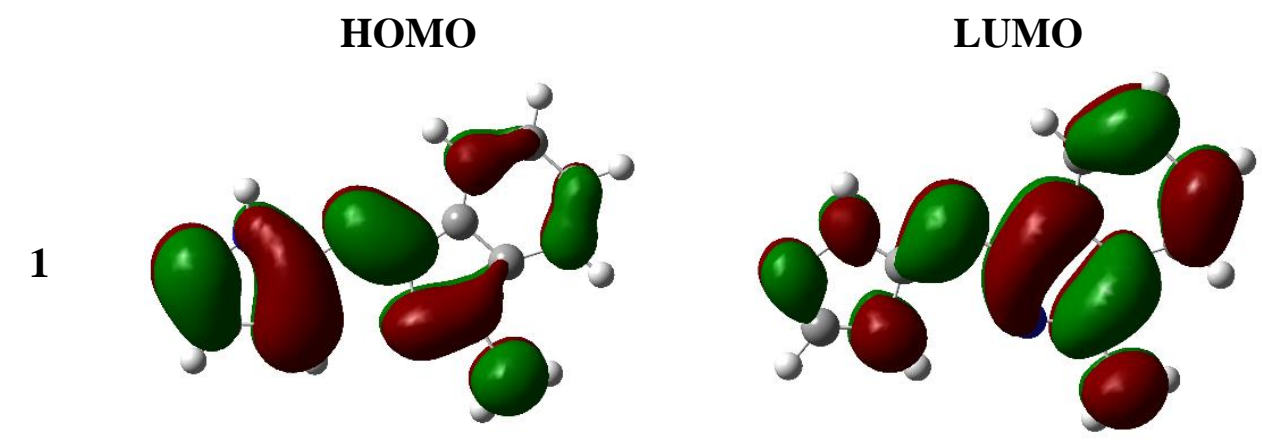

2
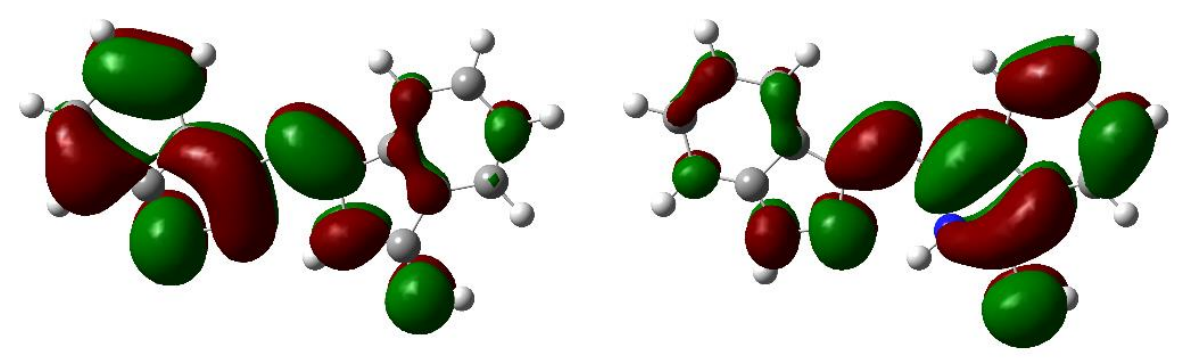

3
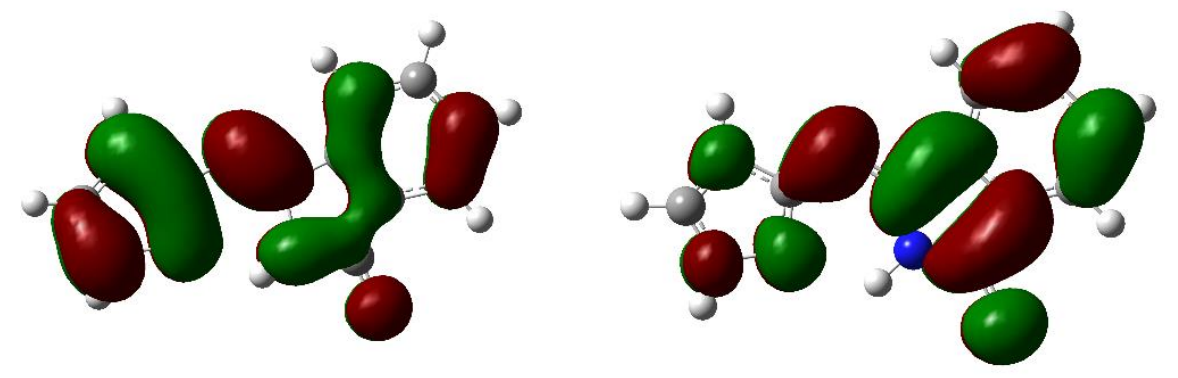

4
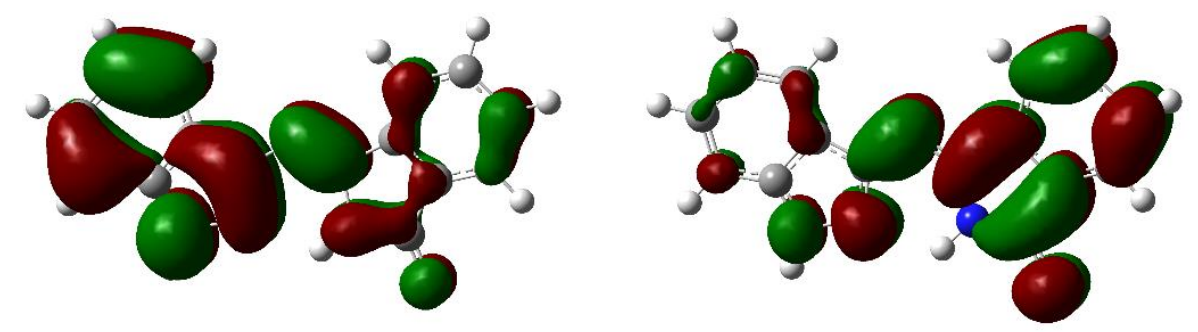

Figure S32: DFT-predicted frontier orbitals for compounds 1-4. 


\section{Compound}

HOMO

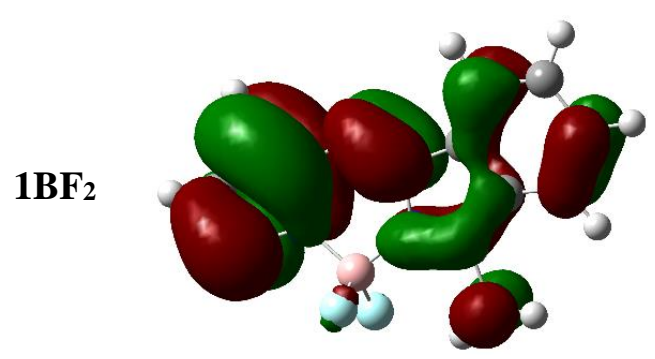

$2 \mathrm{BF}_{2}$

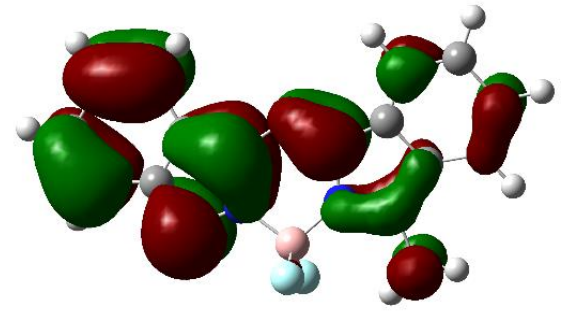

$3 \mathrm{BF}_{2}$

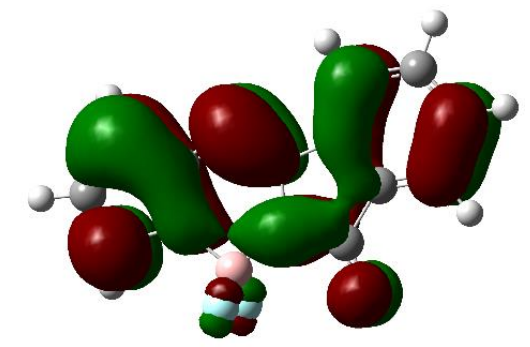

$4 \mathrm{BF}_{2}$
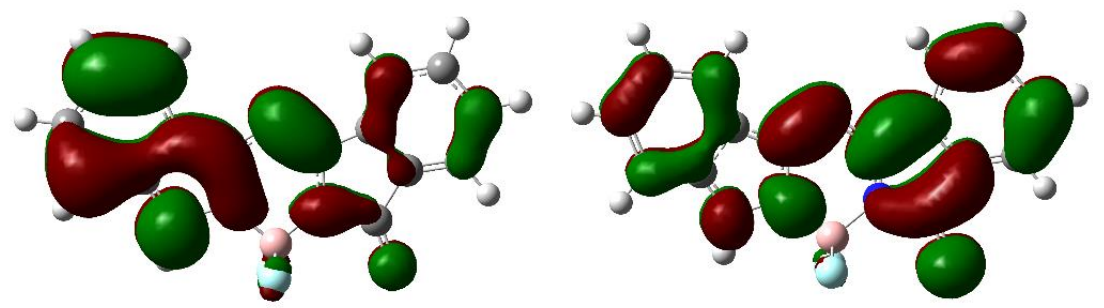

Figure S33: DFT-predicted frontier orbitals for compounds $\mathbf{1 B F} \mathbf{- 4} \mathbf{4} \mathbf{B F}_{2}$. 

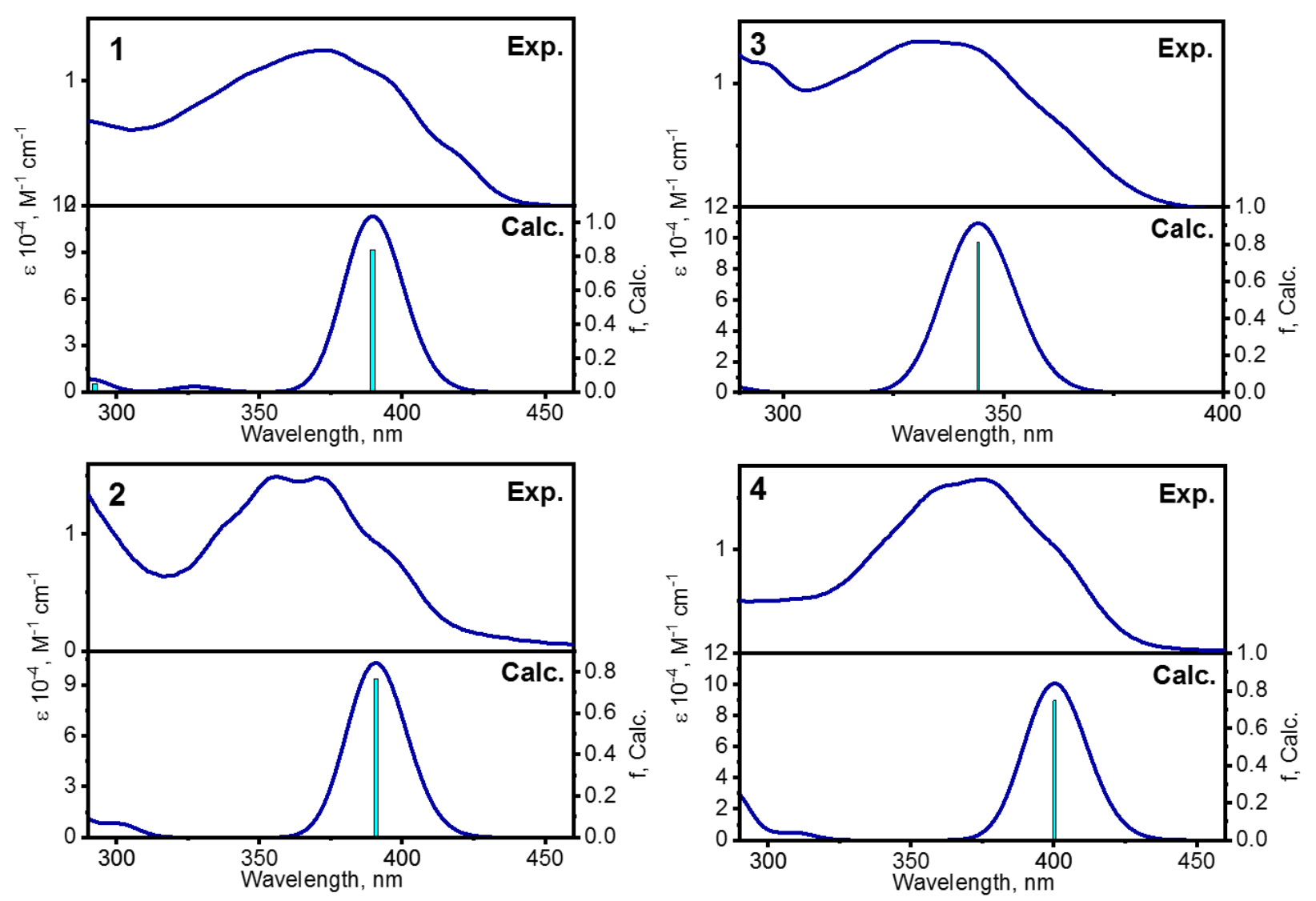

Figure S34: Experimental and B3LYP TDDFT-predicted spectra for compounds 1-4. 

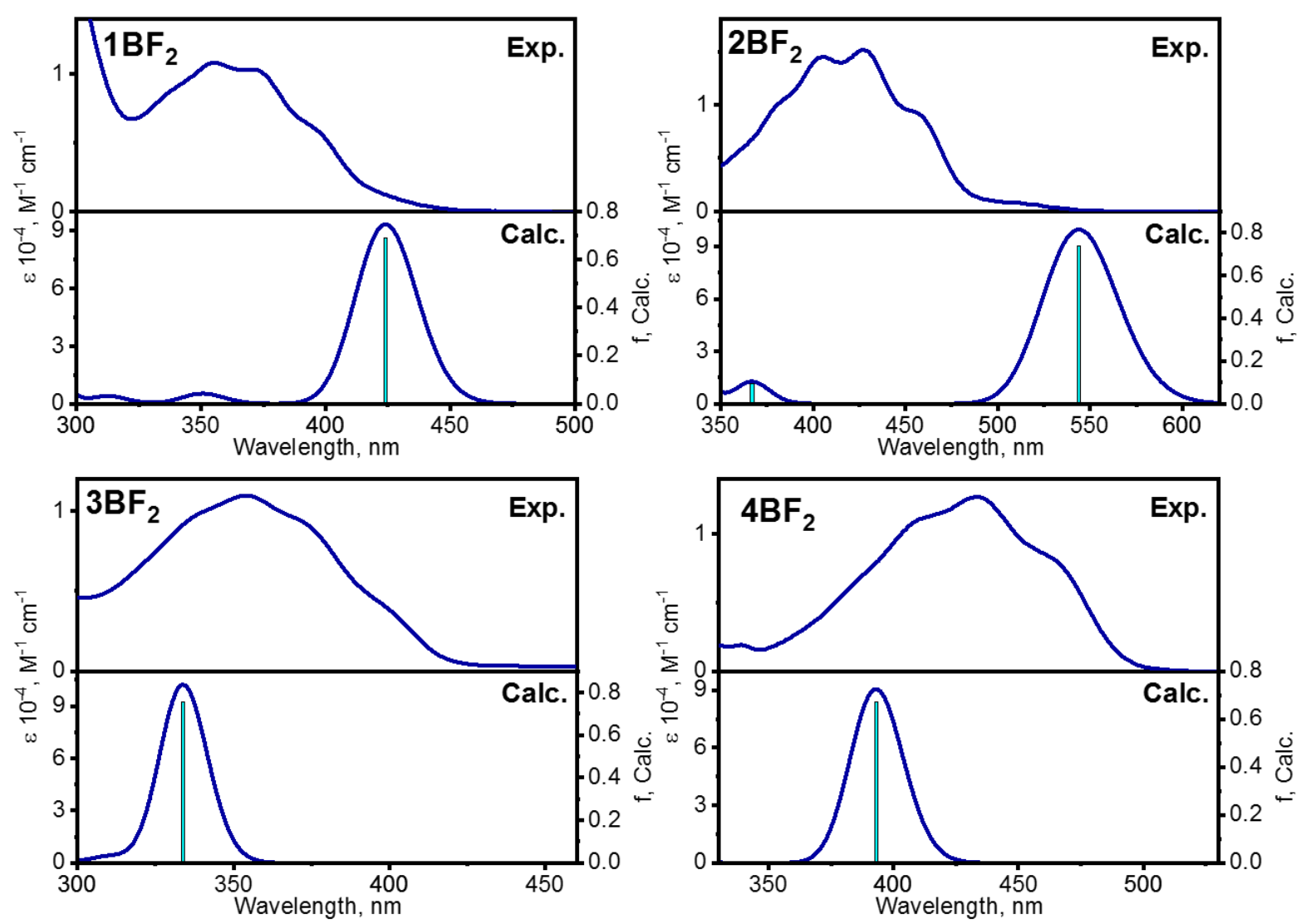

Figure S35: Experimental and B3LYP TDDFT-predicted spectra for compounds $\mathbf{1 B F}_{\mathbf{2}}-\mathbf{4} \mathbf{B F}_{2}$. 


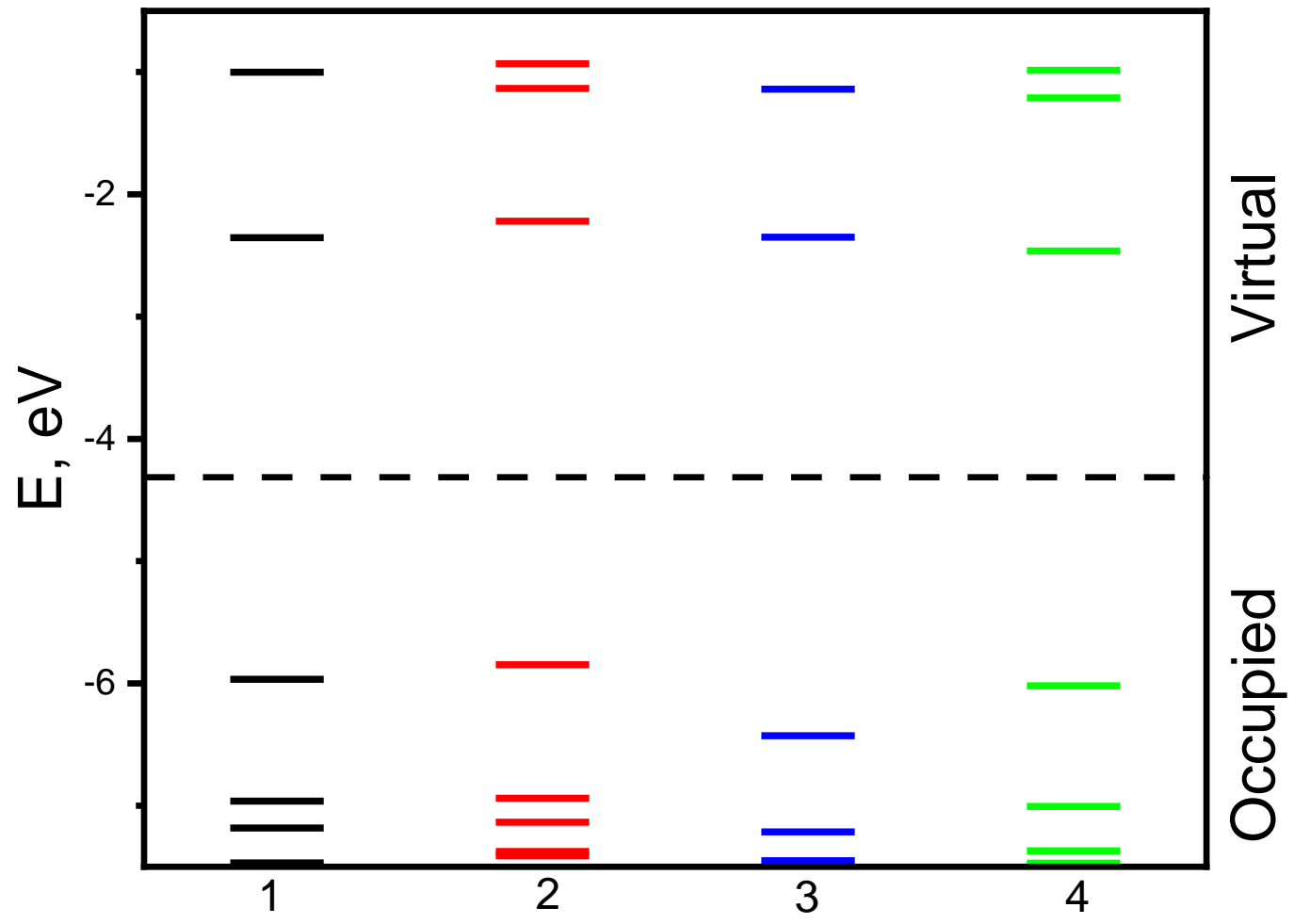

Figure S36: B3LYP relative energies of the frontier orbitals for compounds 1-4. 


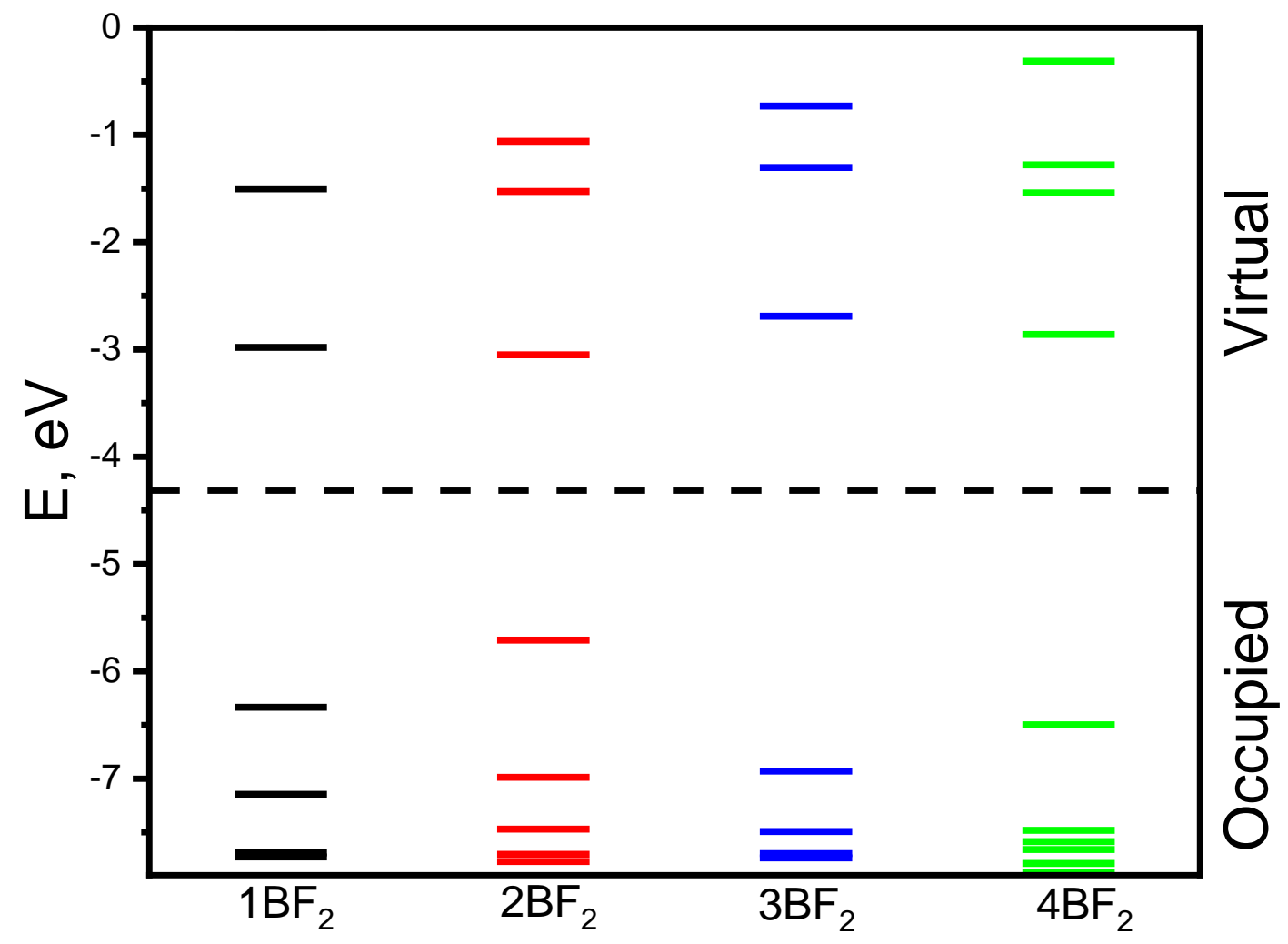

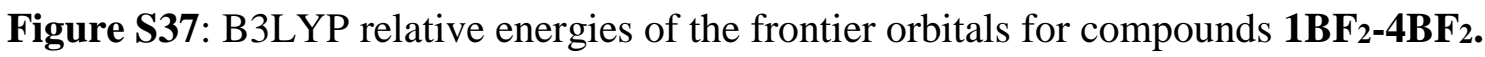




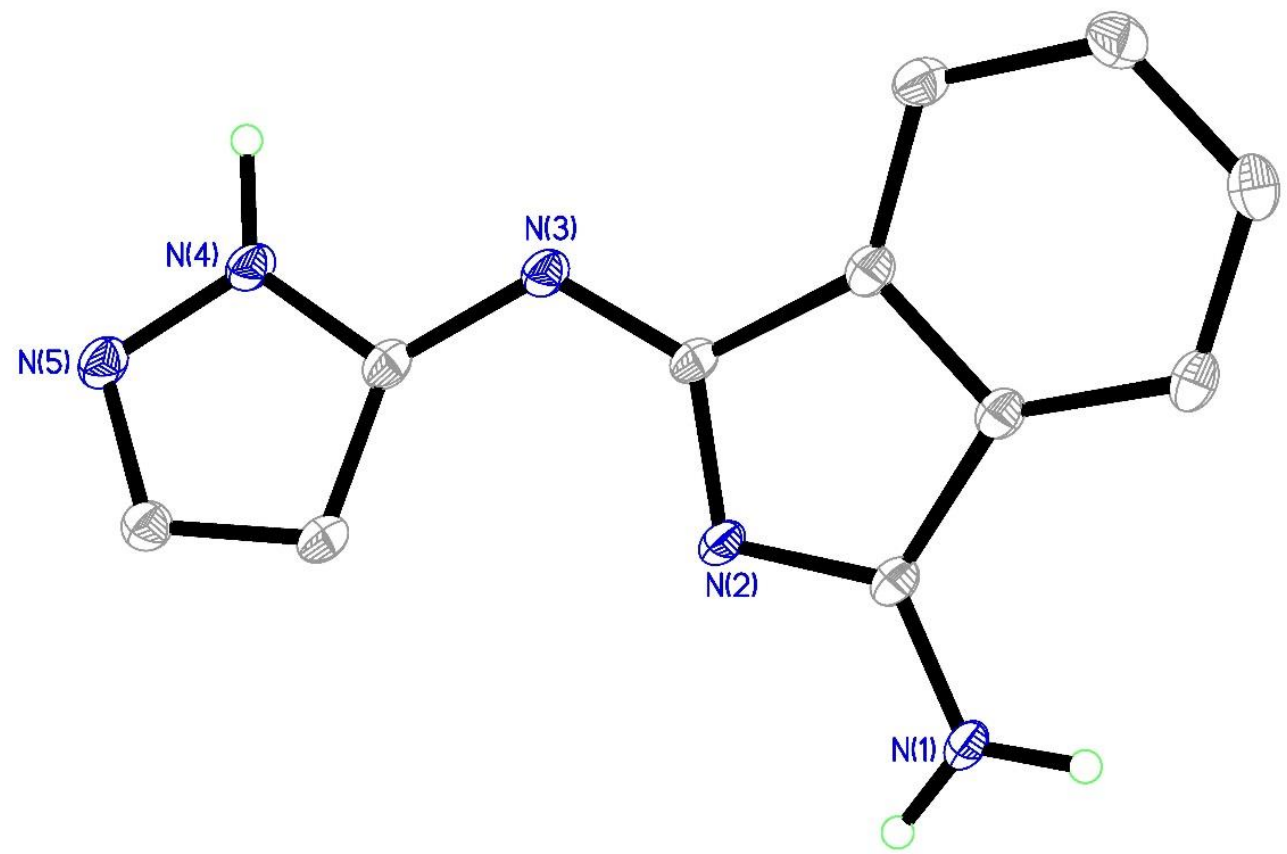

Figure S38: Structure of compound 1, showing 35\% probability ellipsoids. Hydrogen atoms except on nitrogen atoms have been omitted for clarity. 


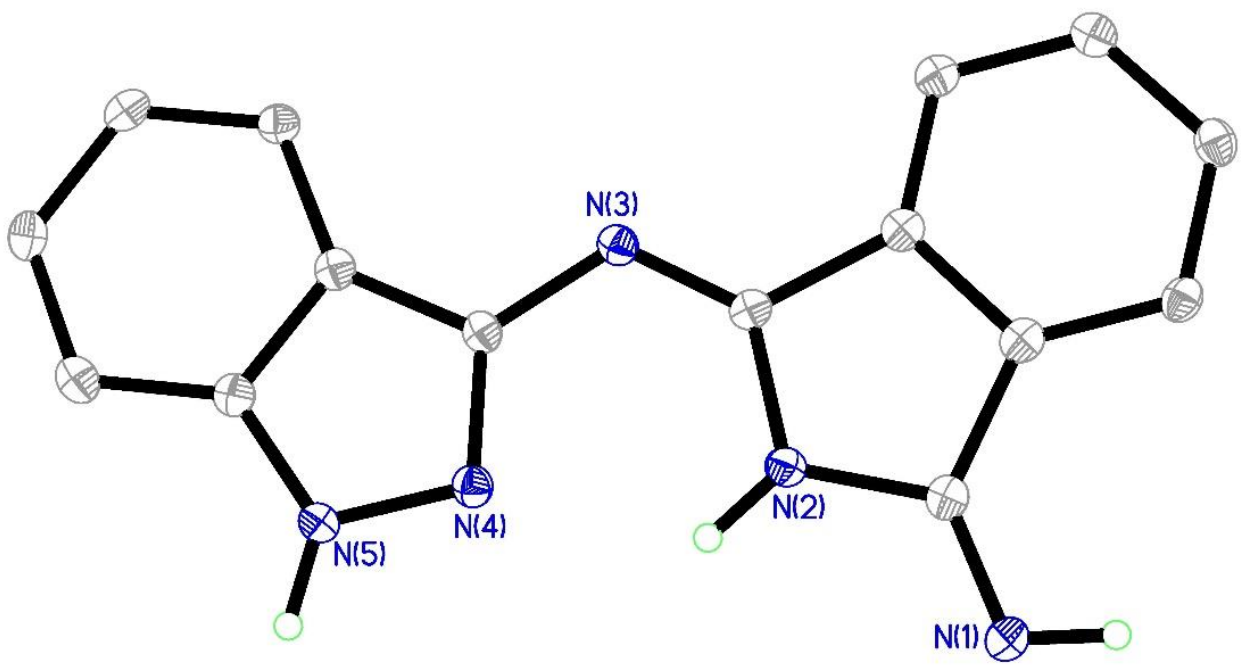

Figure S39: Structure of compound 2, showing 35\% probability ellipsoids. Hydrogen atoms except on nitrogen atoms have been omitted for clarity. 


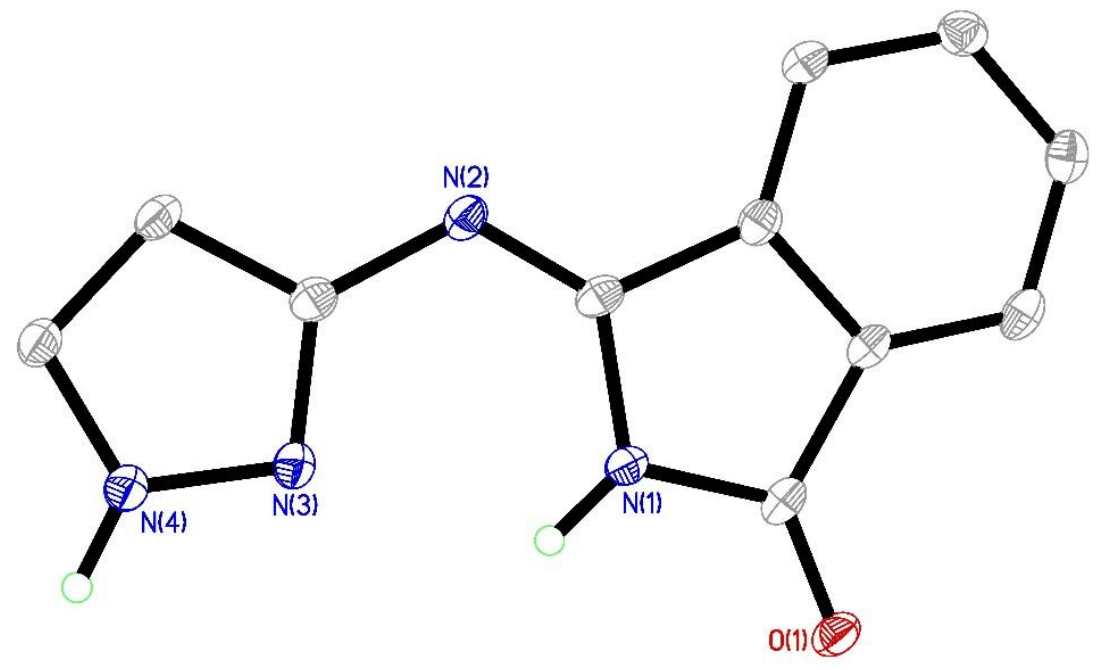

Figure S40: Structure of compound 3, showing 35\% probability ellipsoids. Hydrogen atoms except on nitrogen atoms have been omitted for clarity. 


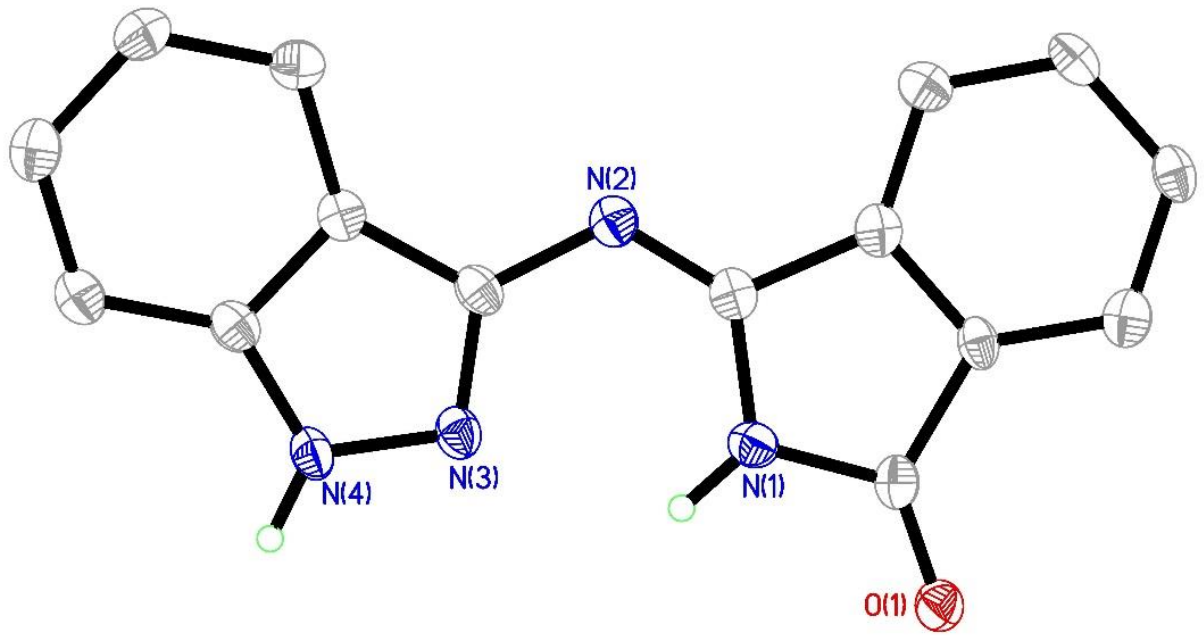

Figure S41: Structure of compound 4, showing 35\% probability ellipsoids. Hydrogen atoms except on nitrogen atoms have been omitted for clarity. 


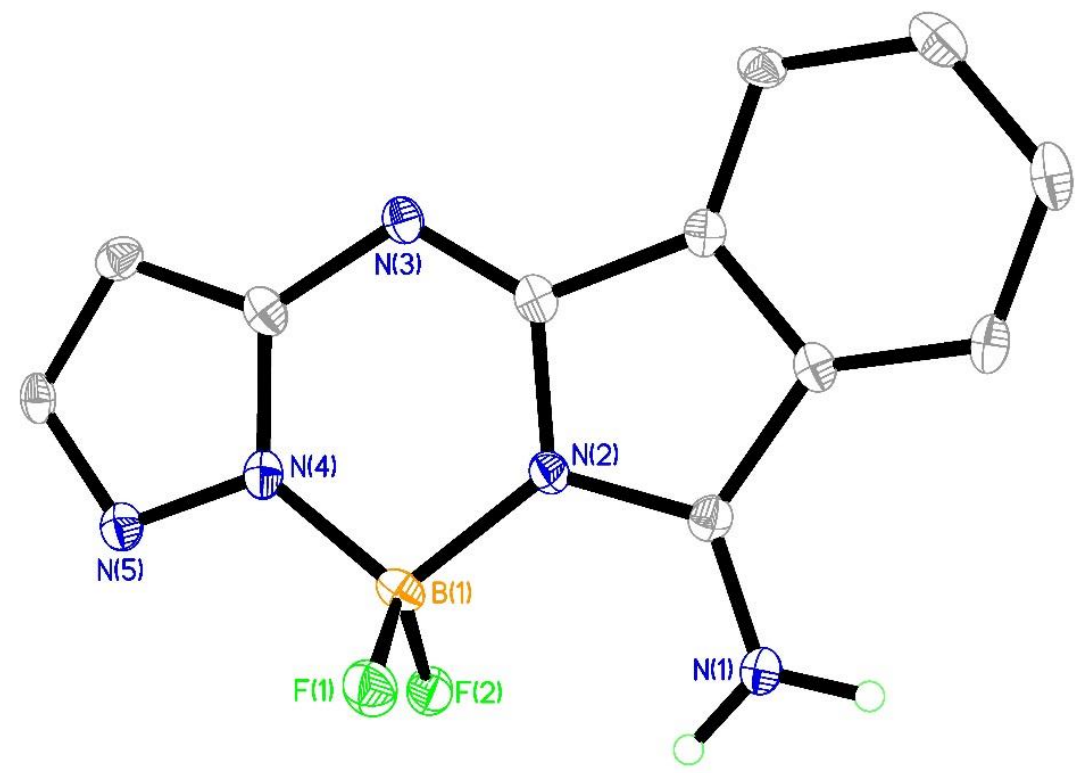

Figure S42: Structure of compound $\mathbf{1 B F}$, showing 35\% probability ellipsoids. Hydrogen atoms except on nitrogen atoms have been omitted for clarity. 


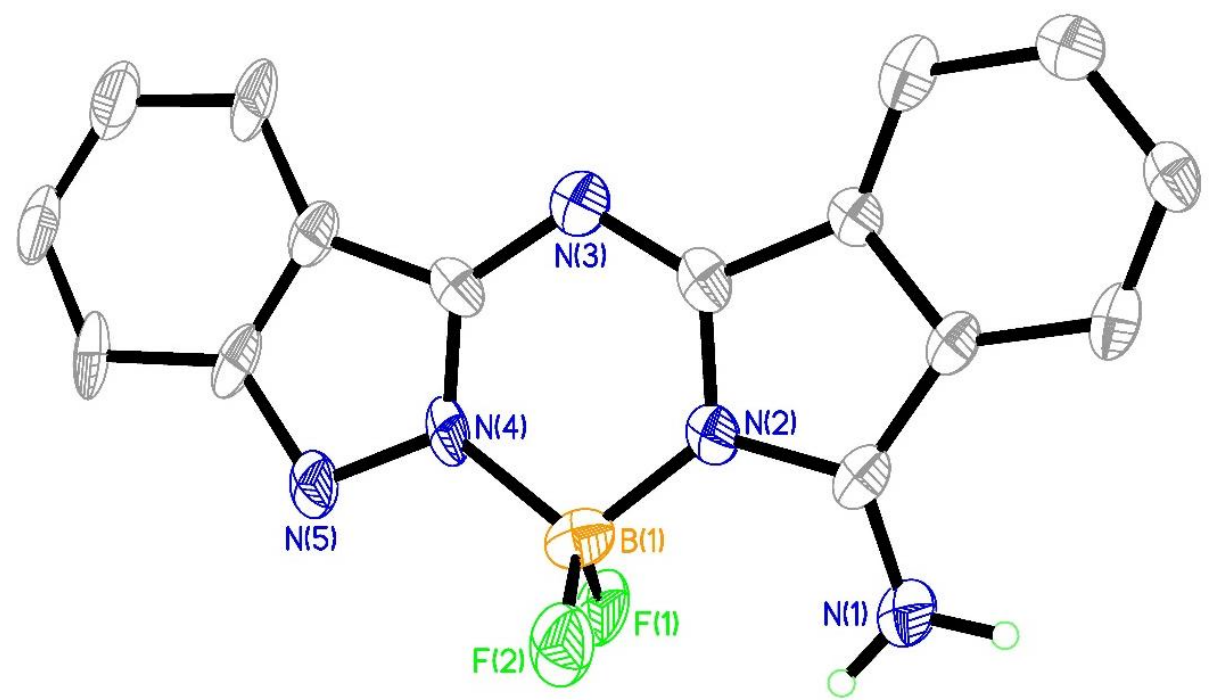

Figure S43: Structure of compound $\mathbf{2} \mathbf{B F}_{2}$, showing 35\% probability ellipsoids. Hydrogen atoms except on nitrogen atoms have been omitted for clarity. 


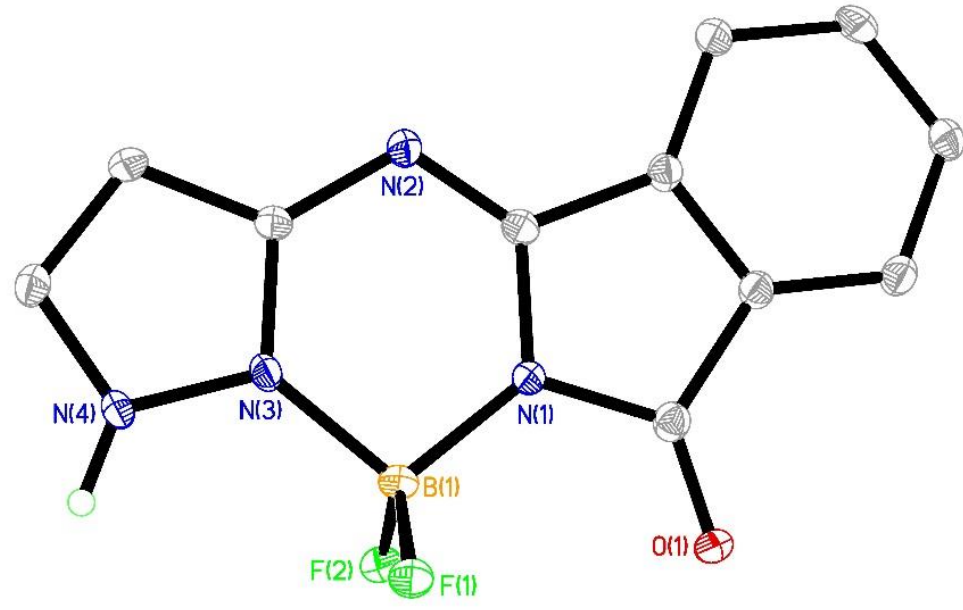

Figure S44: Structure of compound $\mathbf{3 B F}$, showing $35 \%$ probability ellipsoids. Hydrogen atoms except on nitrogen atoms have been omitted for clarity. 


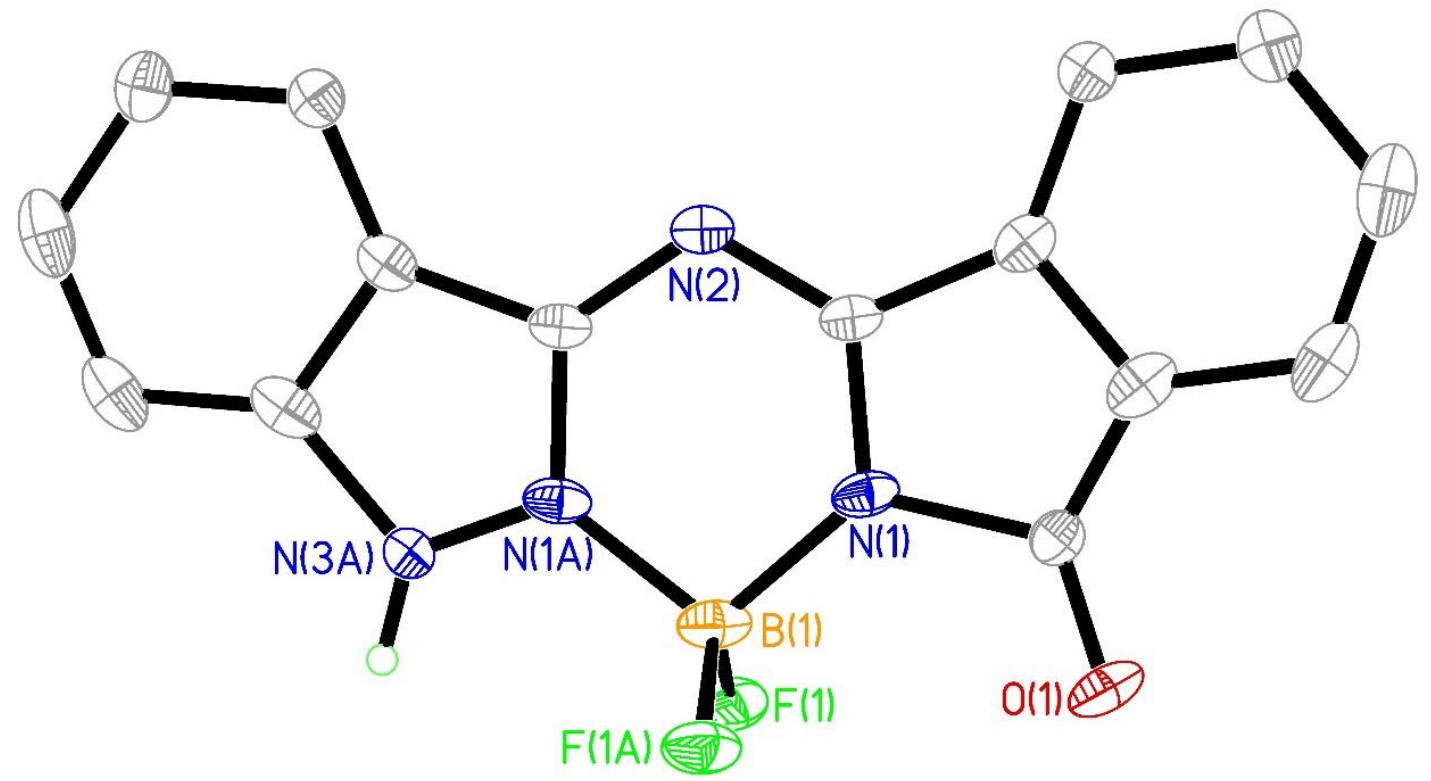

Figure S45: Structure of compound $\mathbf{4 B F}$, showing 35\% probability ellipsoids. Hydrogen atoms except on nitrogen atoms have been omitted for clarity. 
Table S1: X-ray crystal data and structure parameters for compounds 1-4.

\begin{tabular}{ccccc}
\hline Compound & $\mathbf{1}$ & $\mathbf{2}$ & $\mathbf{3}$ & $\mathbf{4}$ \\
\hline CCDC & 1988807 & 2076513 & 2076514 & 2076515 \\
\hline Empirical formula & $\mathrm{C}_{11} \mathrm{H}_{9} \mathrm{~N}_{5}$ & $\mathrm{C}_{15} \mathrm{H}_{11} \mathrm{~N}_{5}$ & $\mathrm{C}_{11} \mathrm{H}_{8} \mathrm{~N}_{4} \mathrm{O}$ & $\mathrm{C}_{15} \mathrm{H}_{10} \mathrm{~N}_{4} \mathrm{O}$ \\
\hline Formula weight & 211.23 & 261.29 & 212.21 & 262.27 \\
\hline Crystal system & Monoclinic & Monoclinic & Monoclinic & Orthorhombic \\
\hline Space group & $\mathrm{C} 2 / \mathrm{c}$ & $\mathrm{P} 21$ & $\mathrm{P} 21 / \mathrm{n}$ & Pbca \\
\hline $\mathrm{a} / \AA$ & $11.6141(5)$ & $4.83880(10)$ & $4.8287(12)$ & $13.575(3)$ \\
\hline $\mathrm{b} / \AA$ & $12.7038(5)$ & $9.0472(3)$ & $15.837(4)$ & $7.2883(11)$ \\
\hline $\mathrm{c} / \AA$ & $13.4922(6)$ & $13.6010(4)$ & $12.672(3)$ & $24.506(4)$ \\
\hline$\alpha\left(^{\circ}\right)$ & 90 & 90 & 90 & 90 \\
\hline$\beta\left(^{\circ}\right)$ & $99.080(3)$ & $97.360(2)$ & $95.126(9)$ & 90 \\
\hline$\gamma\left({ }^{\circ}\right)$ & 90 & 90 & 90 & 90 \\
\hline Volume $\left(\AA^{3}\right)$ & $1965.74(15)$ & $590.51(3)$ & $965.2(4)$ & $2424.6(7)$ \\
\hline $\mathrm{Z}$ & 8 & 2 & 4 & 8 \\
\hline Dc $\left({\left.\mathrm{Mg} / \mathrm{m}^{3}\right)}^{\circ}\right.$ & 1.427 & 1.469 & 1.460 & 1.437 \\
\hline$\mu\left(\mathrm{mm}^{-1}\right)$ & 0.757 & 0.752 & 0.100 & 0.096 \\
\hline $\mathrm{F}(000)$ & 880 & 272 & 440 & 1088 \\
\hline reflns collected & 6403 & 4071 & 17414 & 35526 \\
\hline indep. reflns & 1687 & 1838 & 1713 & 2140 \\
\hline $\mathrm{GOF}$ on $\mathrm{F}^{2}$ & 1.046 & 1.032 & 1.046 & 1.038 \\
\hline $\mathrm{R} 1\left(\right.$ on $\mathrm{F}_{\mathrm{o}}^{2}, \mathrm{I}>$ & 0.0461 & 0.0259 & 0.0645 & 0.0844 \\
$2 \sigma(\mathrm{I}))$ & & & & \\
\hline wR2 (on $\mathrm{F}_{\mathrm{o}}^{2}, \mathrm{I}>$ & 0.1266 & 0.0624 & 0.1429 & 0.1691 \\
$2 \sigma(\mathrm{I}))$ & & & & \\
\hline $\mathrm{R} 1(\mathrm{all}$ data) & 0.0530 & 0.0276 & 0.0988 & 0.1762 \\
\hline wR2 (all data) & 0.1336 & 0.0634 & 0.1598 & 0.2109 \\
\hline
\end{tabular}




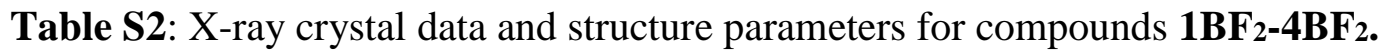

\begin{tabular}{|c|c|c|c|c|}
\hline Compound & $1 \mathrm{BF}_{2}$ & $2 \mathrm{BF}_{2}$ & $3 B_{2}$ & $4 \mathbf{B F}_{2}$ \\
\hline CCDC & 2076516 & 2076517 & 2076518 & 2052191 \\
\hline Empirical formula & $\mathrm{C}_{11} \mathrm{H}_{8} \mathrm{BF}_{2} \mathrm{~N}_{5}$ & $\mathrm{C}_{18} \mathrm{H}_{17} \mathrm{BF}_{2} \mathrm{~N}_{6} \mathrm{O}$ & $\mathrm{C}_{11} \mathrm{H}_{7} \mathrm{BF}_{2} \mathrm{~N}_{4} \mathrm{O}$ & $\mathrm{C}_{15} \mathrm{H}_{9} \mathrm{BF}_{2} \mathrm{~N}_{4} \mathrm{O}$ \\
\hline Formula weight & 259.03 & 382.18 & 260.02 & 310.07 \\
\hline Crystal system & Orthorhombic & Orthorhombic & Monoclinic & Monoclinic \\
\hline Space group & Pbca & Pbca & $\mathrm{P} 21 / \mathrm{c}$ & $\mathrm{C} 2 / \mathrm{c}$ \\
\hline $\mathrm{a} / \AA$ & $13.285(17)$ & $9.1327(14)$ & $7.0580(15)$ & $14.7987(12)$ \\
\hline $\mathrm{b} / \AA$ & $11.549(11)$ & 19.121(3) & $10.556(2)$ & $14.1332(14)$ \\
\hline $\mathrm{c} / \AA$ & $14.286(13)$ & $20.035(3)$ & $14.589(3)$ & $6.9968(7)$ \\
\hline$\alpha\left(^{\circ}\right)$ & 90 & 90 & 90 & 60 \\
\hline$\beta\left(^{\circ}\right)$ & 90 & 90 & 102.471(9) & $116.300(3)$ \\
\hline$\gamma\left({ }^{\circ}\right)$ & 90 & 90 & 90 & 90 \\
\hline Volume $\left(\AA^{3}\right)$ & 2192(4) & $3498.5(10)$ & 1061.3(4) & $1311.9(2)$ \\
\hline $\mathrm{Z}$ & 8 & 8 & 4 & 4 \\
\hline $\operatorname{Dc}\left(\mathrm{Mg} / \mathrm{m}^{3}\right)$ & 1.570 & 1.451 & 1.627 & 1.574 \\
\hline$\mu\left(\mathrm{mm}^{-1}\right)$ & 0.124 & 0.109 & 0.132 & 0.122 \\
\hline $\mathrm{F}(000)$ & 1056 & 1584 & 528 & 632 \\
\hline reflns collected & 35264 & 44672 & 19180 & 11909 \\
\hline indep. reflns & 1931 & 2914 & 1883 & 1629 \\
\hline GOF on $F^{2}$ & 1.129 & 1.095 & 1.043 & 1.225 \\
\hline $\mathrm{R} 1\left(\right.$ on $\left.\mathrm{F}_{\mathrm{o}}^{2}, \mathrm{I}>2 \sigma(\mathrm{I})\right)$ & 0.0795 & 0.1571 & 0.0540 & 0.0556 \\
\hline $\begin{array}{c}\text { wR2 }\left(\text { on F }^{2}, I>\right. \\
2 \sigma(\mathrm{I}))\end{array}$ & 0.1792 & 0.3194 & 0.1118 & 0.1206 \\
\hline R1 (all data) & 0.1112 & 0.1953 & 0.0888 & 0.0674 \\
\hline wR2 (all data) & 0.1925 & 0.3406 & 0.1245 & 0.1238 \\
\hline
\end{tabular}


Table S3: Photophysical properties of compounds $\mathbf{1 B F}-\mathbf{4 B F}$ in DMF.

\begin{tabular}{|c|c|c|c|c|c|}
\hline Compound & $\begin{array}{c}\lambda_{\text {abs }}(\mathrm{nm}) \\
\text { (Monomer) }\end{array}$ & $\begin{array}{c}\lambda_{\mathrm{em}}(\mathrm{nm}) \\
\text { (Monomer) }\end{array}$ & $\begin{array}{c}\Phi_{\mathrm{f}} \\
\text { (Monomer) }\end{array}$ & $\begin{array}{c}\lambda_{\text {abs }}(\mathrm{nm}) \\
\text { (Aggregate) }\end{array}$ & $\begin{array}{c}\lambda_{\text {em }}(\mathrm{nm}) \\
\text { (Aggregate) }\end{array}$ \\
\hline $\mathbf{1} \mathbf{B F}_{\mathbf{2}}$ & 358 & 467 & 0.01 & 378 & 490 \\
\hline $\mathbf{2} \mathbf{B F}_{\mathbf{2}}$ & 430 & 529 & 0.64 & 469 & 590 \\
\hline $\mathbf{3 B F}_{\mathbf{2}}$ & 355 & 464 & 0.04 & - & - \\
\hline $\mathbf{4} \mathbf{B F}_{\mathbf{2}}$ & 436 & 535 & 0.74 & 356 & - \\
\hline
\end{tabular}


Table S4: TDDFT-predicted energies and expansion coefficients for compound 1 (only excited states with $\mathrm{f}>0.05$ and $\lambda>270 \mathrm{~nm}$ are listed).

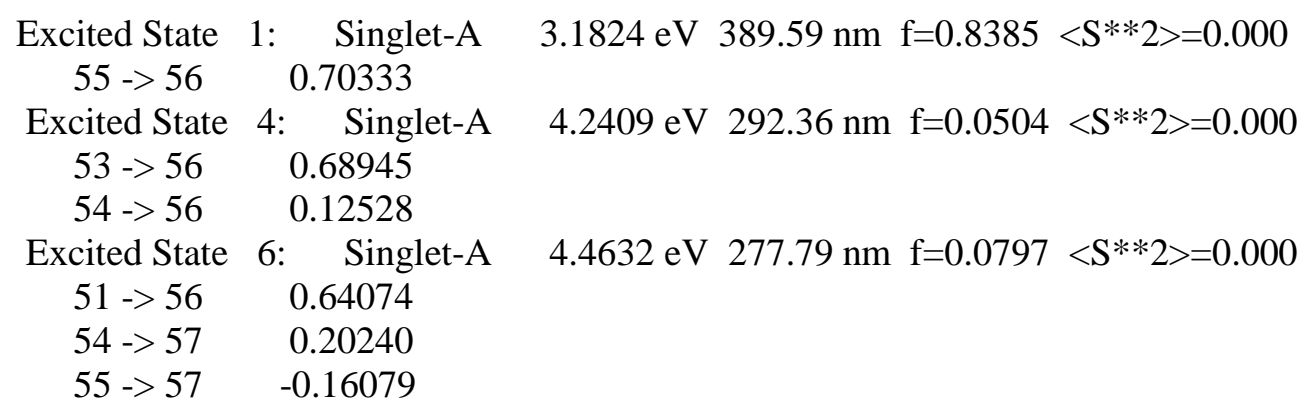

Table S5: TDDFT-predicted energies and expansion coefficients for compound 2 (only excited states with $\mathrm{f}>0.05$ and $\lambda>270 \mathrm{~nm}$ are listed).

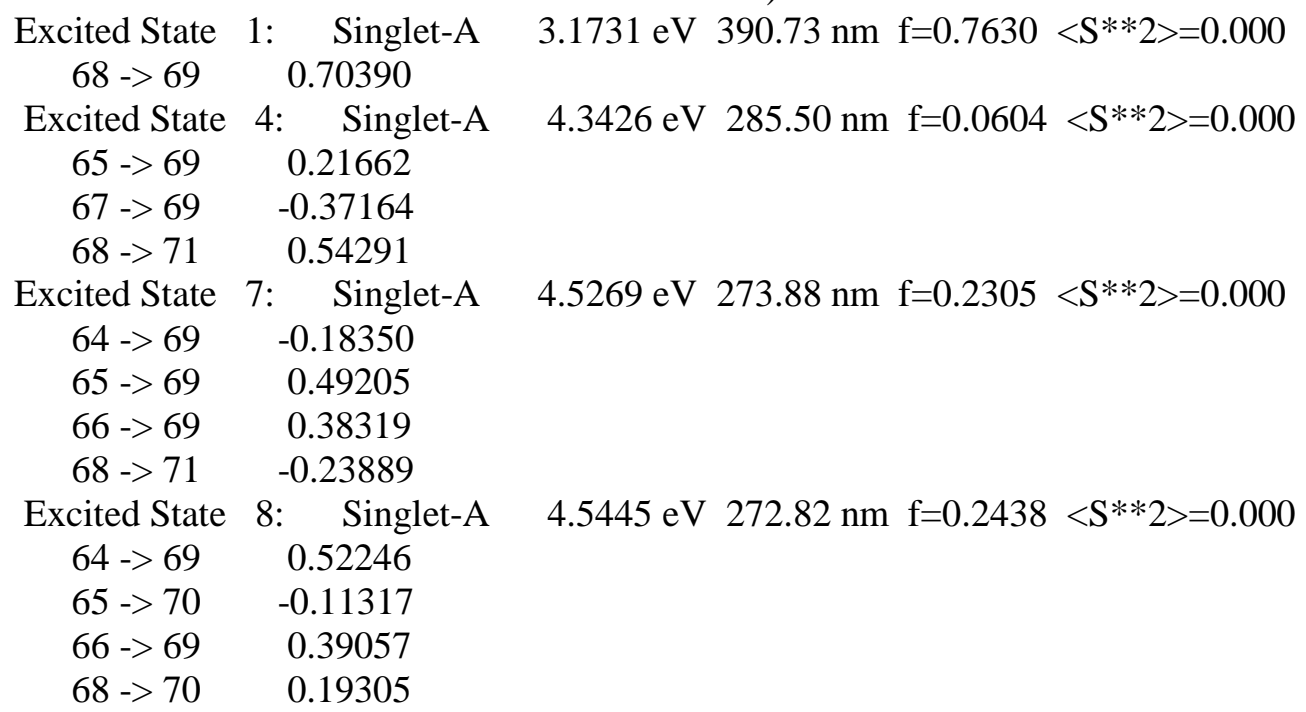

Table S6: TDDFT-predicted energies and expansion coefficients for compound $\mathbf{3}$ (only excited states with $\mathrm{f}>0.05$ and $\lambda>270 \mathrm{~nm}$ are listed).

Excited State 1: $\quad$ Singlet-A $\quad 3.6014 \mathrm{eV} \quad 344.26 \mathrm{~nm} \mathrm{f}=0.8120<\mathrm{S} * * 2\rangle=0.000$ 55 -> $56 \quad 0.70215$ 
Table S7: TDDFT-predicted energies and expansion coefficients for compound 4 (only excited states with $\mathrm{f}>0.05$ and $\lambda>270 \mathrm{~nm}$ are listed).

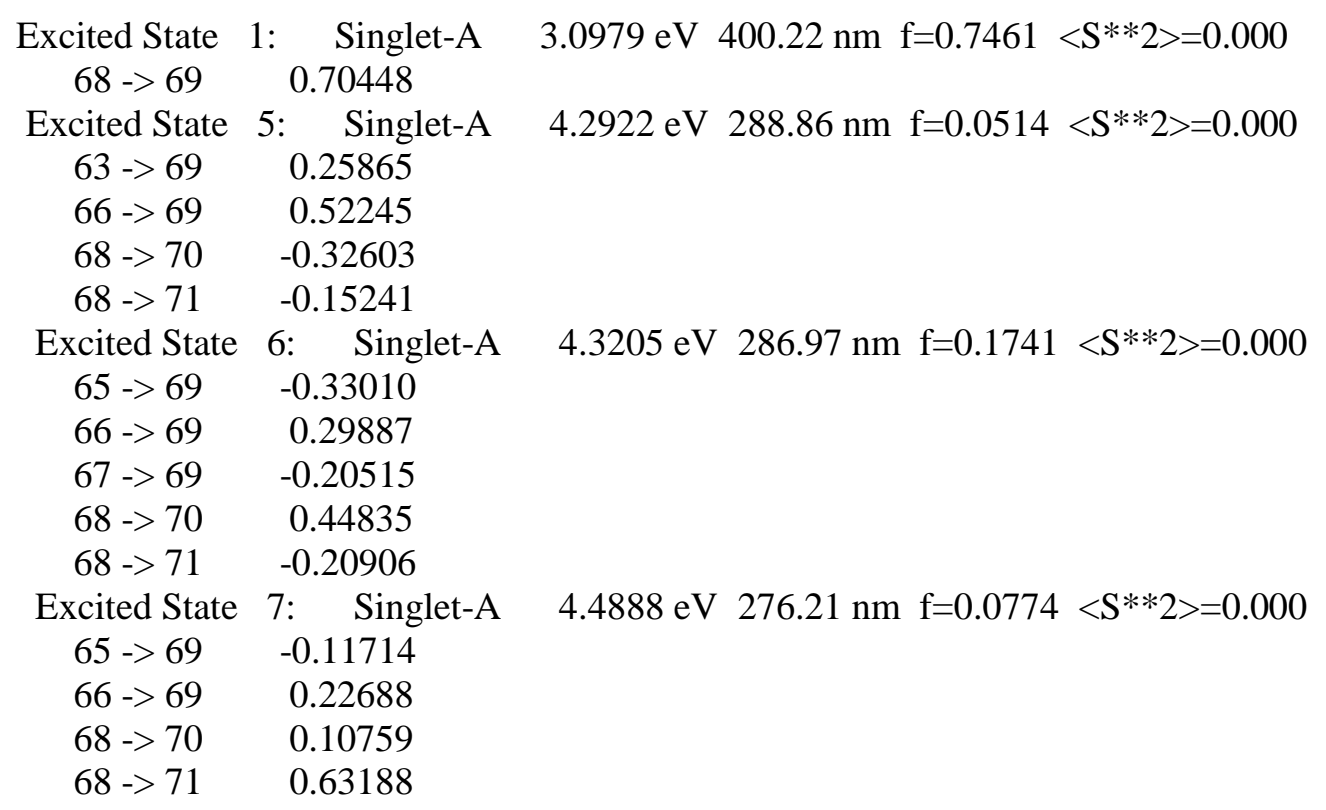

Table S8: TDDFT-predicted energies and expansion coefficients for compound $\mathbf{1 B F}_{2}$ (only excited states with $\mathrm{f}>0.05$ and $\lambda>270 \mathrm{~nm}$ are listed).

\begin{tabular}{|c|c|c|}
\hline $\begin{array}{c}\text { Excited State } \\
66 \text {-> } 67\end{array}$ & 1: Singlet-A & $2.9243 \mathrm{eV} 423.99 \mathrm{~nm} \mathrm{f}=0.6917$ \\
\hline Excited State & 6: $\quad$ Singlet-A & $4.3281 \mathrm{eV} \quad 286.46 \mathrm{~nm} \mathrm{f}=0.1988$ \\
\hline $62->67$ & 0.51432 & \\
\hline $63->68$ & -0.10646 & \\
\hline $66->68$ & 0.45617 & \\
\hline
\end{tabular}


Table S9: TDDFT-predicted energies and expansion coefficients for compound $\mathbf{2 B F}_{\mathbf{2}}$ (only excited states with $\mathrm{f}>0.05$ and $\lambda>270 \mathrm{~nm}$ are listed).

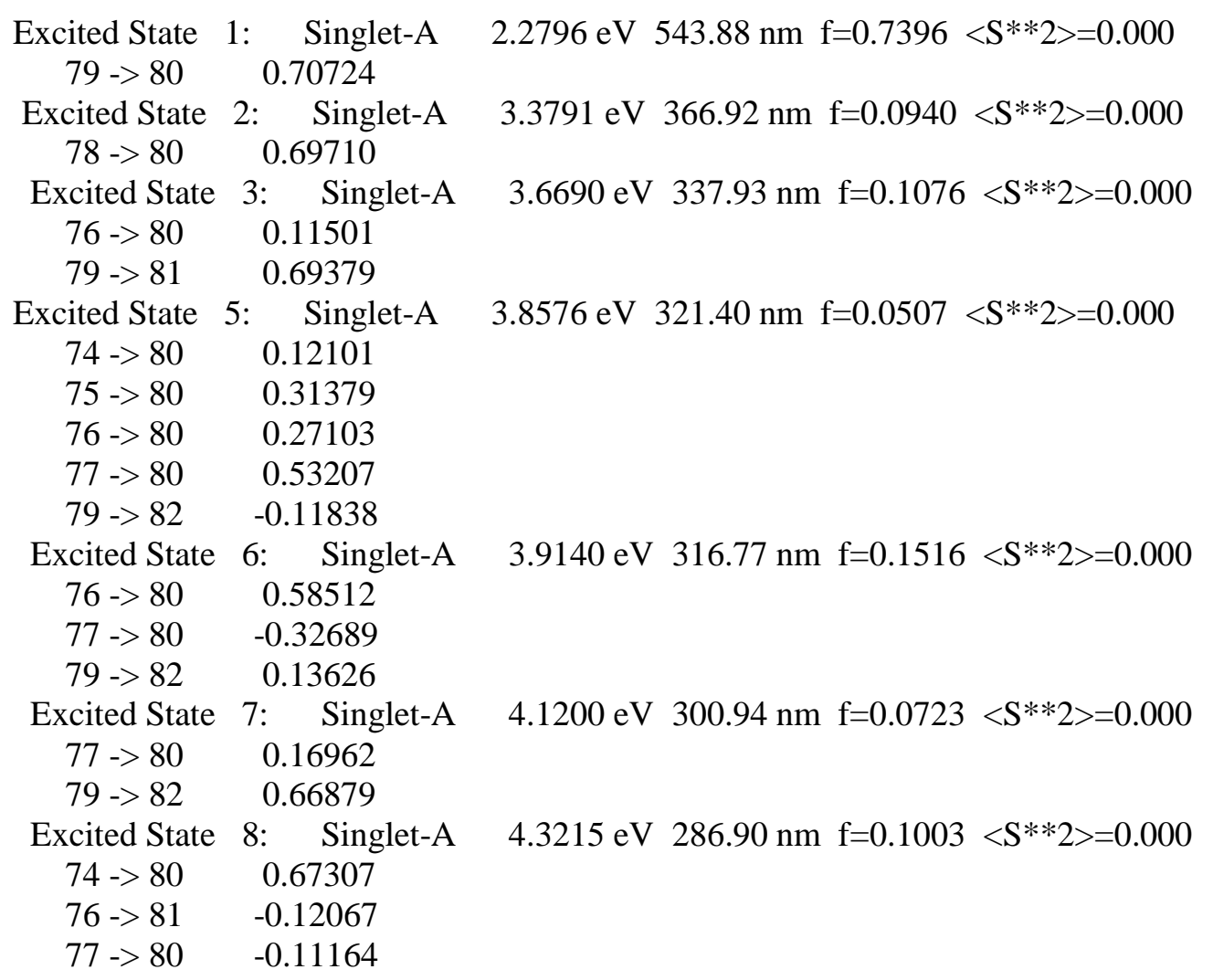

Table S10: TDDFT-predicted energies and expansion coefficients for compound $\mathbf{3 B F}_{2}$ (only excited states with $\mathrm{f}>0.05$ and $\lambda>270 \mathrm{~nm}$ are listed).

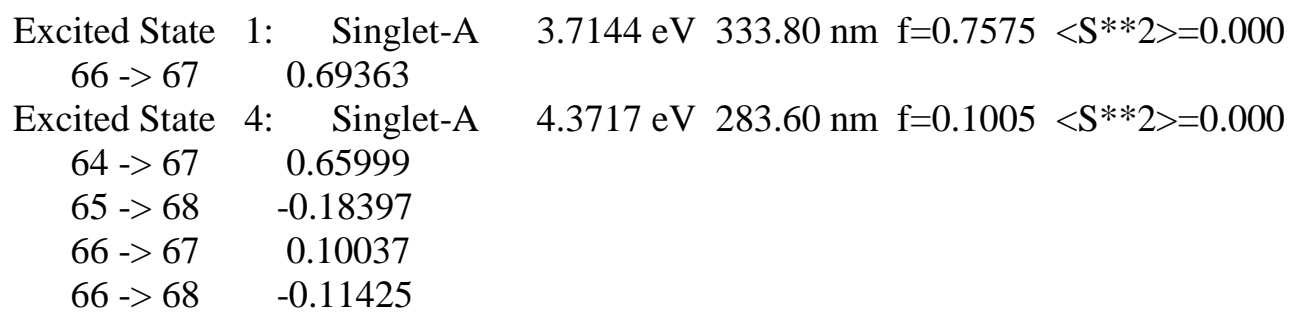


Table S11: TDDFT-predicted energies and expansion coefficients for compound $\mathbf{4 B F _ { 2 }}$ (only excited states with $\mathrm{f}>0.05$ and $\lambda>270 \mathrm{~nm}$ are listed).

\begin{tabular}{|c|c|c|}
\hline $\begin{array}{c}\text { Excited State } \\
79->80\end{array}$ & $\begin{array}{l}\text { 1: } \quad \text { Singlet-A } \\
0.70412\end{array}$ & $3.1556 \mathrm{eV} 392.90 \mathrm{~nm} \mathrm{f}=0.6719\langle\mathrm{~S} * * 2\rangle=0.000$ \\
\hline Excited State & 4: $\quad$ Singlet-A & $4.0991 \mathrm{eV} 302.46 \mathrm{~nm} \mathrm{f}=0.2371\langle\mathrm{~S} * * 2\rangle=0.000$ \\
\hline $76->80$ & 0.18200 & \\
\hline $77->80$ & 0.64000 & \\
\hline $78->80$ & 0.13572 & \\
\hline $79->81$ & -0.13147 & \\
\hline Excited State & 5: $\quad$ Singlet-A & $4.2016 \mathrm{eV} 295.09 \mathrm{~nm} \mathrm{f}=0.0780\langle\mathrm{~S} * * 2\rangle=0.000$ \\
\hline $76->80$ & 0.59888 & \\
\hline $77->80$ & -0.10310 & \\
\hline $78->80$ & -0.14571 & \\
\hline $79->81$ & 0.28328 & \\
\hline Excited State & 6: $\quad$ Singlet-A & $4.4351 \mathrm{eV} 279.55 \mathrm{~nm} \mathrm{f}=0.0769\langle\mathrm{~S} * * 2\rangle=0.000$ \\
\hline $74->80$ & -0.30362 & \\
\hline $76->80$ & -0.24036 & \\
\hline $77->80$ & 0.12398 & \\
\hline $78->80$ & 0.16832 & \\
\hline $79->81$ & 0.53977 & \\
\hline Excited State & 7: $\quad$ Singlet-A & $4.4997 \mathrm{eV} 275.54 \mathrm{~nm} \mathrm{f}=0.2223\left\langle\mathrm{~S}^{* *} 2\right\rangle=0.000$ \\
\hline $74->80$ & 0.59362 & \\
\hline $77->80$ & 0.14291 & \\
\hline $79->81$ & 0.29739 & \\
\hline $79->82$ & 0.10584 & \\
\hline
\end{tabular}


Table S12: B3LYP DFT excited state optimized geometry of compound $\mathbf{1 .}$

\begin{tabular}{|c|c|c|c|c|}
\hline \multirow{2}{*}{$\begin{array}{l}\text { Center } \\
\text { Number }\end{array}$} & Atomic & \multirow{2}{*}{$\begin{array}{l}\text { Atomic } \\
\text { Type }\end{array}$} & \multicolumn{2}{|c|}{ Coordinates (Angstroms) } \\
\hline & Numbe & & $\begin{array}{ll}X & Y\end{array}$ & $\mathrm{Z}$ \\
\hline 1 & 6 & 4.390398 & 0.583173 & 0.000325 \\
\hline 2 & 1 & 5.227284 & 1.268244 & 0.000648 \\
\hline 3 & 6 & 3.018476 & 0.882959 & 0.000363 \\
\hline 4 & 1 & 2.536008 & 1.843939 & 0.000736 \\
\hline 5 & 6 & 2.380945 & -0.362695 & -0.000070 \\
\hline 6 & 6 & 0.060322 & -0.073951 & -0.000332 \\
\hline 7 & 6 & -1.324372 & -0.611540 & -0.000340 \\
\hline 8 & 6 & -2.173042 & 0.500875 & -0.000121 \\
\hline 9 & 6 & -3.554364 & 0.358703 & 0.000366 \\
\hline 10 & 1 & -4.223940 & 1.212306 & 0.000634 \\
\hline 11 & 6 & -4.072549 & -0.941091 & 0.000448 \\
\hline 12 & 1 & -5.147005 & -1.087999 & 0.000775 \\
\hline 13 & 6 & -1.254976 & 1.664613 & -0.000184 \\
\hline 14 & 6 & -3.224746 & -2.053094 & 0.000148 \\
\hline 15 & 1 & -3.654449 & -3.049096 & 0.000238 \\
\hline 16 & 6 & -1.833853 & -1.900624 & -0.000219 \\
\hline 17 & 1 & -1.174693 & -2.761507 & -0.000362 \\
\hline 18 & 7 & -1.660557 & 2.933468 & 0.000213 \\
\hline 19 & 1 & -0.981183 & 3.678357 & -0.000740 \\
\hline 20 & 1 & -2.635023 & 3.184401 & -0.001483 \\
\hline 21 & 7 & 0.020809 & 1.316076 & -0.000334 \\
\hline 22 & 7 & 1.095083 & -0.846374 & -0.000306 \\
\hline 23 & 7 & 3.395030 & -1.281499 & 0.000039 \\
\hline 24 & 7 & 4.621108 & -0.734438 & 0.000084 \\
\hline 25 & 1 & 3.279257 & -2.283233 & -0.000619 \\
\hline
\end{tabular}


Table S13: B3LYP DFT excited state optimized geometry of compound 2.

\begin{tabular}{|c|c|c|c|c|}
\hline \multirow{2}{*}{$\begin{array}{l}\text { Center } \\
\text { Number }\end{array}$} & Atomic & \multirow{2}{*}{$\begin{array}{r}\text { Atomic } \\
\text { Type }\end{array}$} & \multicolumn{2}{|c|}{ Coordinates (Angstroms) } \\
\hline & Number & & X $\quad Y$ & Z \\
\hline 1 & 6 & 2.682492 & 1.675588 & 0.000051 \\
\hline 2 & 6 & 3.314556 & 0.330446 & 0.000038 \\
\hline 3 & 6 & 4.651041 & -0.048684 & -0.000114 \\
\hline 4 & 1 & 5.450356 & 0.685047 & -0.000196 \\
\hline 5 & 6 & 4.944901 & -1.413741 & -0.000236 \\
\hline 6 & 1 & 5.979618 & -1.738303 & -0.000429 \\
\hline 7 & 6 & 3.922383 & -2.370143 & -0.000145 \\
\hline 8 & 1 & 4.178890 & -3.423922 & -0.000186 \\
\hline 9 & 6 & 2.580393 & -1.987106 & 0.000004 \\
\hline 10 & 1 & 1.784965 & -2.723417 & 0.000100 \\
\hline 11 & 6 & 2.293332 & -0.627823 & 0.000072 \\
\hline 12 & 6 & 0.998595 & 0.078997 & 0.000207 \\
\hline 13 & 6 & -1.333676 & 0.221925 & 0.000048 \\
\hline 14 & 6 & -2.638992 & -0.378551 & -0.000061 \\
\hline 15 & 6 & -3.129860 & -1.693710 & 0.000119 \\
\hline 16 & 1 & -2.447081 & -2.536273 & 0.000175 \\
\hline 17 & 6 & -4.501022 & -1.880765 & 0.000187 \\
\hline 18 & 1 & -4.909387 & -2.885603 & 0.000305 \\
\hline 19 & 6 & -5.388080 & -0.777881 & 0.000075 \\
\hline 20 & 1 & -6.457210 & -0.962912 & 0.000160 \\
\hline 21 & 6 & -4.930063 & 0.528243 & -0.000164 \\
\hline 22 & 1 & -5.614161 & 1.369356 & -0.000245 \\
\hline 23 & 6 & -3.538426 & 0.711479 & -0.000211 \\
\hline 24 & 1 & 0.591935 & 2.141394 & -0.000155 \\
\hline 25 & 1 & -3.060663 & 2.798307 & 0.001332 \\
\hline 26 & 1 & 4.177245 & 2.833086 & 0.000064 \\
\hline 27 & 7 & 3.158443 & 2.858619 & 0.000128 \\
\hline 28 & 7 & 1.306953 & 1.425791 & 0.000396 \\
\hline 29 & 7 & -0.154617 & -0.496849 & 0.000107 \\
\hline 30 & 7 & -1.438613 & 1.552619 & 0.000034 \\
\hline 31 & 7 & -2.763586 & 1.836048 & -0.000686 \\
\hline
\end{tabular}

$\mathrm{E}_{h}=-852.179105$ Hartree 
Table S14: B3LYP DFT excited state optimized geometry of compound 3 .

\begin{tabular}{|c|c|c|c|c|}
\hline \multirow{2}{*}{$\begin{array}{l}\text { Center } \\
\text { Number }\end{array}$} & Atomic & \multirow{2}{*}{$\begin{array}{c}\text { Atomic } \\
\text { Type }\end{array}$} & \multicolumn{2}{|c|}{ Coordinates (Angstro } \\
\hline & Number & & $\begin{array}{ll}X & Y\end{array}$ & Y $\quad \mathrm{Z}$ \\
\hline 1 & 6 & -1.239922 & 1.747893 & -0.000382 \\
\hline 2 & 6 & -2.151178 & 0.569363 & -0.000147 \\
\hline 3 & 6 & -3.536990 & 0.510995 & 0.000010 \\
\hline 4 & 1 & -4.134993 & 1.415668 & -0.000055 \\
\hline 5 & 6 & -4.133061 & -0.752463 & 0.000215 \\
\hline 6 & 1 & -5.214158 & -0.836907 & 0.000367 \\
\hline 7 & 6 & -3.351412 & -1.914189 & 0.000271 \\
\hline 8 & 1 & -3.839418 & -2.882772 & 0.000404 \\
\hline 9 & 6 & -1.955636 & -1.848009 & 0.000102 \\
\hline 10 & 1 & 82 & -2.747078 & 0.000122 \\
\hline 11 & 6 & -1.369140 & -0.589637 & $-0.00005 c$ \\
\hline 12 & 6 & 0.0514 & -0.183942 & -0.000134 \\
\hline 13 & 6 & 2.363108 & -0.552354 & 0.000224 \\
\hline 14 & 6 & 3.4905 & -1.413285 & -0.000846 \\
\hline 15 & 1 & 3.484 & -2.491413 & -0.00177 \\
\hline 16 & 6 & 4.580448 & -0.571176 & -0.000512 \\
\hline 17 & 1 & 5.641920 & -0.762155 & -0.00108 \\
\hline 18 & 7 & 0.045148 & 1.209827 & -0.001171 \\
\hline 19 & 1 & 0.903 & 1.747394 & 0.000735 \\
\hline 20 & 7 & 1.046856 & -0.992185 & 0.000517 \\
\hline 21 & 7 & 2.747216 & 0.737269 & 0.001172 \\
\hline 22 & 7 & 4.089316 & 0.692331 & 0.000556 \\
\hline 23 & 1 & 4.613146 & 1.553498 & 0.001757 \\
\hline 24 & 8 & -1.511732 & 2.931737 & -0.000061 \\
\hline
\end{tabular}

$\mathrm{E}_{h}=-718.399972$ Hartree 
Table S15: B3LYP DFT excited state optimized geometry of compound 4.

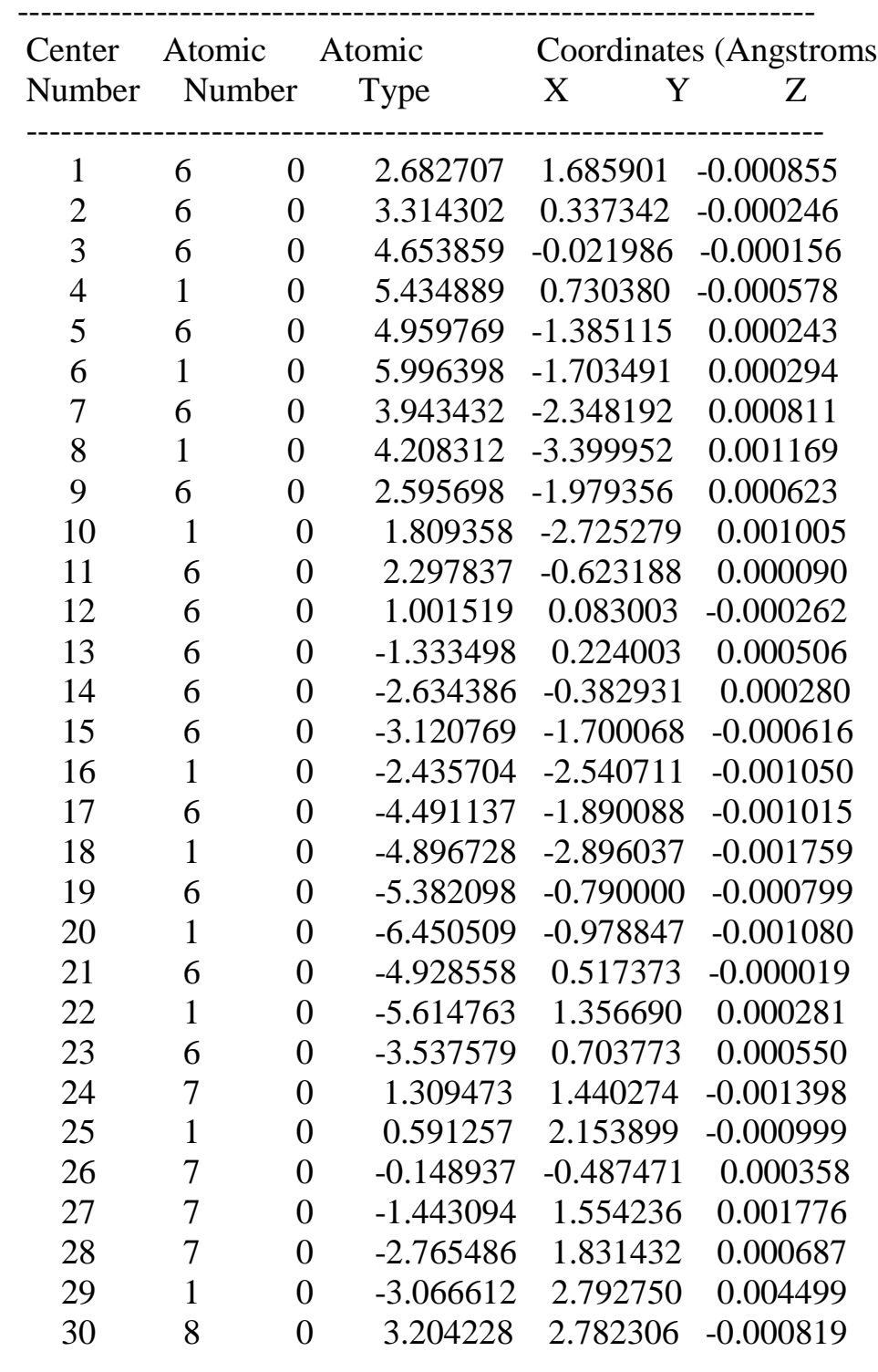

$E_{h}=-872.074950$ Hartree 
Table S16: B3LYP DFT excited state optimized geometry of compound $\mathbf{1 B F}_{2}$.

\begin{tabular}{|c|c|c|c|c|}
\hline \multirow{2}{*}{$\begin{array}{l}\text { Center } \\
\text { Number }\end{array}$} & Atomic & \multirow{2}{*}{$\begin{array}{r}\text { Atomic } \\
\text { Type }\end{array}$} & \multicolumn{2}{|c|}{ Coordinates (Angstrol } \\
\hline & Number & & X $\quad Y$ & $\mathrm{Z} \quad \mathrm{Z}$ \\
\hline 1 & 5 & 1.499435 & 1.168134 & 0.132762 \\
\hline 2 & 6 & -1.096696 & 1.344478 & -0.095606 \\
\hline 3 & 6 & -2.259172 & 0.453501 & -0.044953 \\
\hline 4 & 6 & -3.627652 & 0.698055 & -0.085122 \\
\hline 5 & 1 & -4.033602 & 1.700763 & \\
\hline 6 & 6 & -4.482047 & -0.403664 & -0.030753 \\
\hline 7 & 1 & -5.554333 & -0.248417 & -0.055036 \\
\hline 8 & 6 & -3.975114 & -1.706278 & 0.052303 \\
\hline 9 & 1 & -4.664729 & -2.541963 & 0.091102 \\
\hline 10 & 6 & 48 & -1.948 & 0.083833 \\
\hline 11 & 1 & 2.208501 & -2.957263 & 0.143218 \\
\hline 12 & 6 & -1.7 & -0.853091 & 0.038229 \\
\hline 13 & 6 & -0.289 & -0.753126 & 0.037557 \\
\hline 14 & 6 & 05 & -1.372471 & -0.030116 \\
\hline 15 & 6 & 0 & -2.208570 & -0.111103 \\
\hline 16 & 1 & 3.011540 & -3.287165 & -0.128825 \\
\hline 17 & 6 & 4.102760 & -1.325781 & -0.172286 \\
\hline 18 & 1 & 5.155792 & -1.559451 & -0.247082 \\
\hline 19 & 9 & 0 & 1.742534 & 1.400202 \\
\hline 20 & 9 & 1.716749 & 2.154221 & -0.842973 \\
\hline 21 & 7 & -1.108559 & 2.655216 & -0.212217 \\
\hline 22 & 1 & -0.244810 & 3.166521 & -0.334040 \\
\hline 23 & 1 & -1.972812 & 3.169939 & -0.276219 \\
\hline 24 & 7 & 0.033109 & 0.622288 & -0.015788 \\
\hline 25 & 7 & 0.567946 & -1.706597 & 0.038034 \\
\hline 26 & 7 & 2.364068 & -0.080183 & -0.048776 \\
\hline 27 & 7 & 3.712121 & -0.047793 & -0.128390 \\
\hline
\end{tabular}

$\mathrm{E}_{h}=-922.699332$ Hartree 
Table S17: B3LYP DFT excited state optimized geometry of compound $\mathbf{2 B F}_{2}$.

\begin{tabular}{|c|c|c|c|c|}
\hline \multirow{2}{*}{$\begin{array}{l}\text { Center } \\
\text { Number }\end{array}$} & Atomic & \multirow{2}{*}{$\begin{array}{l}\text { Atomic } \\
\text { Type }\end{array}$} & \multicolumn{2}{|c|}{ Coordinates (Angstroms } \\
\hline & Number & & $\begin{array}{ll}X & Y\end{array}$ & $\mathrm{Z}$ \\
\hline 1 & 5 & -0.113319 & 1.840444 & -0.155222 \\
\hline 2 & 6 & 2.393464 & 1.176013 & 0.134807 \\
\hline 3 & 6 & 3.207731 & -0.037993 & 0.078164 \\
\hline 4 & 6 & 4.581875 & -0.246396 & 0.146699 \\
\hline 5 & 1 & 5.284597 & 0.572657 & 0.248829 \\
\hline 6 & 6 & 5.040151 & -1.561390 & 0.074950 \\
\hline 7 & 1 & 6.104826 & -1.758730 & 0.119834 \\
\hline 8 & 6 & 4.144461 & -2.631532 & -0.052268 \\
\hline 9 & 1 & 4.531919 & -3.642826 & -0.103696 \\
\hline 10 & 6 & 2.766406 & -2.421360 & -0.112391 \\
\hline 11 & 1 & 2.075027 & -3.250290 & -0.206586 \\
\hline 12 & 6 & 2.308365 & -1.111520 & -0.049933 \\
\hline 13 & 6 & 0.961044 & -0.549046 & -0.071062 \\
\hline 14 & 6 & -1.314556 & -0.436168 & -0.054379 \\
\hline 15 & 6 & -2.653588 & -0.875194 & -0.000372 \\
\hline 16 & 6 & -3.301196 & -2.127458 & 0.002145 \\
\hline 17 & 1 & -2.731784 & -3.049330 & -0.050865 \\
\hline 18 & 6 & -4.677487 & -2.141795 & 0.072490 \\
\hline 19 & 1 & -5.206665 & -3.088952 & 0.074654 \\
\hline 20 & 6 & -5.430363 & -0.934220 & 0.143437 \\
\hline 21 & 1 & -6.512358 & -0.996707 & 0.197720 \\
\hline 22 & 6 & -4.820132 & 0.299068 & 0.144722 \\
\hline 23 & 1 & -5.392873 & 1.218588 & 0.199493 \\
\hline 24 & 6 & -3.406859 & 0.336904 & 0.070953 \\
\hline 25 & 9 & -0.040905 & 2.833119 & 0.830015 \\
\hline 26 & 9 & -0.064876 & 2.436266 & -1.418752 \\
\hline 27 & 7 & 2.821724 & 2.412141 & 0.286268 \\
\hline 28 & 1 & 2.166464 & 3.172182 & 0.408207 \\
\hline 29 & 1 & 3.804160 & 2.619374 & 0.375675 \\
\hline 30 & 7 & 1.092510 & 0.854421 & 0.017070 \\
\hline 31 & 7 & -0.163468 & -1.172026 & -0.111133 \\
\hline 32 & 7 & -1.350993 & 0.931944 & -0.013429 \\
\hline 33 & 7 & -2.599856 & 1.423501 & 0.056039 \\
\hline
\end{tabular}

$\mathrm{E}_{h}=-1076.370538$ Hartree 
Table S18: B3LYP DFT excited state optimized geometry of compound $\mathbf{3 B F}_{2}$.

\begin{tabular}{|c|c|c|c|c|}
\hline \multirow{3}{*}{$\begin{array}{l}\text { Center } \\
\text { Number } \\
1\end{array}$} & \multirow{2}{*}{$\begin{array}{l}\text { Atomic } \\
\text { Number }\end{array}$} & \multirow{2}{*}{$\begin{array}{l}\text { Atomic } \\
\text { Type }\end{array}$} & \multicolumn{2}{|c|}{ Coordinates (Angstroms } \\
\hline & & & $\begin{array}{ll}X & Y\end{array}$ & $\mathrm{Z}$ \\
\hline & 5 & 1.434391 & 1.236759 & 0.000120 \\
\hline 2 & 6 & 4.063272 & -1.416910 & -0.000170 \\
\hline 3 & 1 & 5.114954 & -1.653446 & -0.000227 \\
\hline 4 & 6 & 2.944820 & -2.227957 & 0.000009 \\
\hline 5 & 1 & 2.911863 & -3.304387 & 0.000051 \\
\hline 6 & 6 & 1.840877 & -1.354052 & 0.000013 \\
\hline 7 & 6 & -0.322689 & -0.668739 & -0.000002 \\
\hline 8 & 6 & -1.790399 & -0.792379 & -0.000011 \\
\hline 9 & 6 & -2.616504 & -1.907106 & 0.000049 \\
\hline 10 & 1 & -2.207563 & -2.910683 & 0.000091 \\
\hline 11 & 6 & -3.997042 & -1.685907 & 0.000026 \\
\hline 12 & 1 & -4.672981 & -2.533802 & 0.000055 \\
\hline 13 & 6 & -4.523490 & -0.389097 & -0.000028 \\
\hline 14 & 1 & -5.598956 & -0.251099 & -0.000037 \\
\hline 15 & 6 & -3.682731 & 0.728042 & -0.000059 \\
\hline 16 & 1 & -4.083763 & 1.735377 & -0.000096 \\
\hline 17 & 6 & -2.315686 & 0.500115 & -0.000082 \\
\hline 18 & 6 & -1.175697 & 1.459018 & -0.000129 \\
\hline 19 & 9 & 1.727562 & 1.979834 & 1.142408 \\
\hline 20 & 9 & 1.727834 & 1.980427 & -1.141678 \\
\hline 21 & 7 & -0.001158 & 0.675632 & -0.000117 \\
\hline 22 & 7 & 0.505640 & -1.664827 & 0.000100 \\
\hline 23 & 7 & 2.300496 & -0.080658 & -0.000157 \\
\hline 24 & 7 & 3.648990 & -0.136627 & -0.000173 \\
\hline 25 & 1 & 4.200057 & 0.708829 & -0.000678 \\
\hline 26 & 8 & -1.207537 & 2.669783 & -0.000198 \\
\hline
\end{tabular}

$\mathrm{E}_{h}=-942.587588$ Hartree 
Table S19: B3LYP DFT excited state optimized geometry of compound $\mathbf{4 B F} 2$.

\begin{tabular}{|c|c|c|c|c|}
\hline \multirow{2}{*}{$\begin{array}{l}\text { Center } \\
\text { Number }\end{array}$} & Atomic & \multirow{2}{*}{$\begin{array}{c}\text { Atomic } \\
\text { Type }\end{array}$} & \multicolumn{2}{|c|}{ Coordinates (Angstrol } \\
\hline & Number & & $X \quad Y$ & $Y \quad Z$ \\
\hline 1 & 5 & -0.045992 & 1.905833 & 0.004250 \\
\hline 2 & 6 & 2.497782 & 1.271405 & -0.003594 \\
\hline 3 & 6 & 3.264132 & -0.005518 & -0.002804 \\
\hline 4 & 6 & 4.630793 & -0.234282 & -0.005192 \\
\hline 5 & 1 & 5.337693 & 0.587777 & -0.008117 \\
\hline 6 & 6 & 5.062632 & -1.564303 & -0.003683 \\
\hline 7 & 1 & 6.124555 & -1.783321 & -0.005446 \\
\hline 8 & 6 & 4.143457 & -2.619479 & 0.000039 \\
\hline 9 & 1 & 4.507337 & -3.640920 & 0.001103 \\
\hline 10 & 6 & 2.765829 & -2.380257 & 0.002361 \\
\hline 11 & 1 & 2.053596 & -3.196957 & 0.005185 \\
\hline 12 & 6 & 2.346912 & -1.057509 & 0.000921 \\
\hline 13 & 6 & 1.000854 & -0.462695 & 0.002100 \\
\hline 14 & 6 & -1.264738 & -0.401405 & 0.002028 \\
\hline 15 & 6 & -2.607709 & -0.877748 & 0.001989 \\
\hline 16 & 6 & -3.190219 & -2.157239 & 0.003785 \\
\hline 17 & 1 & -2.568442 & -3.044674 & 0.007976 \\
\hline 18 & 6 & -4.567377 & -2.239365 & -0.000309 \\
\hline 19 & 1 & -5.051051 & -3.209373 & 0.000686 \\
\hline 20 & 6 & -5.369999 & -1.070804 & -0.006153 \\
\hline 21 & 1 & -6.449165 & -1.178180 & -0.009801 \\
\hline 22 & 6 & -4.824382 & 0.198287 & -0.007065 \\
\hline 23 & 1 & -5.445227 & 1.085868 & -0.011198 \\
\hline 24 & 6 & -3.422786 & 0.280112 & -0.002413 \\
\hline 25 & 9 & -0.083482 & 2.695576 & 1.150863 \\
\hline 26 & 9 & -0.084634 & 2.708129 & -1.133531 \\
\hline 27 & 7 & 1.130440 & 0.910141 & -0.000903 \\
\hline 28 & 7 & -0.111848 & -1.131768 & 0.004760 \\
\hline 29 & 7 & -1.297174 & 0.941994 & -0.001383 \\
\hline 30 & 7 & -2.591091 & 1.357959 & 0.001883 \\
\hline 31 & 1 & -2.811646 & 2.341459 & -0.032627 \\
\hline 32 & 8 & 2.917747 & 2.406540 & -0.005947 \\
\hline
\end{tabular}

$\mathrm{E}_{h}=-1096.2606847$ Hartree 


\section{References}

(1) Tamgho, I.-S.; Engle, J. T.; Ziegler, C. J. The Syntheses and Structures of Bis(Alkylimino)Isoindolines. Tetrahedron Lett. 2013, 54 (45), 6114-6117. https://doi.org/10.1016/j.tetlet.2013.08.134.

(2) Tkachuk, V.; Merkulova, V.; Omelchenko, I.; Arrault, A.; Hordiyenko, O. Cyclic Acyl Amidines as Unexpected C4-Donors for Fully Substituted Pyridine Ring Formation in the Base Mediated Reaction with Malononitrile. Tetrahedron Lett. 2019, 60 (30), 1959-1963. https://doi.org/https://doi.org/10.1016/j.tetlet.2019.06.038.

(3) Schrage, B. R.; Nemykin, V. N.; Ziegler, C. J. Biliazine: A Ring Open Phthalocyanine Analog with a Meso Hydrogen Bond. Chem. Commun. 2020, 56 (49), 6628-6631. https://doi.org/10.1039/D0CC03060K.

(4) Schrage, B. R.; Nemykin, V. N.; Ziegler, C. J. Subbiliazine: A Contracted Phthalocyanine Analog. Org. Lett. 2021, 23 (3), 1076-1080. https://doi.org/10.1021/acs.orglett.0c04291.

(5) Williams, A. T. R.; Winfield, S. A.; Miller, J. N. Relative Fluorescence Quantum Yields Using a Computer-Controlled Luminescence Spectrometer. Analyst 1983, 108 (1290), 1067-1071. https://doi.org/10.1039/AN9830801067.

(6) Sheldrick, G. M. A Short History of SHELX. Acta Crystallogr. Sect. A Found. Crystallogr. 2008, 64 (1), 112-122. https://doi.org/10.1107/S0108767307043930.

(7) Stephens, P. J.; Devlin, F. J.; Chabalowski, C. F.; Frisch, M. J. Ab Initio Calculation of Vibrational Absorption and Circular Dichroism Spectra Using Density Functional Force Fields. $J$. Phys. Chem. 1994, 98 (45), 11623-11627. https://doi.org/10.1021/j100096a001.

(8) Tomasi, J.; Mennucci, B.; Cammi, R. Quantum Mechanical Continuum Solvation Models. Chem. Rev. 2005, 105 (8), 2999-3093.

(9) McLean, A. D.; Chandler, G. S. Contracted Gaussian Basis Sets for Molecular Calculations. I. Second Row Atoms, Z = 11-18. J. Chem. Phys. 1980, 72 (10), 5639-5648.

https://doi.org/10.1063/1.438980.

(10) Frisch, M. J.; Trucks, G. W.; Schlegel, H. B.; Scuseria, G. E.; Robb, Ma.; Cheeseman, J. R.; Scalmani, G.; Barone, V.; Mennucci, B.; Petersson, G. A. Gaussian 09, Revision D. 01, Gaussian. Inc. Wallingford, CT 2009.

(11) Tenderholt, A. QMForge. Stanford University: Stanford, CA, USA. 